\title{
The genus Osbeckia (Melastomataceae) in India
}

\author{
Prashob P. ${ }^{1^{*}}$ \& Sibichen M. Thomas ${ }^{2}$ \\ ${ }^{1}$ CSIR-Central Insititute of Medicinal and Aromatic Plants Research Centre, Bengaluru, Karnataka - 560065 , India. \\ ${ }^{2}$ St. Joseph's College (Autonomous), Devagiri, Kozhikode, Kerala - 673 008, India. \\ *E-mail: prashobbotany@gmail.com
}

\begin{abstract}
The genus Osbeckia L. comprises about 50 species worldwide, is principally Asiatic in distribution. The genus is represented by 23 species and 4 varieties under four sections in India. The main centers of endemism are in Eastern Himalayas and the Western Ghats. A taxonomic treatment of Osbeckia in India is presented here with morphological descriptions, geographic distribution, notes, photo plates and identification key for sections, species and infra-specific taxa. Seven names are lectotypified and eight names are synonymised.
\end{abstract}

Keywords: Endemism, India, Lectotypification, Osbeckia, Taxonomy.

\section{Introduction}

The genus Osbeckia was established by Linnaeus (1753) in honour of Pehr Osbeck (1723-1805) who brought specimens of this plant to Sweden from China. Linnaeus named it Osbeckia chinensis L., the only species of this genus included in his Species Plantarum. Subsequently one more species, O. zeylanica L.f. was added to this genus from Sri Lanka by Carl Linné in Supplementum Plantarum (1781). Since then, a number of species were added to this genus from different parts of the world, mostly from Asia. Osbeckia is easily distinguished by its hypanthium covered with different types of hairs, bristles and emergences, isomorphic stamens, and ribbed capsules dehiscing apically by 4 or 5 pores.

Naudin (1850) recognized five groups, viz. Macrodesmiae, Antierotoma, Arrhinae, Oxyrhinae and Amphibolium. However, he did not indicate the

Received: 29.04.2019; Revised \& Accepted: 16.12.2019

Published Online: 31.12.2019 rank of these infrageneric groups. Triana (1871) in his treatment of Osbeckia included 32 species under 4 sections viz. Amblyanthera, Genuinae, Ceramicalyx and Asterostoma. Subsequently, Cogniaux (1891) in his monograph of Melastomataceae treated 51 species in six sections, viz. Asterostoma, Arrhinae, Genuinae, Ceramicalyx, Antherotoma and Pseudodissotis. C.B. Clarke (1879) in Flora of British India followed Triana (l.c.) and placed 26 species under 4 sections. However, Hansen (1977) did not follow any of these classifications in his revision of Asian Osbeckia and has opined that there is no necessity to define any subdivisions under this genus. The genus is currently placed under the tribe Melastomateae by Veranso-Libalah et al. (2017) based on phylogenetic data.

Hansen (1977) reported 17 species and 7 varieties from India. Since then, several taxa were described by various authors (Giri, 1983; Giri \& Nayar, 1983; Giri \& Nayar, 1984; Nayar \& Giri, 1984; Giri \& Nayar, 1986; Manickam \& Murugan, 2002; Kottaimuthu \& Gnanasekaran, 2015; Prashob et al., 2018) bringing the total number into 27 taxa. The present work is a comprehensive taxonomic treatment of the genus in India based on extensive field explorations and studying live and herbarium specimens in consultation with relevant types and protologue. The sectional classification of Triana (1871) is adopted with some modifications in this treatment. Twenty three species and four varieties under 4 sections are reported from India.

\section{Materials and Methods}

The study area comprises the entire political boundary of India which lies between $8^{\circ} 4^{\prime}$ and $37^{\circ} 6^{\prime}$ 
North latitudes and between $68^{\circ} 7^{\prime}$ and $97^{\circ} 25^{\prime}$ East longitudes. It comprises 28 states and 9 union territories. Specimens collected were processed for herbarium following Vogel (1987) and Bridson and Forman (1992). Plants were photographed with a Nikon D90 DSLR camera and photomicrographs using Leica Z4HD Stereo microscope attached with a Leica HD camera. The specimens were deposited at CALI, DEV and MH. The specimens from BLAT, BSI, CAL, CALI, DEV, FRLH, JCB, MGM, MH, SPU and SUK (acronyms as per Thiers, 2018) were studied. Photographs of herbarium sheets (types and authentic specimens) were obtained (or downloaded) from E, G, K, LINN, NY and W. The nomenclature was updated following Turland et al. (2018). All the species have been evaluated for their threat status using IUCN criteria $(2001,2019)$.

\section{Taxonomic treatment}

Osbeckia L., Sp. Pl. 345. 1753; L.f., Suppl. Pl. 215. 1782; D.Don, Prodr. Fl. Nepal. 221. 1825; A.DC., Prodr. 3: 142. 1828, Blume, Flora 14: 474. 1831; Naudin, Ann. Sci. Nat., Bot. ser. 3, 14: 56. 1850; Triana, Trans. Linn. Soc, London 28: 53. 1871; C.B.Clarke in Hook.f., Fl. Brit. India 2(6): 513. 1879; Cogn. in A.DC. \& C.DC., Monogr. Phan. 7: 311. 1891; C.Hansen, Ginkgoana 4: 84. 1977; Jie \& S.Renner., Fl. China 13: 361. 2007. Type: Osbeckia chinensis L.

Asterostoma Blume, Mus. Bot. 1(4): 50. 1849.

Ceramicalyx Blume, Mus. Bot. 1(4): 50. 1849.

Annual or perennial herbs or shrubs, erect, procumbent, much branched, rarely unbranched, 0.3-7.5 m tall. Young stems sub-quadrangular to quadrangular, very rarely winged at angles, covered with thin to dense hairs or bristles, hairs brown or purple, short to long, appressed to patent, straight or curved, rarely bulbous, sometimes shaggy, very rarely glabrous or hairy at nodes only. Leaves simple, opposite, petiolate, rarely sessile; petioles 1-15 mm long; lamina ovate to elliptic, or narrowly ovate to lanceolate, $1-15 \times 0.5-9 \mathrm{~cm}$; $3-5$ or $5-7-$ nerved, venation acrodromous, very rarely non acrodromous; apex acute, attenuate or very rarely acuminate; base obtuse to attenuate, subcordate, or sometimes cordate; margins entire, ciliate, glabrous on both surfaces or only above, or much more often with a thin or rarely dense indumentum of hairs, ascending or appressed or patent. Inflorescence terminal, consisting of few to many flowers, arranged in dense clusters in cymes or cymose, panicles, subtended by 1 or 2 pairs of leaves, occasionally solitary in upper leaf axils. Flowers actinomorphic, 4 or 5-merous; bracts ovate, triangular or orbicular, 1-17 mm long, margins ciliate, glabrous or with few to many appressed hairs; pedicels $0.5-5 \mathrm{~mm}$ long. Hypanthium urceolate, campanulate, obconic or globose, 2-14 × 1-7 mm, thinly to densely clothed with appressed to patent emergences or hairs or both. Intersepalar emergences $0.5-7 \mathrm{~mm}$ long, terete, glabrous or with few to many patent hairs, rarely appressed hairy, head stellate or with few hairs; other emergences similar, but smaller, size decreases towards base, restricted to the upper or lower part of the hypanthium; hairs patent, rarely reflexed, ascending or appressed, short to long, sometimes bristly, often curved, rarely bulbous. Sepals triangular, ovate, oblong or subulate, 1-14 × 1-7 $\mathrm{mm}$, apex obtuse, acute, rarely asymmetrical, often resembling the head of the stellate emergences, with few indistinct nerves, or with a single distinct, prominent nerve, glabrous dorsally, or with few to many, patent hairs mostly confined to nerve, caducous or persistent. Petals obovate, elliptic or orbicular, 5-29 × 2-18 mm, with various shades of purple, white, white tinged with pink, ciliate at least on apical part of margin, caducous. Stamens 8 or 10, equal; filaments $2.5-15 \mathrm{~mm}$ long; anthers narrowly ovate, 4-17 mm long including 2-7 mm long beak, yellow, twisted or not, pore 1 or 2 , on ventral side at the apex; connective widened into a variously shaped collar-like body surrounding the insertion of the filament immediately below the anther sacs. Ovary 4 or 5-celled, adnate to hypanthium for about half the length, deep anther pockets present or absent, crown present or absent; style 2-25 mm long, glabrous, stigma capitate. 
Capsules urceolate, campanulate, obconic or globose, 3-19 × 2-10 mm. Seeds minute, numerous, curved, 0.1-0.5 mm long.

Flowering \& fruiting: July-March.

Habitat: Occur in a wide variety of habitats such as evergreen forests, sholas, forest margins, mountains, grasslands as well as rock crevices and marshy swamps in higher elevations (c. $1000 \mathrm{~m}$ ).

Distribution: About 50 species (Mabberley, 2017), distributed in Africa, Australia, Bangladesh, Bhutan, China, India, Myanmar, Nepal, Sri Lanka and Thailand; 23 species and 4 varieties in India.

\section{Key to the sections of Osbeckia in India}

1a. Flowers tetramerous . .2

1b. Flowers pentamerous sect. Asterostoma 2a. Anthers not beaked sect. Amblyanthera

2b. Anthers beaked 3

3a. Herbs; hypanthium campanulate, neck absent sect. Osbeckia

3b. Shrubs; hypanthium urceolate, neck present sect. Ceramicalyx

\section{Osbeckia sect. Amblyanthera}

The section Amblyanthera was originally established by Triana (1871) to include three species viz., O. truncata, O. brachystemon and O. parvifolia. The section is characterized by tetramerous flowers, anthers without a beak and campanulate or obconic hypanthium with emergences and hairs. Clarke (1879) included four species namely, O. truncata, O. cupularis, O. parvifolia and O. rheedii, of which, the

Table 1. Sectional placement of Osbeckia in India

\begin{tabular}{|c|c|}
\hline Section & Name of taxa \\
\hline \multirow[t]{4}{*}{ Amblyanthera } & Osbeckia brachystemon Naudin \\
\hline & Osbeckia muralis Naudin \\
\hline & Osbeckia saddlepeakensis Prashob, Manudev, Sibichen \& Nampy \\
\hline & Osbeckia parvifolia Arn. \\
\hline \multirow[t]{14}{*}{ Asterostoma } & Osbeckia abrahamii G.S.Giri \& M.P.Nayar \\
\hline & Osbeckia aspera (L.)Blume \\
\hline & Osbeckia gracilis Bedd. \\
\hline & Osbeckia leschenaultiana DC. \\
\hline & Osbeckia mehrana G.S.Giri \& M.P.Nayar \\
\hline & Osbeckia nepalensis Hook. \\
\hline & Osbeckia nepalensis Hook. var. albiflora Lindl. \\
\hline & Osbeckia nutans Wall. ex C.B.Clarke \\
\hline & Osbeckia reticulata Bedd. \\
\hline & Osbeckia tirunelvelica Murugan \& Manickam ex Kottaim. \& Gnanasek. \\
\hline & Osbeckia travancorica Bedd. ex Gamble \\
\hline & Osbeckia virgata D.Don ex Wight \& Arn. \\
\hline & Osbeckia wightiana (Benth.) Wight \& Arn. \\
\hline & Osbeckia wynaadensis C.B.Clarke \\
\hline \multirow[t]{5}{*}{ Ceramicalyx } & Osbeckia nayarii G.S.Giri \\
\hline & Osbeckia rostrata D.Don \\
\hline & Osbeckia stellata Buch.-Ham. ex D.Don \\
\hline & Osbeckia stellata Buch.-Ham. ex D.Don var. hispidissima (Wight) C.Hansen \\
\hline & Osbeckia stellata Buch.-Ham. ex D.Don var. crinita (Benth. ex Naudin) C.Hansen \\
\hline \multirow[t]{4}{*}{ Osbeckia } & Osbeckia capitata Benth. \\
\hline & Osbeckia chinensis L. \\
\hline & Osbeckia chinensis L. var. pusilla (Zoll.) Triana \\
\hline & Osbeckia zeylanica L.f. \\
\hline
\end{tabular}


latter is a native of Sri Lanka. In India, this section consists of four species including the new taxa described recently (Prashob et al., 2018).

Osbeckia sect. Amblyanthera Triana, Trans. Linn. Soc., London 28: 53. 1871; C.B.Clarke in Hook.f., Fl. Brit. India 2(6): 514. 1879. Type: Osbeckia truncata D.Don (= O. muralis Naudin)

Arrhinae Naudin, Ann. Sci. Nat. Bot. ser. 3. 14: 56. 1850; Cogn. in A.DC. \& C.DC., Monogr. Phan. 7: 313. 1891.

Annual herbs; flowers tetramerous; anthers nonbeaked; hypanthium campanulate or obconic with emergences and hairs.

Distribution: Along wet tropical and subtropical Asia including India and Sri Lanka.

\section{Key to the species of sect. Amblyanthera}

1a. Anthers truncate at apex 2

1b. Anthers acute or obtuse at apex 3

2a. Petals purple; bracts ciliate O. muralis

2b. Petals white tinged with pink; bracts densely hairy O. brachystemon

3a. Hypanthium obconic with emergences only on the lower half; sepals elliptic O. saddlepeakensis

3b. Hypanthium globose with emergences throughout; sepals triangular ..... O. parvifolia

Osbeckia brachystemon Naudin, Ann. Sci. Nat. Bot. ser. 3, 14: 57. 1850; C.Hansen, Ginkgoana 4: 100. 1977; Manilal, Fl. Silent Valley 111. 1988; Subram., Fl. Thenmala Div. 141. 1995; Sasidh., Fl. Shenduruny W.L.S. 130. 1997; Sasidh., Fl. Periyar 144. 1998; Mohanan \& Sivad., Fl. Agasthyamala 278. 2002. Type: INDIA, Tamil Nadu, Nilgiris (as Nellighery), 1840, M. Perrottet 189 (holo P02441103 digital image!).

O. cupularis D.Don ex Wight \& Arn., Prodr. 323. 1834; C.B.Clarke in Hook.f., Fl. Brit. India 2: 514. 1879; Gamble, Fl. Pres. Madras 1: 494. 1919, p.p.

Fig. 1

Annual, erect, branched herbs, 10-65 cm tall, generally with profuse short branches at the base.
Stems covered with appressed, long, thin hairs. Petioles 1-4 mm long; lamina ovate, 2.2-4.5 × 1.5$2.5 \mathrm{~cm}$, apex acute to broadly acute or acuminate, base obtuse, margins ciliate, 3-5-nerved, both surfaces with patent, thin, long hairs. Inflorescence a terminal condensed panicle of 5-9 flowers, subtended by 1 or 2 pairs of leaves. Flowers tetramerous; bracts green, narrowly ovate, 1-2.5 × 0.5-1.5 mm long, densely hairy; pedicels $1-1.5 \mathrm{~mm}$ long. Hypanthium campanulate, 3-7 × 2-5 mm, thinly clothed with hairs and stellate emergences; intersepalar emergences stalked, 0.5-2 mm long, stalk with patent short hairs, head stellate with long bristles; other emergences a few, occurring only on the uppermost part of the hypanthium, hairs patent, light brown, long and thin. Sepals 4, persistent in fruit, triangular to attenuate, $1.2-3 \mathrm{~mm}$ long, 1-nerved, margins ciliate, tip stellate with long hairs, hairy on nerves. Petals 4 , obovate, 4-8 $\mathrm{mm}$ long, white tinged with pink, ciliate at apex. Stamens 8; filaments $2-3 \mathrm{~mm}$ long, white, glabrous; anthers oblong, 1-1.5 mm long, yellow, not twisted, not beaked, pores one or two, oblique on dorsal side; connective prolonged into a small collar. Ovary united with hypanthium for about half of its length; anther pockets absent; crown absent, clothed with bristles around base of style; style 3.5-5 mm long, glabrous. Capsules campanulate, slightly ribbed, 4-4.5 × 3-4 mm; seeds minute, $0.1-0.2 \mathrm{~mm}$ long, curved, muricate.

Flowering \& fruiting: August-November.

Chromosome number: $n=10$ (Meenakumari \& Kuriachan, 1990).

Habitat: Seen mostly on boarders of evergreen forests and grasslands at an elevation above $500 \mathrm{~m}$. This species occur as large clusters, usually in association with $O$. virgata and $O$. parvifolia.

Distribution: Endemic to South India.

Specimens examined: INDIA, Kerala, Idukki district, Anamudi Shola N.P., 08.11.14, Prashob \& Sibichen 5552 (DEV); Devikulam, 12.1910, A. Meebold 171757 (CAL); on the way to Edamalakudi, 23.08.2014, Prashob \& Sibichen 5530 


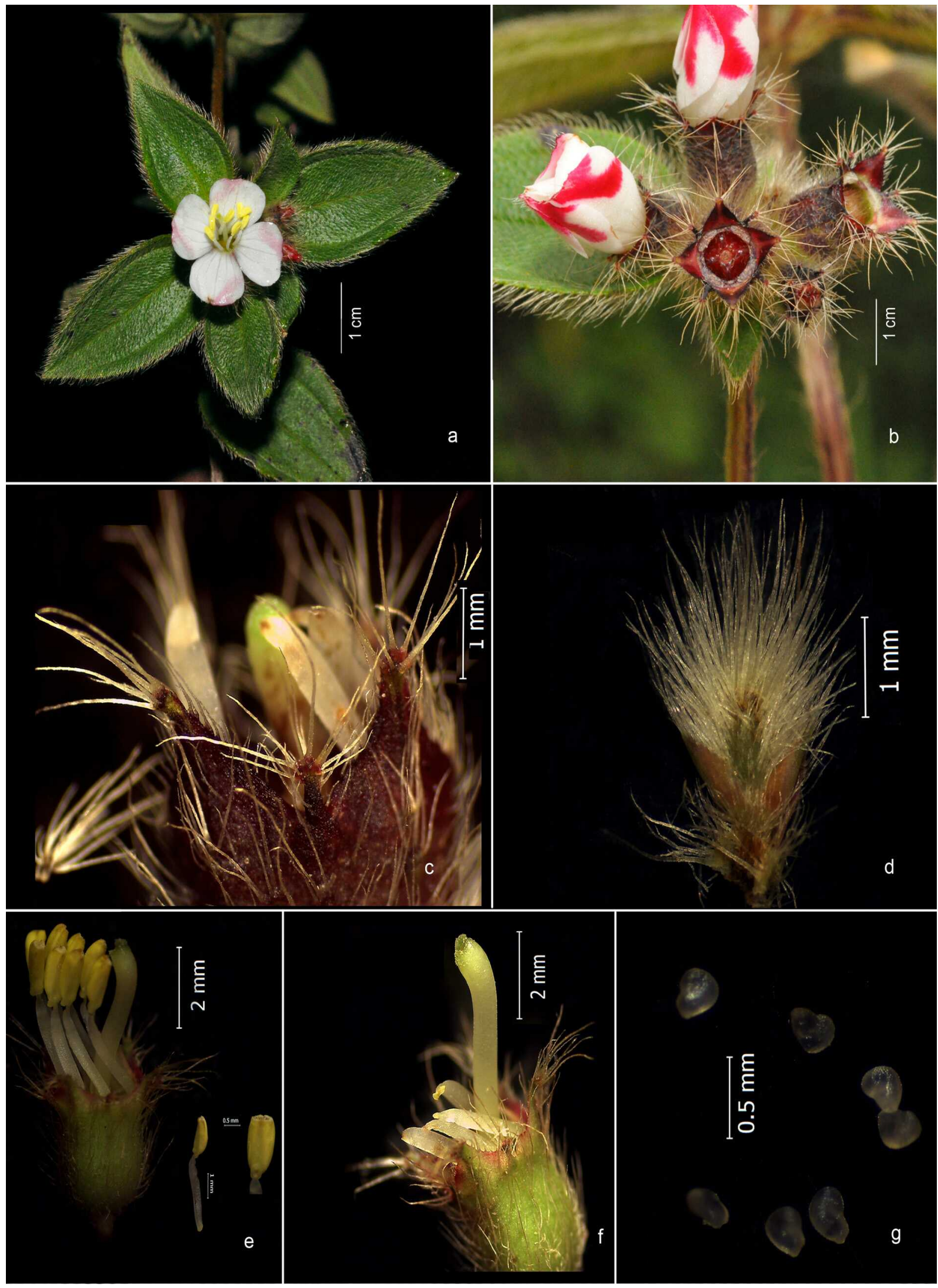

Fig. 1. Osbeckia brachystemon Naudin: a. Habit; b. Inflorescence; c. Calyx lobes with intersepalar emergences; d. Bract; e. Stamens and hypanthium; f. Gynoecium; g. Seeds. 
(DEV); Eravikulam N.P., Petimudi, 23.08.14, Prashob \& Sibichen 5528 (DEV); Methap, 08.11.14, Prashob E Sibichen 5555 (DEV); Munnar, Lockhurt gap, 10.10.1963, K.M. Sebastine 17507 (CAL); Palakkad district, Silent Valley, Anginda, 12.10.14, Prashob \& Sibichen 5539 (DEV); Sispara, 12.10.14, Prashob \& Sibichen 5537 (DEV); Vengitapal, 12.10.14, Prashob \& Sibichen 5540 (DEV). Tamil Nadu, Coimbatore district, s.loc., s.d., C.E.C.Fischer 171760 (CAL); Valparai, Anaimalais, 02.10.1984, K.M. Matthew 18314 (RHT); Dindigul district, Berijam slope, Kodaikanal, 06.08.1984, K.M. Matthew 40604 (RHT); Berijam Lake, Mathikettan range, 06.10.1994, V.S. Ramachandran 10333 (FRLH); Kodaikanal, 15.09.1956, J. Pallithanam 2135 (RHT); Leving path, Kodaikanal, 09.1955, K.M. Matthew 240 (RHT); Pillar rocks, Kodaikanal, 13.08.1912, S.J. Manju 171749 (CAL); Ibid., 27.07.1965, K.M. Matthew 24555 (RHT); Thoopithooki parai, 16.08.1987, K.M. Matthew 50254 (RHT); Upper Palani, 06.09.1911, C.E.C. Fischer 171756 (CAL); Vandaravu Marian shola, Kodaikanal, 29.08.1987, K.M. Matthew 50534 (RHT); Nilgiri district, Cinchona plantation, 24.02.19707, A.A. Ansari 1005 (CAL); Coonoor, 08.1883, J.S. Gamble 171743 (CAL); Kodanad, 10.11.1970, E. Vajravelu 3815 (MH); KotagiriAravenu, 22.10.1956, K. Subramanyam 1063 (CAL); Kunnacombai road, 01.09.1957, K.M. Sebastine 4205 (CAL); Mettukal, 28.07.1990, V.B. Hosagondan 162905 (MH); Morley lake, 20.07.1948, V. Narayanaswami 2232 (CAL); Mukuruti N.P., 20.09.2006, K. Ravikumar, R. Murugan \& Shyla 105175 (FRLH); Mullimund, 12.10.1972, K. Vivekananthan 42920 (MH); Naduvattam, 02.10.1956, N.P. Balakrishnan 141 (MH); Parsons valley, Ooty, 10.07.1970, J.E. Ellis 34607 (MH); way to Pykara, 12.09.1930, V. Narayanaswami 4216 (MH); Sholar, 26.08.1970, B.D. Sharma 35761 (MH); s.loc., 10.1910, A. Meebold 171755 (CAL); s.loc., 08.1887, G. King 171761 (CAL).

Notes: Osbeckia brachystemon is closely similar to O. parvifolia. They are usually found growing together at elevations above $500 \mathrm{~m}$. However, $\mathrm{O}$. brachystemon can be distinguished by its white flowers with pink blotches and 2-pored short anthers as opposed to pink to purple flowers and single pored, long anthers in O. parvifolia.

Osbeckia muralis Naudin, Ann. Sci. Nat. Bot. ser. 3, 14: 56. 1850; C.Hansen, Ginkgoana 4: 41. 1977; C.J.Saldanha, Fl. Karnataka. 42. 1984; Mohanan, Fl. Quilon Dist., 187. 1984; J.L.Ellis, Fl. Nallamalais 187. 1987; Ramach. \& V.J.Nair, Fl. Cannanore Dist. 187. 1988; Sasidh. et al., Bot. Stud. Med. Pl. Kerala 18. 1996; Sasidh. \& Sivaraj., Fl. Pl. Thrissur 190. 1996; Sivaraj. \& Mathew, Fl. Nilambur 273. 1997; Pull. \& Chennaiah, Fl. Andhra Pradesh 389. 1997; B.D.Sharma et al., Fl. Maharashtra, Dicot. 28. 2000; S.R.Yadav \& M.M.Sardesai, Fl. Kolhapur Dist., 196. 2002; Pull. et al., Fl. Eastern Ghats 56. 2002. K.G.Bhat, Fl. Udupi 219. 2003; G.V.S.Rao \& G.R.Kumari, Fl. Visakhapatnam Dist. Andhra Pradesh 339. 2003; Anil Kumar et al., Fl. Pathanamthitta 223. 2005; Sunil \& Sivad., Fl. Alappuzha Dist. 296. 2009. Lectotype (designated by Hansen, 1977): INDIA, Karnataka, Mangalore, R.F. Hohenacker 577 (P02441130 digital image!).

O. zeylanica Steud. ex Naudin., Ann. Sci. Nat. Bot. ser. 3, 14: 56. 1850.

O. truncata D.Don ex Wight \& Arn., Prodr. 332. 1834. C.B.Clarke in Hook.f., Fl. Brit. India 2: 514. 1879; Gamble, Fl. Pres. Madras 1: 494. 1919. O. truncata var. kurzii C.B.Clarke in Hook.f., Fl. Brit. India 2: 514. 1879. Type: INDIA, Bihar, Parasnath, s.d., Kurz s.n. (holo K000890021 digital image!)

O. truncata Arn., Companion Bot. Mag. 2: 308. 1837, nom. illeg.

Fig. 2

Annual, erect, branched, herbs, 5-55 cm tall. Stem quadrangular, sparsely covered with patent thin hairs, laxly villous. Petioles $2-9 \mathrm{~mm}$ long; lamina elliptic to ovate, $1-4.5 \times 0.8-2 \mathrm{~cm}$, apex acute, base obtuse, margins ciliate, both sides thinly clothed with ascending to thin patent hairs, 3-nerved. Inflorescence a terminal dense panicle of 6-15 flowers, subtended by 1 or 2 pairs of leaves, occasionally one or a few flowers arise from adjacent nodes. Flowers tetramerous; bracts 


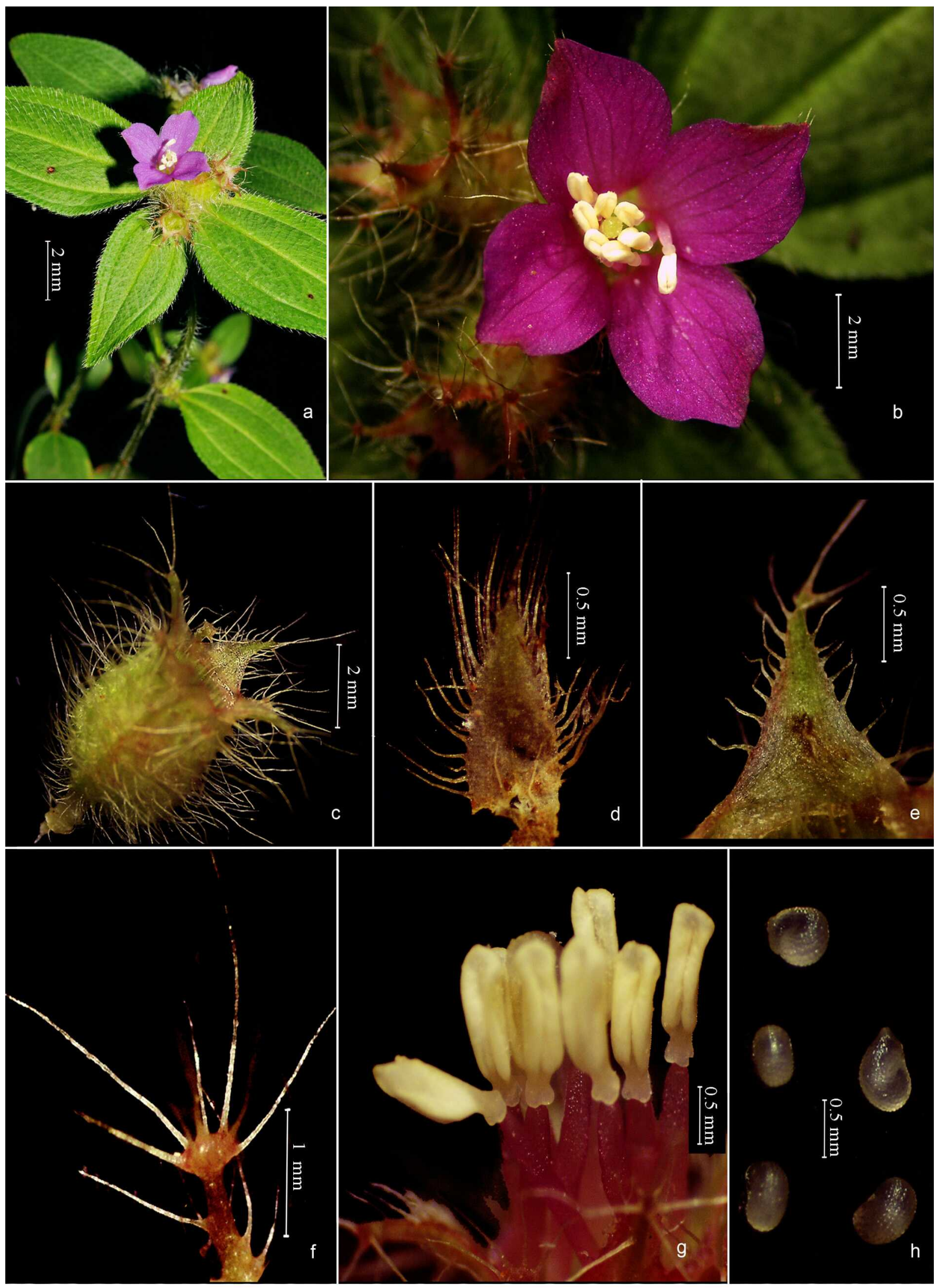

Fig. 2. Osbeckia muralis Naudin: a. Habit; b. Flower; c. Hypanthium; d. Bract; e. Calyx lobe; f. Intersepalar emergence; g. Stamens; h. Seeds. 
triangular, 1.5-3 mm long, margins ciliate; pedicels 1-3 $\mathrm{mm}$ long, covered with patent thin hairs. Hypanthium campanulate, 3-4.5 × 2-3.5 mm, sparsely covered with patent emergences and long hairs. Intersepalar emergence stalked, 0.5-1.5 mm long, stalk terete with patent hairs, head stellate with a few long persistent hairs. Other emergences similar, smaller in size towards base, or restricted to uppermost part of hypanthium. Sepals 4, broadly triangular, $0.5-2 \mathrm{~mm}$ long, 1-nerved, persistent in fruit, margins ciliate, apex with one to few patent hairs and sometimes emergences on nerves dorsally. Petals 4, broadly ovate, 4-6 × 3-4 mm, pink, with few hairs at apex. Stamens 8 , isomorphic; filaments 1-2 mm long; anthers ovate-oblong, 2-4 mm long, yellow, glabrous, apex truncate not twisted, pore large, oblique on dorsal side, connective prolonged into a small, slightly widened collar. Ovary united with hypanthium for about half its length, 1.5-2.5 mm long, 4-locular, anther pockets absent; crown absent, apically with many erect bristles; style 2-4 mm long, glabrous; stigma slightly widened. Capsules campanulate, ribbed, 3-4.5 × 2-3.5 mm; seeds numerous, $0.1-0.2 \mathrm{~mm}$ long, minute, curved.

Flowering \& fruiting: July-December.

Chromosome number: $n=10$ (Meenakumari \& Kuriachan, 1990).

Habitat: Occurs as clumps in lateritic soil and in rock crevices, between 50-650 m elevation. It appears on earth cuttings immediately after the monsoon.

Distribution: Endemic to India.

Specimens examined: INDIA, Karnataka, Mysore district, Hosuru Agumbe, 05.10.1962, R.S. Raghavan 83008 (CAL); Shimoga district, s.loc., 26.09.1978, K.P. Sreenath \& K.R. Keshava Moorthy 2910 (CAL). Kerala, Ernakulam district, Tripunithura, 10.10.1975, K.N. Subramanyam 5366 (CAL); Idukki district, Kattappana, 08.11.1981, R. Ramanujan \& C.N. Mohanan 72426 (CAL); Kozhikannam, 11.11.1975, K. Viveakananthan 46629 (CAL); Kannur district, Karimbam,
16.11.1977, s.coll. 52369 (CAL); Kollam district, Parackode, 11.08.1978, C.N. Mohanan 58464 (CAL); Kozhikode district, Medical College campus, 22.09.2014, Prashob \& Sibichen 5946 (DEV); Thondayad, 21.09.2014, Sibichen \& Prashob 5944 (DEV); Malappuram district, Calicut University campus, 17.07.1984, A. Babu 38296, (CAL); Palakkad district, Attapadi, South Malabar, 10.1910, C.E.C. Fischer 2305 (CAL); Kanjarampuzha, 26.10.1964, K.M. Sebastine 21074 (CAL); Pathanamthitta district, Punaloor, 24.08.1913, M. Ramaswami 1412 (CAL); Thrissur district, Chalakudi, 11.1910, A. Meebold 171884 (CAL); Edamadathara peak, 02.09.1913, C.C. Calder \& S. Ramaswami 497 (CAL); Travancore, 09.1884, J.S. Gamble 14694 (CAL). Maharashtra, Pune district, Ambavne village, 29.12.1964, s.coll. 101092 (CAL); Dajipur, s.d., Milind Sardesi s.n. (SUK); Dalkhand forest, 15.10.1967, K.V. Billore 111878 (CAL); Malani forest, 26.10.1967, K.V. Billore 113026 (CAL); Jamboti, s.d., Nilesh V. Mulpure 2381 (SUK). Uttarakhand, Hindugragh, 11.1891, Prain 171724 (CAL); s.loc., 12.1869, G. King 171717 (CAL). West Bengal, Jambalpur-Hatigirdha, 04.11.1986, S. Panda \& A.P. Das 270 (CAL).

Notes: Osbeckia muralis is one of the smallest species of the genus. It is often confused with O. chinensis var. pusilla, but can be distinguished by its thin patent hairs on the stem and much smaller flowers (4-8 mm diam.).

Osbeckia parvifolia Arn., Companion Bot. Mag. 2: 308. 1837; C.B.Clarke in Hook.f., Fl. Brit. India 2: 515. 1879; C.Hansen, Ginkgoana 4: 104. 1977; Ramach. \& V.J.Nair, Fl. Cannanore Dist. 188. 1988; B.D. Sharma et al., Fl. Maharashtra, Dicot. 28. 2000; S.R.Yadav \& M.M.Sardesai, Fl. Kolhapur Dist. 196. 2002. Amblyanthera parvifolia Blume, Mus. Bot. 1(4): 50. 1849. O. cupularis D.Don ex Wight \& Arn. var. parvifolia (Arn.) Trim., J. Ceylon Branch Roy. Asiat. Soc. 9: 34. 1885. Lectotype (designated by Hansen, 1977): SRI LANKA, s.d., Walker 332 (K000867959 digital image!).

O. cupularis D.Don ex Wight \& Arn., Prodr. Fl. Indi. Orient. 1: 323. 1834, p.p. 


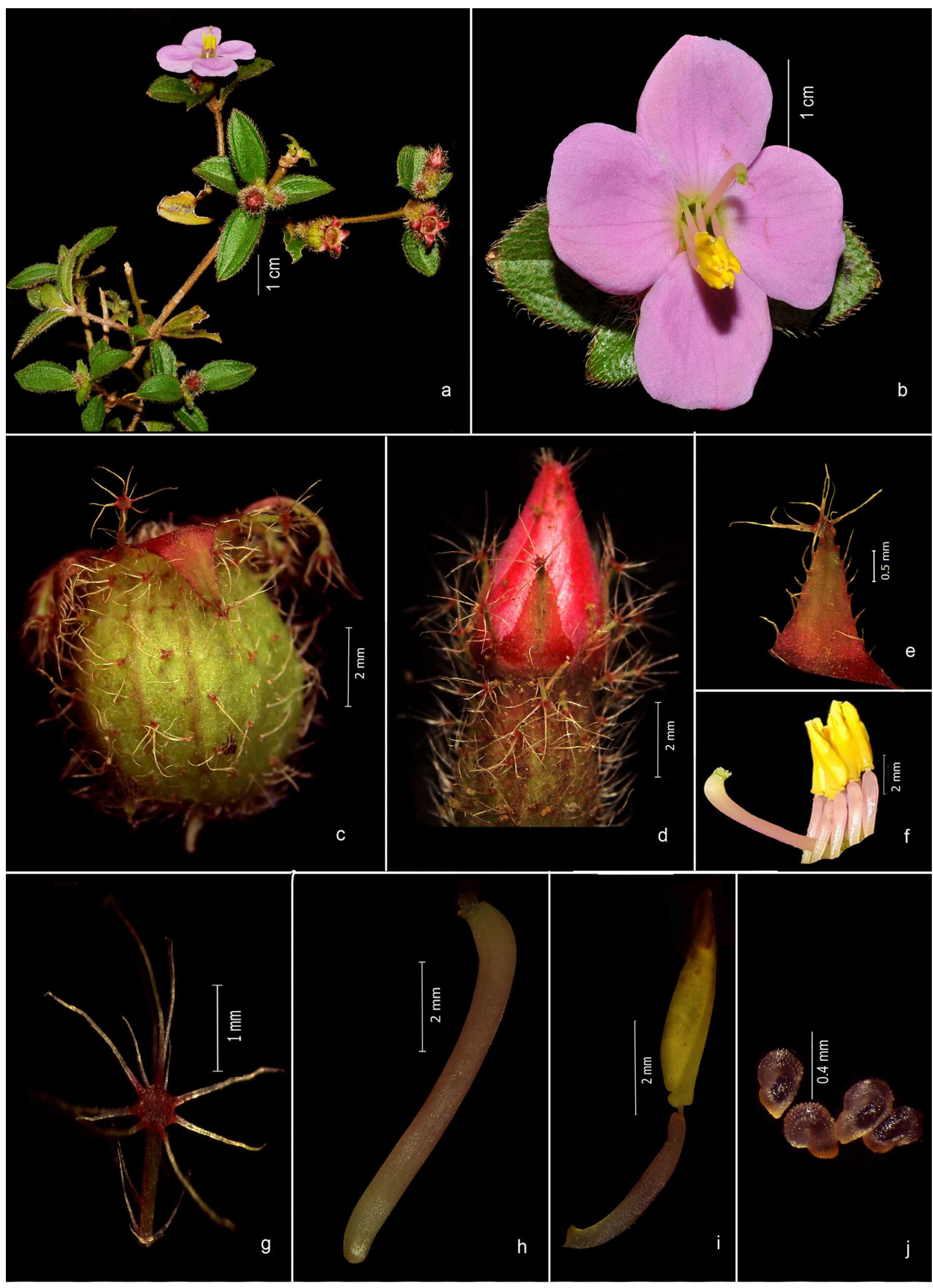

Fig. 3. Osbeckia parvifolia Arn.: a. Habit; b. Flower; c \& d. Hypanthium; e. Calyx lobe; f \& i. Stamens; g. Intersepalar emergence; h. Style; j. Seeds. 
O. confertiflora Naudin, Ann. Sci. Nat. Bot. ser. 3 14: 59. 1850. Type: INDIA, Tamil Nadu, Nellighery, s.d., Perrottet 235 (holo P02441140 digital image!).

O. erythrocephala Naudin, Ann. Sci. Nat. Bot. ser. 3, 14: 58. 1850. Osbeckia cupularis var. erythrocephala (Naudin) C.B.Clarke in Hook.f., Fl. Brit. India 2: 514. 1879. Type: SRI LANKA, 1838, Macrae s.n. (holo P02441138 digital image!).

O. cupularis var. purpurascens Wawra, Itin. Princ. S. Coburgi 1: 33. 1883. Type: SRI LANKA, Piselava, s.d., H. Wawra 1163 (holo W0056490 digital image!).

O. rosea Fyson, J. Indian Bot. Soc. 11: 49. 1932. Type: INDIA, Tamil Nadu, Nilgiri, s.d., Fyson 5731 (not traced).

Fig. 3

Prostrate to erect, branched herbs, c. 30-90 cm tall. Stems quadrangular, brownish, with appressed or patent to slightly retrorse, long hairs. Petioles 3-6 mm long; lamina green, ovate, $1.5-6 \times 0.8-3 \mathrm{~cm}$, 3 -nerved, apex acute to acuminate, base acute to broadly attenuate, both sides with ascending to patent, thin long hairs. Inflorescence a terminal loose cluster of 5-15 flowers, subtended by the upper 1 or 2 pairs of leaves; bracts ovate, $1.5-4 \times$ 0.5-1.5 mm, green, ciliate. Flowers tetramerous or pentamerous; pedicels 1-5 $\mathrm{mm}$ long. Hypanthium globose, 2-5 × 1.4-4 mm, clothed with emergences and hairs; intersepalar emergences 1-2 mm long, stalk slender, terete to slightly flattened, with a few patent hairs, head small, stellate with a few long purplish bristles, persistent; other emergences similar, patent, large on upper part and much reduced below; hairs patent, white to purplish. Sepals 4, triangular, $2-4 \times 0.5-2 \mathrm{~mm}$ long, one nerved, margins ciliate with a few patent hairs on nerve, persistent. Petals 4 , broadly obovate, 5$12 \times 4-10 \mathrm{~mm}$ long, pink to purple, ciliate at apex. Stamens 8, filaments 2-5 mm long; anthers narrowly ovate, $2-3.5 \mathrm{~mm}$ long, subobtuse at apex, pore slightly oblique on dorsal or ventral side, connective prolonged into a collar with a small dorsal lobe and a larger ventral lobe. Ovary united with hypanthium for about half of its length; anther pockets not extending to the base of ovary; crown ciliate; style 5-9 mm long. Capsules globose, 5-7 $\times 4.5-6.5 \mathrm{~mm}$; seeds minute, $0.1-0.2 \mathrm{~mm}$ long, numerous, curved.

Flowering \& fruiting: August-March.

Chromosome number: $n=10$ (Subramanyam, 1946)

Habitat: In forest margins, open grasslands and tea plantations at elevations between 600-1700 m.

Distribution: India and Sri Lanka.

Specimens examined: INDIA, Karnataka, Chikmagalur district, Bababudangiri, 03.03.2016, Prashob \& Sibichen 6023 (DEV); Kadur, 09.1893, W.A. Taecot 171759 (CAL); Kemmanaguddi, 05.09.1997, Ravikumar \& Udayan 09643 (FRLHT); Ibid., 29.09.1996, Devar 09463 (FRLHT); Ibid., 03.03.2016, Prashob \& Sibichen 6024 (DEV); Kutheramukha, 16.03.1997, Ramesh, Udayan \& Subramani 10220 (FRLHT); Tithkole, 19.10.1965, R.K. Arora 5080 (CAL). Kerala, Idukki district, Kadalar, Munnar, 09.1937, Barnes 119542 (DD); Marapalam, 20.01.1957 K.M. Sebastine 2109 (CAL); Rajamala, 02.02.1970, B.V. Shetty 31787 (CAL); Thiruvananthapuram district, Kallar, 09.1037, Barnes 119539 (DD); Wayanad district, Chembra, 08.12.2014, Prashob \& Sibichen 5559 (DEV); Tirunnelli, 16.11.1978, V.S. Ramachandran 17804 (CAL). Tamil Nadu, Coimbatore district, Sholayar submergible, 25.12.1963, K. Ramamurty 18097 (MH); Nilgiris district, Coonoor, 10.1910, Meebold 171754 (CAL); Ibid., s.d., J.S. Gamble 21380 (MH); Ibid., 18.11.1940, Brown 85986 (MH); Gudalur, 09.1937, Barnes 119540 (DD); Gudalur light house, 10.1910, A. Meebold 172075 (CAL); GudalurNaduvattam Road, 17.11.1958, K.M Sebastine 7313 (CAL, MH); Marapalam, Coonoor, 18.11.1940, Brown 85986 (MH); Ibid., 20.01.1957, K.M. Sebastine 2109 (MH); Ibid., Hulical Droog, 27.08.1957, K.M. Sebastine 4140 (CAL, MH); Kengarai, Kotagiri, 25.01.1972, E. Vajravelu 39636 (MH); Kotagiri Avenue, 22.10.1956, K. Subramanyam 1063 (MH); Naduvattam, 30.08.1970, B.D. Sharma 35929 (MH); Ooty, 09.1883, J.S. Gamble 12456 (DD); Nilgiri, 
10.1884, J.S. Gamble 172072 (CAL); Sholurmattam, 23.07.1970, E. Vajravelu 35058 (MH).

Notes: Osbeckia parvifolia closely resembles and often confused with O. brachystemon but can easily be distinguished by its globose hypanthium.

Osbeckia saddlepeakensis Prashob, Manudev, Sibichen \& Nampy, Phytotaxa 344 (2): 185-190. Type: INDIA, North Andaman Islands, Saddle Peak, 690 m, 29.09.2010, Santhosh Nampy \& K.M. Manudev 3526 (holo CAL; iso CALI, DEV, MH). Fig. 4

Erect, branched, annual herbs, $1.5-8 \mathrm{~cm}$ tall. Stems quadrangular, sparsely covered with patent thin hairs, laxly villous. Petioles 1-3 mm long; lamina ovate, $0.5-1.5 \times 0.3-1 \mathrm{~cm}$, apex acute, base obtuse, margins ciliate, both sides sparsely clothed with ascending to patent thin hairs, 3-nerved. Inflorescence a terminal short panicle, 3-5flowered, subtended by 1 or 2 pairs of leaves. Flowers tetramerous, 1-2 mm; pedicels $0.3-1.0 \mathrm{~mm}$ long, covered with thin hairs; bracts triangular, $0.3-0.8 \mathrm{~mm}$ long, ciliate. Hypanthium obconic, $1.8-2.5 \times 1.5-2.3 \mathrm{~mm}$, sparsely covered with emergences on lower portion, upper portion glabrous. Intersepalar emergences $0.5-1.0 \mathrm{~mm}$ long; head stellate with a few long persistent hairs; stalk terete, $0.2-0.5 \mathrm{~mm}$ long, glabrous. Other emergences sessile, smaller in size, restricted to lowermost part of hypanthium. Sepals 4, lanceolate, 1.5-2.5 mm long, ciliate, caducous. Petals 4, obovate, $1.5-3.0 \times 1-2 \mathrm{~mm}$, with few hairs at apex, pink. Stamens 8; filaments 2-2.5 mm long, pink; anthers ovate, 1-2 mm long, glabrous, acute at apex, not twisted; pore single, oblique on dorsal side, connective with conspicuous collar having two small dorsal lobes or tubercles and two ventral lobes. Ovary 4-locular, 1.8-1.5 mm long, united with hypanthium for about half its length; anther pockets present; crown absent; style 2-4 mm long, glabrous; stigma capitate. Capsules obconic, 4-ribbed, 1.8-2.5 × 1.5-2.3 mm, many seeded; seeds $0.2-0.5 \mathrm{~mm}$ long, minute, curved.

Flowering \& fruiting: August-November.
Habitat: Grows as a forest under growth at elevation between 650-700 $\mathrm{m}$.

Distribution: Endemic to India.

Notes: The species is named after the type locality 'Saddle Peak', the highest peak in Andaman and Nicobar Islands. Osbeckia saddlepeakensis is similar to O. muralis and O. brachystemon, but differs by its small flowers (2-4.5 mm across), triangular bracts, obconic hypanthium with emergences on basal portion only, short stalked intersepalar emergences, lanceolate and ciliate sepals and oblong to narrowly ovoid anthers with a single apical pore.

\section{Osbeckia sect. Asterostoma}

The section Asterostoma was established by Triana (1871) to include 19 species. The section is characterized by pentamerous flowers and campanulate hypanthium with emergences and hairs. Cogniaux (1891) followed the same sectional classification and accommodated 23 species. The members of this section are perennial shrubs except O. reticulata Bedd. which is a woody shrub. Clarke (1879) in Hooker's Flora of British India placed 14 species in this section. In the present treatment 13 species and one variety are recognized.

Osbeckia sect. Asterostoma Triana, Trans. Linn. Soc., London 28: 54. 1871; C.B.Clarke in Hook.f., Fl. Brit. India 2(6): 518. 1879; Cogn. in A.DC. \& C.DC., Monogr. Phan. 7: 313. 1891. Type: Asterostoma aspera Blume

Oxyrhinae Naudin, Ann. Sci. Nat. Bot. ser. 3, 14: 60. 1850.

Amphibolium Naudin, Ann. Sci. Nat. Bot. ser. 3, 14: 74.1850.

Perennial, erect, shrubs. Flowers pentamerous; hypanthium campanulate, either simple hairy or with emergences and hairs; anthers attenuate, straight, not beaked.

Distribution: Along wet tropical and subtropical Asia, including Bhutan, China, India, Nepal, and Sri Lanka. 


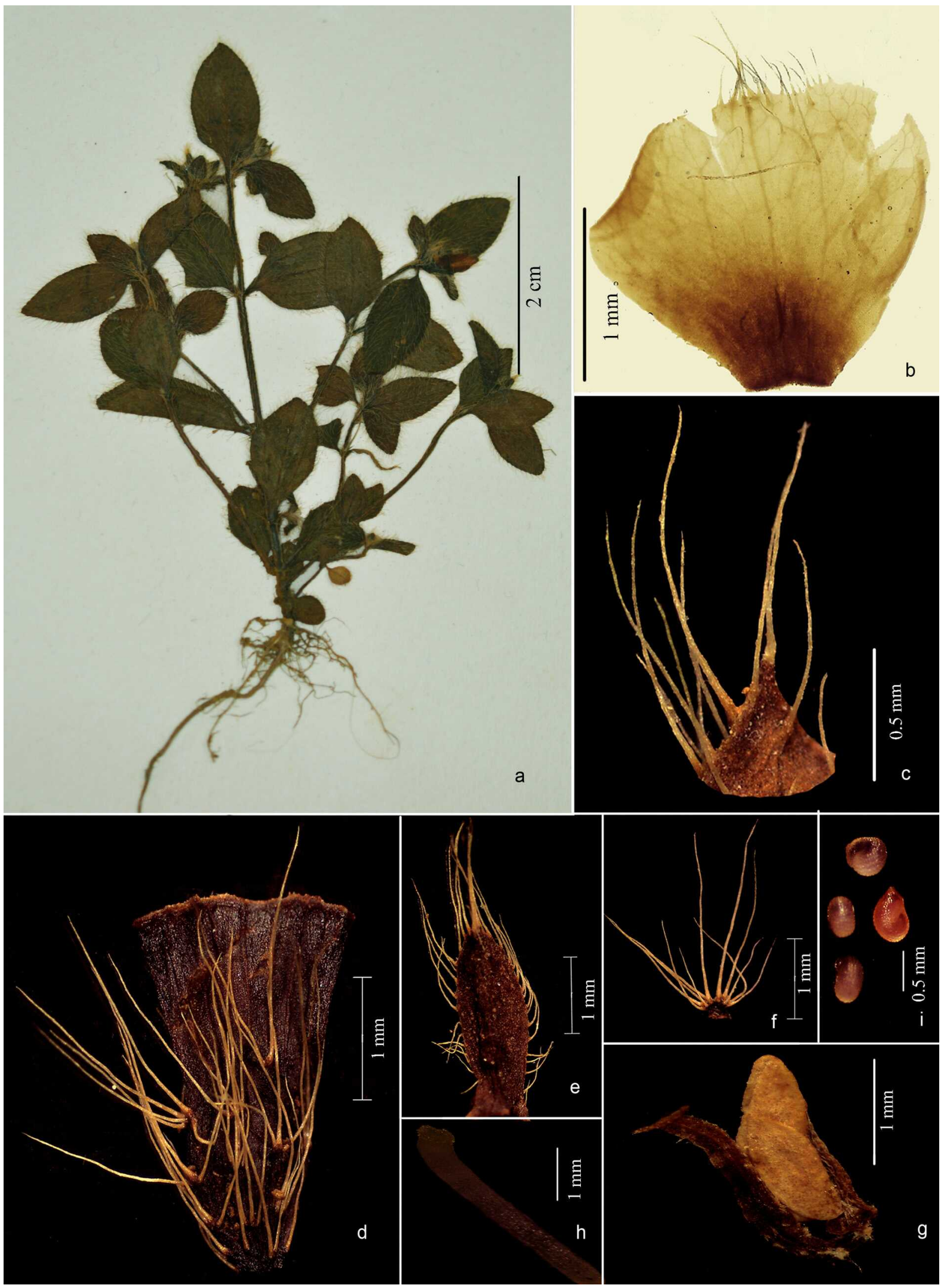

Fig. 4. Osbeckia saddlepeakensis Prashob, Manudev, Sibichen \& Nampy: a. Habit; b. Petal; c. Bract; d. Hypanthium; e. Calyx lobe; f. Intersepalar emergence; g. Stamen; h. Style; i. Seeds. 


\section{Key to the species of sect. Asterostoma}

1a. Hypanthium with simple hairs only 2

1b. Hypanthium with simple hairs, emergences and bristles ... 4

2a. Hairs (on hypanthium) with a bullate base, lamina turning yellow when dry ... O. gracilis

2b. Hairs (on hypanthium) without a bullate base, lamina not turning yellow when dry 3

3a. Sepals subulate; intersepalar emergences stalked, with terminal and lateral hairs

O. tirunelvelica

3b. Sepals triangular; intersepalar emergences sessile, with hairs at apex only O. aspera

4a. Hypanthium clothed with large scale-like emergences only 5

4b. Hypanthium clothed with stellate headed emergences 7

5a. Leaves $4 \times 1 \mathrm{~cm}$; panicle 2-3-flowered; sepals exceeding hypanthium O. nutans

5b. Leaves $8 \times 3 \mathrm{~cm}$; panicle $8-12$-flowered; sepals not exceeding hypanthium .. 6

6а. Lamina sessile, base truncate ....... O. nepalensis

6b. Lamina petiolate $(0.5-1.5 \mathrm{~cm}$ long), base obtuse O. wynaadensis

7a. Branchlets pilose; leaves sessile, ascending; bracts orbicular O. leschenaultiana

7b. Branchlets otherwise; leaves petiolate; bracts ovate to cordate 8

8a. Leaves 5-7-nerved 9

8b. Leaves 3-5-nerved 10

9a. Lamina bullate, densely hairy; base subcordate; venation acrodromous O. reticulata

9b. Lamina not bullate; sparsely hairy; base obtuse; venation non-acrodromous O. wightiana

10a. Inflorescence a terminal cluster of cymes; lamina lanceolate O. virgata
10b.Inflorescence a terminal panicle; lamina ovate to elliptic 11

11a. Bracts ovate, woolly dorsally; sepals triangular O. mehrana

11b.Bracts cordate, sparsely hairy; sepals subulate 12

12a. Calyx lobes symmetrical; branches sparsely hirsute O. travancorica

12b Calyx lobes asymmetrical; branches densely strigose O. abrahamii

Osbeckia abrahamii G.S.Giri \& M.P.Nayar, J. Bombay Nat. Hist. Soc. 81(2): 434. 1984. Type: INDIA, Kerala, Travancore, s.d., Narayanaswami 1379 (holo CAL0000015606!).

Fig. 5

Erect, perennial shrubs, 1-1.5 m tall. Stems quadrangular, branched, densely strigose. Petioles 5-15 mm long; lamina elliptic to lanceolate, green, 10-16 × 3-5 cm, 5-nerved, apex acute, base attenuate, both surfaces thinly clothed with short stiff sub-appressed to ascending hairs, hairs on the nerves longer. Inflorescence terminal or axillary lax panicles of 7-17 flowers. Flowers pentamerous; pedicels 1-3 $\mathrm{mm}$ long, covered with patent thin hairs; bracts ovate to cordate, $1.5-4 \times 2-6 \mathrm{~mm}$, green, broader than long, margins ciliate, appressed hairy above, glabrous beneath. Hypanthium campanulate, 4.5-7.5 × 3.5-6.5 mm, densely clothed with stellate or stalked emergences, intermixed with bristles and brown hairs; intersepalar emergences stalked, 1-3 mm long, stalk terete, with patent hairs, head stellate with tuft of hairs, caducous; other emergences smaller. Sepals 5 , subulate, asymmetric, $1.5-3.5 \times 2.5-4.5 \mathrm{~mm}$, broader than longer, with a prominent midrib having patent hairs dorsally; margins ciliate; apex with stellate emergences, caducous. Petals 5, obovate, $15-20 \times 11-14 \mathrm{~mm}$, pink. Stamens 10; filaments 5-8 $\mathrm{mm}$ long, glabrous; anthers narrowly ovate to oblong, 5.5-7 mm long, yellow, twisted, glabrous, pore apical large, oblique on dorsal side; connective prolonged in to a small, indistinctly lobed collar. Ovary 4-7 mm long, united with 


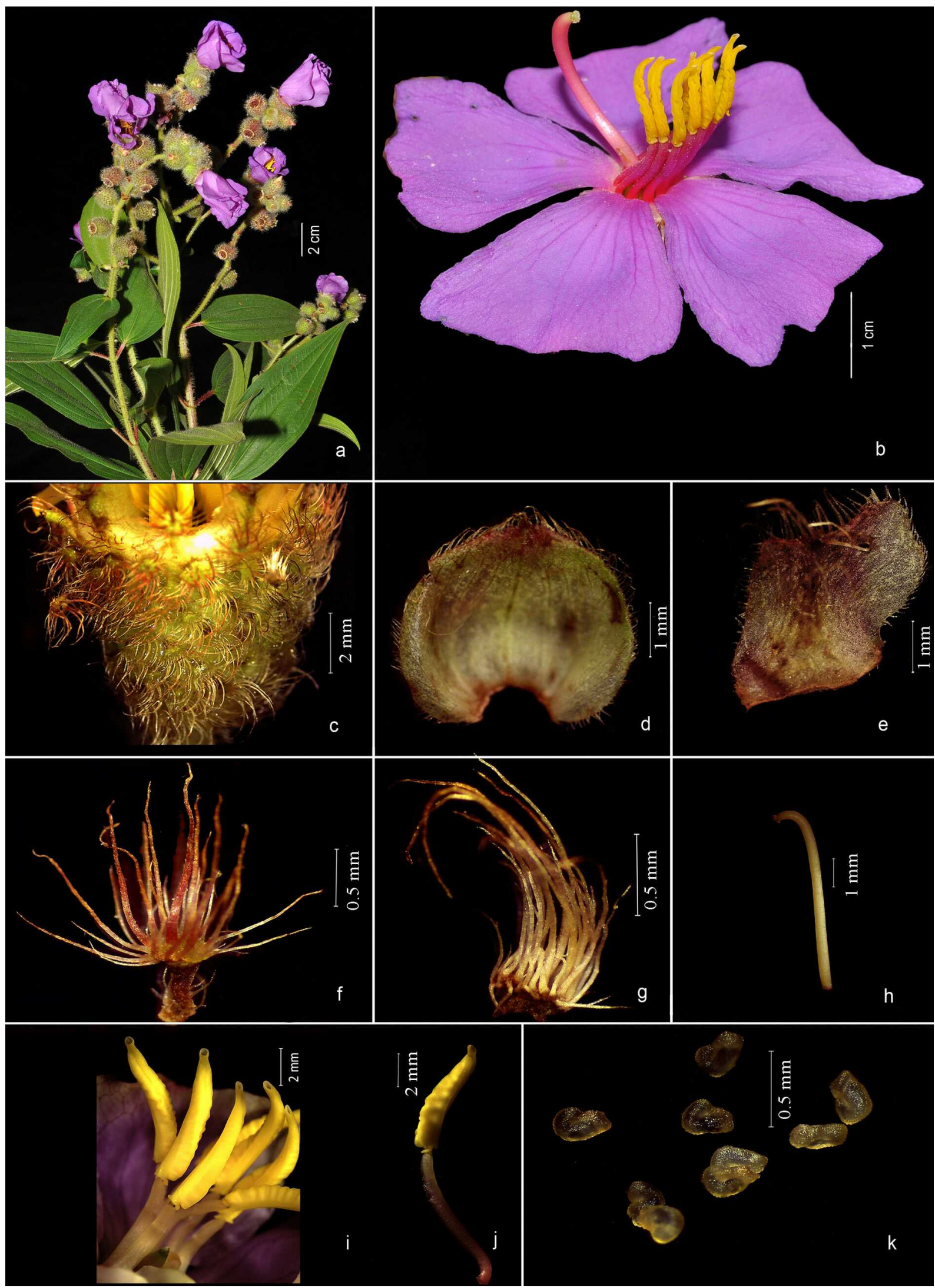

Fig. 5. Osbeckia abrahamii G.S.Giri \& M.P.Nayar: a. Habit; b. Flower; c. Hypanthium; d. Bract; e. Calyx lobe; f. Intersepalar emergence; g. Other emergences; h. Style; i \& j. Stamens; $\mathbf{k}$. Seeds. 
hypanthium; anther pockets covered with brownish appressed hairs; crown absent; style15-19 mm long, glabrous, curved; stigma slightly swollen. Capsules broadly urceolate, 7-9.5 × 4-5.5 mm; seeds numerous, minute, $0.1-0.2 \mathrm{~mm}$ long, curved.

Flowering \& fruiting: July-December.

Habitat: Found in forest borders and in open grasslands of elevation above $300 \mathrm{~m}$.

Distribution: Endemic to South India.

Specimens examined: INDIA, Kerala, Kottayam district, Vagamon, 20.11.2014, Prashob \& Sibichen 5564 (DEV); Pathanamthitta district, Ranni, 30.07.2014, Prashob \& S. Syam Radh 5510 (DEV).

Notes: It resembles O. travancorica but distinguished by its long, lax panicles and asymmetric sepals as opposed to the short, compact panicles and symmetric sepals in O. travancorica.

Osbeckia aspera (L.) Blume, Flora. 14: 474. 1831; C.B.Clarke in Hook.f., FI. Brit. India 2: 519. 1879; Gamble, Fl. Pres. Madras 1: 492. 1919. Melastoma aspera L., Sp. Pl. 391. 1753. Asterostoma aspera (L.) Blume, Mus. Bot. 1(4): 50. 1849. O. aspera (L.) Blume var. aspera, Ginkgoana 4: 82. 1977; Vivek. in N.C.Nair \& A.N.Henry, Fl. Tamil Nadu 1: 161. 1983; Mohanan, Fl. Quilon Dist., 186. 1984; Manilal, Fl. Silent Valley 110. 1988; Vajr., Fl. Palghat Dist., 203. 1990; M.Mohanan \& Henry, Fl. Thiruvanthapuram 198. 1994; Subram., Fl. Thenmala Div. 142. 1995; Sasidh. et al., Bot. Stud. Med. Pl. Kerala 24. 1996; Sasidh. \& Sivar., Fl. Pl. Thrissur For. 189. 1996; Sasidh., Fl. Periyar Tiger Reserve 143. 1998; Sasidh., Fl. Parambikulam W.L.S. 127. 2002; Mohanan \& Sivad., Fl. Agasthyamala 278. 2002; Anil Kumar et al., Fl. Pathanamthitta 222. 2005; Manickam et al., Fl. Tirunelveli Hills 406. 2008; Sunil \& Sivadasan, Fl. Alappuzha Dist., 296. 2009. O. aspera (L.) Blume var. typica C.B.Clarke in Hook.f., Fl. Brit. India 2: 519. 1879. Lectotype (designated by Hansen, 1977): SRI LANKA, s.d., Herman s.n. (BM000621501 digital image!).
O. kleinii Wight \& Arn., Prodr. Fl. Ind. Orient. 1: 323. 1834. Asterostoma kleinii (Wight \& Arn.) Blume, Mus. Bot. 1(4): 50. 1849. Osbeckia aspera Blume var. kleinii (Wight \& Arn.) C.B.Clarke in Hook.f., Fl. Brit. India 2: 519. 1879. Type: SRI LANKA, Trincomalee, 25.01.1796, Klein s.n. (holo K000867979 digital image!)

O. glauca Wall. ex Naudin, Ann. Sci. Nat., Bot. sér. 3, 14: 68. 1850

O. kewensis C.E.C.Fisch., Bull. Misc. Inform. Kew 1938(1): 34. 1938. Lectotype (designated here): SRI LANKA, s.loc., s.d., s.n. (K000867986 digital image!).

O. minor Triana, Trans. Linn. Soc. London 28(1): 55. 1872. Type: SRI LANKA, s.loc., s.d., Thwaites 1569 (holo K000867985 image!).

O. courtallensis Gamble, Bull. Misc. Inform. Kew 1918(7): 242. Type: INDIA, Tamil Nadu, Courtallum, Tirunelveli, Wight 1103 (holo K000867983 digital image!).

O. lawsonii Gamble, Bull. Misc. Inform. Kew 1918(7): 242. Type: INDIA, Travancore, $650 \mathrm{~m}$., 03.12.1893, M.A. Lawson 46 (holo K000867989 digital image!).

Melastoma glaucum Wall., Numer. List n. 4055. 1831, nom. nud.

Katou kadalai Rheede, Hortus Malabaricus. 43.91.1493, non. inval.

Fig. 6

Perennial, erect shrubs, 1-2.5 m tall. Stems branched, young stems quadrangular, sparsely clothed with appressed to patent hairs, intermixed with curved strong bristles. Petioles 5-10 mm long; lamina green, ovate to lanceolate, $2-11 \times 1-4.5$ $\mathrm{cm}, 3-5$-nerved, apex acute to acuminate, base acute, both sides thinly clothed with patent hairs. Inflorescence terminal, 6-12-flowered cymes or a panicle of 2 or 3 such cymes, subtended by bracts and a pair of leaves. Flowers pentamerous, pedicels 2-5 mm long; bracts green, ovate to cordate, 1-5 $\mathrm{mm}$ long, ciliate and densely appressed-hairy. Hypanthium campanulate, 4-8 × 3-6 mm, with a thin cover of hairs; intersepalar emergences 0.5-2.5 mm long, sessile, ending in a tuft of hairs; 

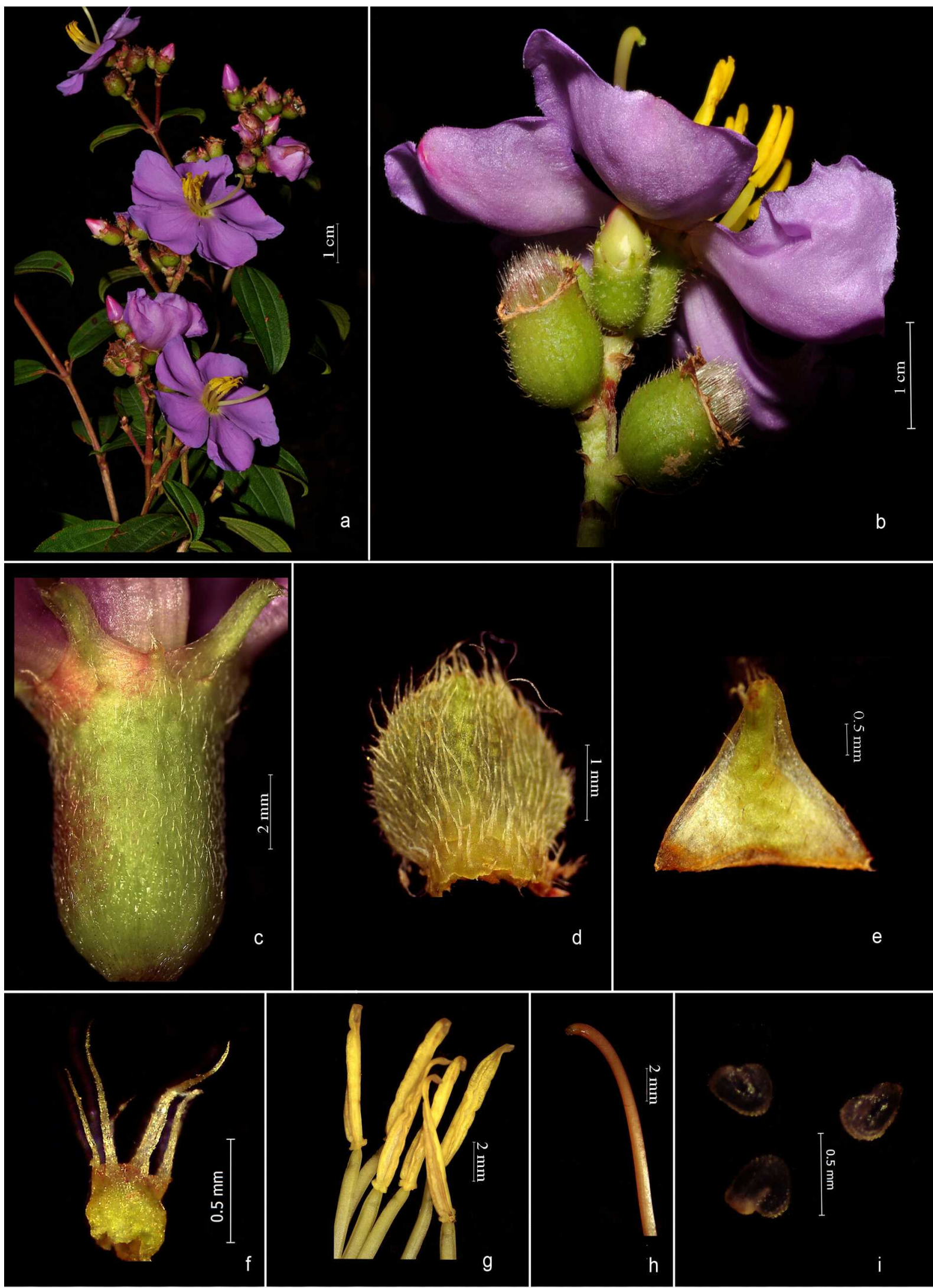

Fig. 6. Osbeckia aspera (L.) Blume: a. Habit; b. Inflorescence; c. Hypanthium; d. Bract; e. Calyx lobe; f. Intersepalar emergence; g. Stamens; h. Style; i. Seeds. 
other emergences restricted to the upper part of the hypanthium or absent; hairs short, bristly, subappressed, patent, curved upwards. Sepals triangular, 2-5 $\times 2-3.5 \mathrm{~mm}$, apex attenuate, 1 -nerved, hairy at apex, caducous. Petals obovate, 1-2.5 × 1-1.5 cm, pink to purple. Stamens 10; filaments $5-10 \mathrm{~mm}$ long; anthers narrowly ovate to oblong, 5-9 mm long, yellow, twisted, pore oblique on ventral side of the tip; connective with collar having one tubercle dorsally and another irregularly lobed one ventrally. Ovary united with hypanthium about half of its length; anther pockets extending to base of ovary; crown with short bristles; style 8-19 mm long, glabrous, curved; stigma slightly pointed. Capsules campanulate, 4-8 × 3-6 mm; seeds minute, 0.1-0.2 mm long, numerous, curved.

Flowering \& fruiting: October-March.

Chromosome number: $n=10$ (Meenakumari \& Kuriachan, 1990).

Habitat: Found on the margins of evergreen forests at an elevation above $500 \mathrm{~m}$ in association with $O$. virgata and O. parvifolia. Variations in size and appearance are noticed among populations at different altitudes. Plants growing in lower altitudes are generally tall with larger leaves whereas, those at higher altitudes are usually short with smaller leaves.

Distribution: India and Sri Lanka.

Specimens examined: INDIA, Kerala, Idukki district, Munnar, 13.05.1935, E. Barnes 118853 (DD); Palakkad district, Nelliyampathy, Karapara forest, Pothumalai, 19.12.1980, N.C. Nair \& P. Bharghavan 6928 (MH); Thiruvananthapuram district, Agasthyamala, Peppara, 08.03.1994, N. Mohanan \& T. Shaju 01973 (FRLH); Athirumala, Agasthyamala, 16.03.2016, Prashob \& Sibichen 6030 (DEV); Bonacaud, 16.03.2016, Prashob \& Sibichen 6029 (DEV); Ponmudi hills, 14.09.1977, N.C. Nair 51071 (MH); Ibid., 16.08.1980, M. Mohanan 69224(MH); Ibid., 13.12.2014, Prashob E Sibichen 5566 (DEV); Thrissur district, Karimala hills, 23.10.1990, N. Sasidharan 5736 (KFRI); Travancore, 28.08.1902, S.F. Baurdillon 1237/7104 (DD);
Vazhachal, 04.11.1989, N. Sasidharan 5500 (KFRI). Tamil Nadu, Coimbatore district, Attakatti, 06.07.1961, J. Joseph 12753 (MH); Dindigul district, Kodaikanal, Palamalai path, 10.05.1985, K.M. Matthew \& N. Rajendren 44923 (RHT); Kodaikanal, Puthur-Kavalapatty path, 02.05.1988, K.M. Matthew 52853 (RHT); Perumal peak, 14.12.1989, K.M. Matthew 53859 (RHT); Vandaravu-Marion shola Levinge path, 25.10.1988, K.M. Matthew 53634 (RHT); Kanyakumari district, Alagiapandiapuram, Mahendragiri hills, Kurusadi mottai, 24.09.1998, V.S. Manickam 17508 (XCH); Pechiparai, Kalasekaram range, 20.07.1975, S.P. Subramani 14620 (FRLH); Road to Karimoni, 26.02.1998, V.S. Manickam 14798 (XCH); Madurai district, Maharajamethu, 15.08.1990, V.Lakshmanan, 91166 (MH); Tirunelveli district, Inchikuzhi, 22.09.1988, R. Gopalan 88717 (MH); Kakachi, 12.10.1957, K.M. Sebastine 4416 (MH); Kakachi, Kothayar, 02.02.1997, V.S. Manickam 12250 (XCH); Kakachi to Sengaltheri path, 21.12.1996, V.S. Manickam 11840 (XCH); Kallar hills, 03.02.1998, V.S. Manickam 14707 (XCH); Ibid., 10.11.1959, K.M. Sebastine 9614 (MH); Kannikatti, 08.06.1957, K.M. Sebastine 409 (MH); Kudamadi hills, Mundanthurai, 26.06.1997, V.S. Manickam 13058 (XCH); Kuthiravetti shola, View point, 20.12.1996, V.S. Manickam 11796 (XCH); Manjanam parai, 30.05.1963, A.N. Henry 16377 (MH); Manjolai hills, 25.06.1957, K.M. Sebastien 3595 (MH); Martanamparai, 04.09.1962, A.N. Henry 17450 (MH); Muthukuzhi Vayal, 09.09.1976, A.N. Henry 48311 (MH); Ibid., 26.08.1976, A.N. Henry 47592 (MH); Sengaltheri, 09.03.1963, J. Joseph 15872 (MH); Upper Kothayar hills, 07.08.1996, V.S. Manickam 10049 (XCH); Vullar forest, Agastiar hills, 06.08.1998, V.S. Manickam 16838 (XCH).

Notes: Hansen (1977), treated O. travancorica and O. wightiana as varieties of $O$. aspera. However, the latter two are distinct in their habitat, altitudinal preferences, and micro-morphological characters and hence reinstated as distinct species in the present work. A comparison of morphological characters is provided in Table 2 . 
Table 2. Comparison of O. aspera, O. travancorica and O. wightiana

\begin{tabular}{|l|l|l|l|}
\hline Characters & O. aspera & O. travancorica & O. wightiana \\
\hline Leaves & Acrodromous, 3-nerved & $\begin{array}{l}\text { Acrodromous, 3-5-nerved } \\
\text { 5-7-nerved }\end{array}$ & Non-acrodromous, \\
\hline Hypanthium & Emergences absent & Emergences all over & Emergences all over \\
\hline Bracts & Ovate to cordate, 1-5 mm long & Cordate, 3-8 mm long & Ovate, 6-10 mm long \\
\hline Sepals & Triangular & Subulate & Subulate \\
\hline $\begin{array}{l}\text { Intersepalar } \\
\text { emergences }\end{array}$ & Sessile, ending in a tuft of hairs & $\begin{array}{l}\text { Stalked; stalk terete, hairy, } \\
\text { head stellate with a tuft } \\
\text { of bristles }\end{array}$ & $\begin{array}{l}\text { Stalked; stalk hairy, ending in } \\
\text { a small tuft of patent stellate } \\
\text { hairs }\end{array}$ \\
\hline Other emergences & Absent & Stalked, with a stellate head & Stalked, with a stellate head \\
\hline
\end{tabular}

Osbeckia gracilis Bedd., Madras J. Lit. Sci. ser. 3, 1: 45. 1864; Bedd. Trans. Linn. Soc. 24: 216. 1865; C.B.Clarke in Hook.f., Fl. Brit. India 2: 518. 1879; Gamble, Fl. Pres. Madras 1: 494. 1919; C.Hansen, Ginkgoana 4: 77. 1977; Mohanan, Fl. Quilon Dist. 187. 1984; M.Mohanan \& Henry, Fl. Thiruvanthapuram 198. 1994; Sasidh., Fl. Shenduruny W.L.S. 130. 1997; Sasidh., Fl. Periyar Tiger Reserve 144. 1998; Sasidh., Fl. Parambikulam W.L.S. 127. 2002. Type: INDIA, Kerala, Sispara Ghats, 4000 ft., s.d., Beddome 2975 (holo BM000944355 digital image!).

O. sublaevia Cogn. in A.DC. \& C.DC., Monogr. Phan. 7: 321. 1891; Gamble, Fl. Pres. Madras 1: 492. 1919. Type: INDIA, Tamil Nadu, Nilgiris, s.d., Perrottet 377 (holo BR0000005208722 digital image!).

O. lineolata Gamble., Kew Bull. Misc. Inform. 7: 241. 1918; Fl. Pres. Madras 1: 492. 1919. Lectotype (designated here): INDIA, South India, s.d., Sauliere 312 (K000867991 digital image!).

O. lineolata Gamble var. anamalayana G.S.Giri \& M.P.Nayar, Bull. Bot. Surv. India 25: 244. 1983. Type: INDIA, Tamil Nadu, Coimbatore (as Coimbatur), Kanalar, Anamalai Hills, 1950 m, 17.11.1980, M. Chandrabose 57793 (holo CAL!).

O. walkeri auct. Amitha Bachan \& Pradeep, Int. J. Adv. Res. 5(7): 339. 2017. non Arn., 1837. Fig. 7

Perennial, erect, branched shrubs, c. $1 \mathrm{~m}$ tall. Stems quadrangular, winged at angles, sparsely covered with short rigid hairs, hairs at nodes larger. Lamina lanceolate or oblanceolate, $2-7 \times 1-2.5 \mathrm{~cm}$, apex attenuate to acute, base attenuate, rarely acute, 3-5-nerved, both sides sparingly clothed with ascending short hairs, turns yellowish green when drying. Cymes terminal or sometimes axillary, 3-7-flowered. Flowers pentamerous; pedicels 0.5-2 mm long; bracts green, ovate, 5-10 mm long, ciliate, both sides spatially clothed with short hairs. Hypanthium campanulate, 3.5-5.5 × 3-5 mm, clothed with patent or ascending hairs with bullate base; intersepalar emergences rounded, tuberculoid with elongated slightly flattened tips ending in a tuft of bristles, caducous; other emergences usually absent. Sepals 5, triangular to oblong, 2-5.5 mm long, ciliate, with a very prominent midrib having appressed to patent hairs, apex with a tuft of long hairs, caducous. Petals 5, broadly obovate, 20-25 $\times 12-17 \mathrm{~mm}$, purple, ciliate. Stamens 10; filaments 7-12 mm long; anthers narrowly ovate to oblong, yellow, 7-9 mm long, twisted, pore slightly oblique on ventral side; connective with two small dorsal and two ventral tubercles. Ovary partially fused with hypanthium, usually exceeding hypanthium, anther pockets present, crown distinct in fruit; style $1.5-3 \mathrm{~cm}$ long. Capsules campanulate, 5-8 × 4.5-7 mm; seeds minute, $0.1-0.2 \mathrm{~mm}$ long, numerous, curved.

Flowering \& fruiting: September-December.

Chromosome number: Not reported. 


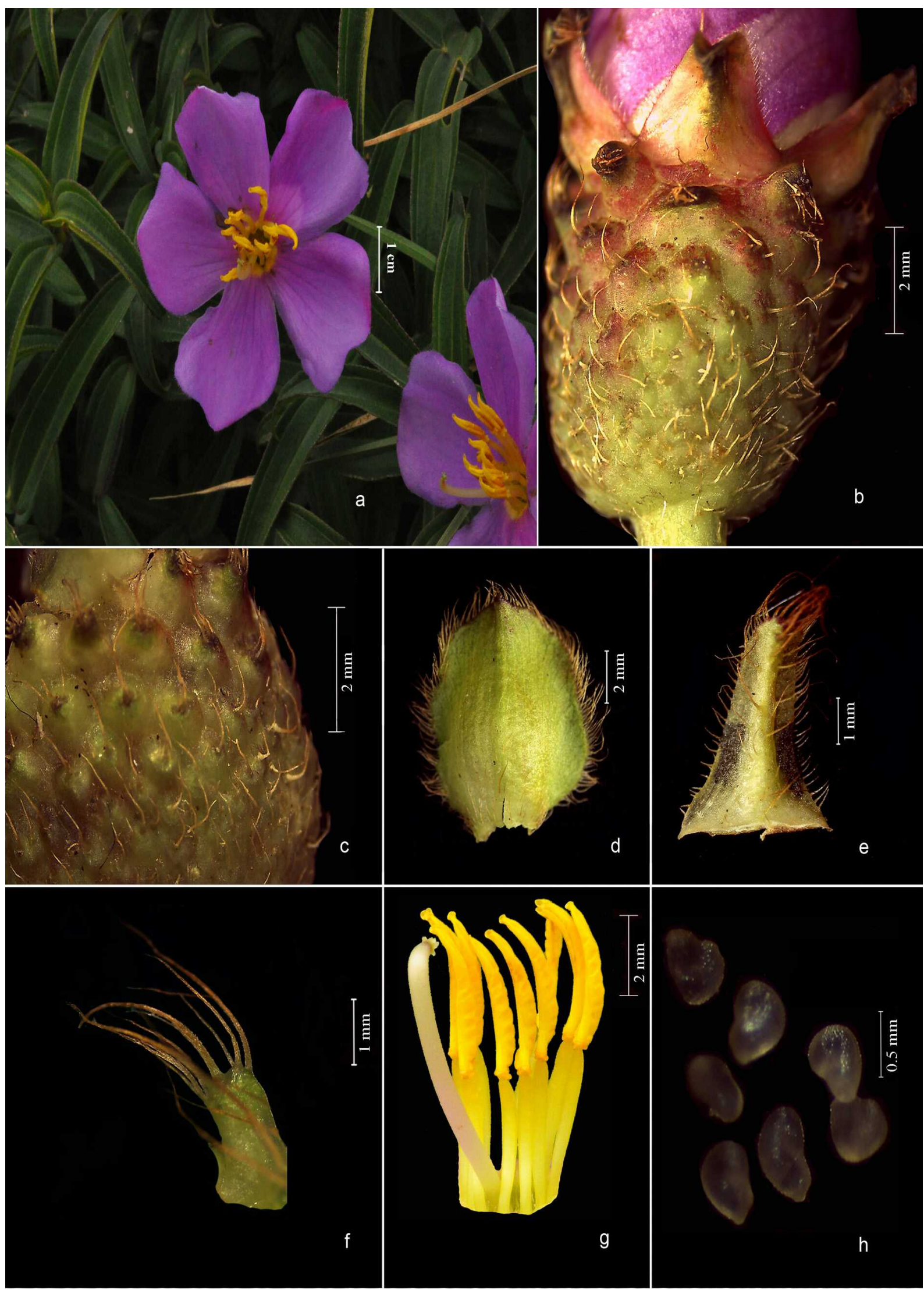

Fig. 7. Osbeckia gracilis Bedd.: a. Habit; b \& c. Hypanthium; d. Bract; e. Calyx lobe; f. Intersepalar emergence; g. Stamens; h. Seeds. 
Habitat: This species occurs commonly in borders of shola forests and in open grasslands, between 1000-2000 m elevation. Plants at lower altitude are characterized by their tall size, long inter nodes and pale green leaves whereas, those at higher altitude are shorter, having short internodes and dark green leaves. The species is usually seen associated with O. leschenaultiana and O. brachystemon.

Distribution: Endemic to India.

Specimens examined: INDIA, Kerala, Idukki district, Devikulam, 11.09.1968, D.B. Deb 62192 (MH); Munnar, Lockhurt gap, 10.10.1963, K.V. Sebastian 17480 (MH); Pamba range, 05.10.1976, K. Vivekananthan 48604 (MH); Upper Vagaruai, 16.11.1965, B.V. Shetty 62192 (MH); Palakkad district, Silent Valley N.P., Sispara, 13.10.2014, Prashob \& Sibichen 5543, 5544, 5545 (DEV); Silent Valley N.P., Wallakad station, 13.10.2014, Prashob E Sibichen 5547 (DEV). Tamil Nadu, Coimbatore district, Anamali, 12.09.1961, J.Joseph 13327 (MH); Berijam road to Periar path, 07.07.1986, K.M. Matthew \& N. Rajendren 45727 (RHT); Blackburne cliff, 10.08.1987, K.M. Matthew \& K.T. Mathew 50036 (RHT); Valparai, 06.09.1983, K. Ramamurty E R. Chandrasekaran 78431 (MH); Ibid., 23.03.1990, V.B. Hosagovsan 92472 (MH); Dindigul district, Kodaikanal-Leving, 09.08.1984, K.M. Matthew 40809 (RHT); Ibid., 03.09.1985, S.J. Britto 42147 (RHT); Kodaikanal-Leving path, 09.07.1986, K.M. Matthew \& N. Rajendran 45769 (RHT); Kodaikanal- Shembaganur path, 03.08.1985, K.M. Matthew 41634 (RHT); Korappur, 09.09.1986, K.M. Matthew \& M. Charles 46804 (RHT); Palani hills, Perumal peak, 12.12.1986, K.M. Matthew 47911 (RHT); Madurai district, Thenkikalparai, 25.08.1990, V. Lakshmanan 94321 (MH).

Notes: Analysis of populations from various localities has shown diversity in the indumentum pattern of O. gracilis. Hairs on the hypanthium are long and profuse in higher altitude specimens (from Sispara, Silent valley) whereas, it is short and sparse in lower altitude specimens (Munnar hills). Similarly, leaves are broadly ovate to lanceolate in lower altitude specimens whereas it is lanceolate among high altitude specimens. Irrespective of these variations, all populations are characterized by the brown, patent, curved, bristly hairs at least on the lower part of the hypanthium.

In the protologue of O. gracilis, Beddome (1864) described the flower as tetramerous with 8 anthers. However, pentamerous flowers with 10 stamens are noticed throughout the collections (including specimens from type locality).

Osbeckia leschenaultiana DC., Prodr. 3: 42. 1828. C.B.Clarke in Hook.f., Fl. Brit. India 2: 520. 1879; Gamble, Fl. Pres. Madras 1: 493. 1919; C.Hansen, Ginkgoana 4: 87. 1977; Swarup. et al., Shola For. Kerala 59. 1998; Sivar. \& Mathew, Fl. Nilambur 272. 1997; Sasidh., Fl. Periyar Tiger Reserve 145. 1998; Sasidh., Fl. Chinnar W.L.S. 131. 1999; Sasidh., Fl. Parambikulam W.L.S. 129. 2002; Mohanan \& Sivad., Fl. Agasthyamala 279. 2002. Type: INDIA, Tamil Nadu, Nilgiris (as Nellygerry), 1823, Leschenault 10, (holo G00310433 digital image!).

O. gardneriana Wight, Icon. Pl. Ind. Orient. 3: t.997. 1845. Lectotype (designated here): INDIA, Tamil Nadu, Nilgiris, Sispara, 04, Wight 1085 (M0165759 digital image!).

O. wightiana Benth. var. erythrotrica Benth. ex Wall., Numer. List 4060. 1831, nom. nud. Fig. 8

Perennial, erect shrubs, c. $1 \mathrm{~m}$ tall. Stems quadrangular, branched, covered with thin, patent, ascending, curved, brown to reddish hairs. Lamina ovate, $1.3-4.5 \times 0.8-2.5 \mathrm{~cm}$, sessile, dark green on upper side and light green on lower side, 3-5nerved, apex acute, base obtuse to subcordate, both sides thinly clothed with hairs. Inflorescence terminal, dense panicles of 5-7-flowers, subtended by 2-4 pairs of leaves, upper pair of leaves reduced to semi bracts; bracts orbicular, 3-6.5 $\mathrm{mm}$ broad, green, apex acute, margins ciliate, appressed-hairy dorsally. Flowers pentamerous; pedicels $2-5 \mathrm{~mm}$ long. Hypanthium campanulate, 8-13 × 5-9 mm, densely clothed with patent emergences and hairs; intersepalar emergences 1-3 mm long stalked; stalk terete with patent short hairs; head disc-like with stellate long hairs with thick bases, persistent in young fruit; other emergences similar but smaller; 


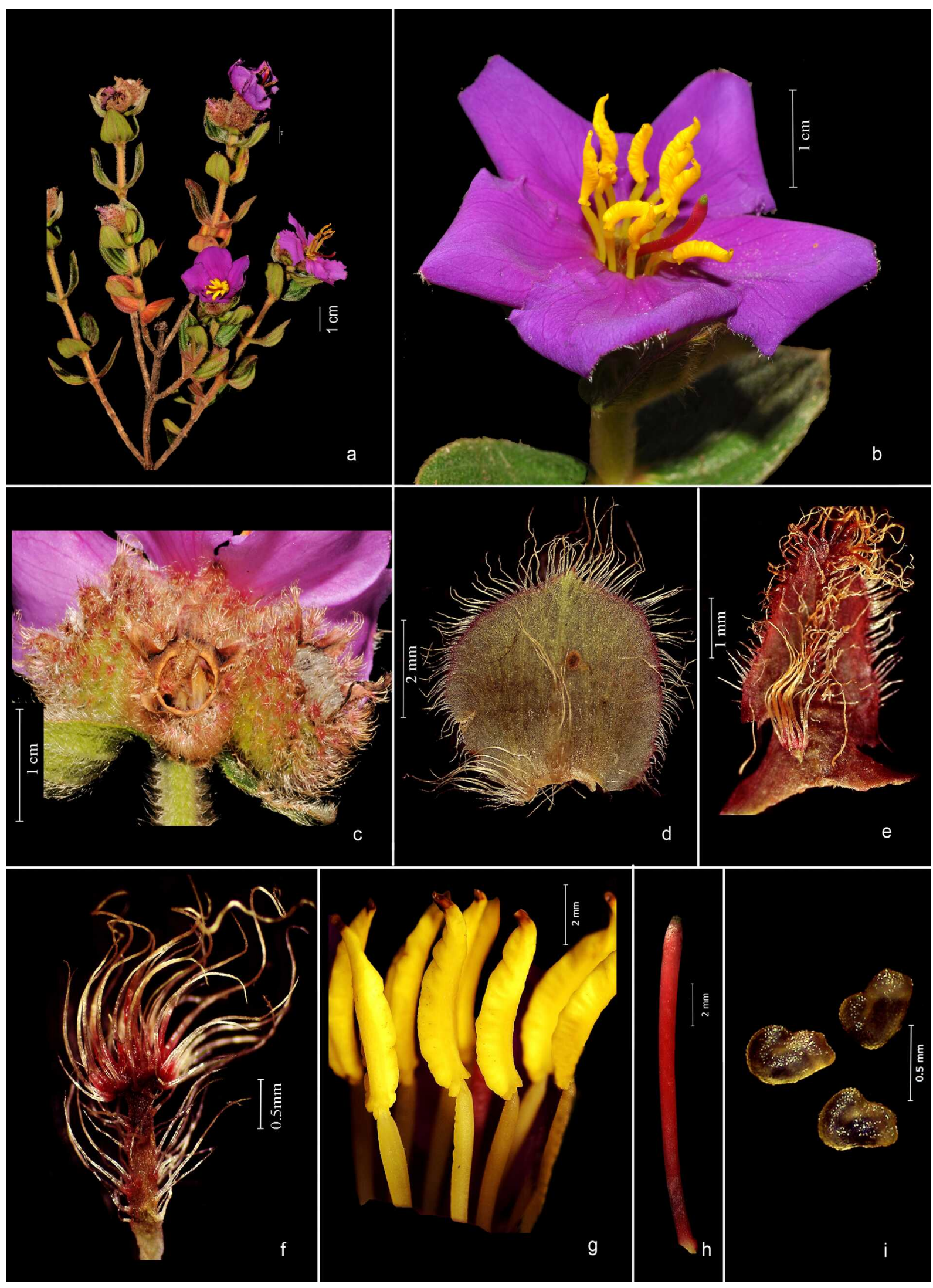

Fig. 8. Osbeckia leschenaultiana DC.: a. Habit; b. Flower; c. Hypanthium; d. Bract; e. Calyx lobe; f. Intersepalar emergence; g. Stamens; h. Style; i. Seeds. 
a few hairs present in between the emergences. Sepals triangular, 4-6.5 ×0.5-2 mm, narrowed into an oblong lobe, with acute to obtuse apex, margins ciliate, 1-nerved, with many patent hairs on nerves. Petals obovate, $15-22 \times 10-15 \mathrm{~mm}$, purple, apex ciliate. Stamens 10; filaments 5-8 mm long; anthers yellow, narrowly ovate to oblong, 5-8 mm long, twisted, pore oblique on ventral side; connective tuberculate with a small dorsal and a large ventral lobe. Ovary united with hypanthium for about half of its length, densely covered with hairs; anther pockets extending to base of ovary; crown hairy; style 12-18 mm long, glabrous. Capsules campanulate, $8-12 \times 5-9 \mathrm{~mm}$; seeds numerous, minute, $0.1-0.2 \mathrm{~mm}$ long, curved.

Flowering \& fruiting: August-December.

Chromosome number: Not reported.

Habitat: In higher elevations from 1800 to $2500 \mathrm{~m}$ above sea level, seen in forest borders and in open grasslands in association with $O$. reticulata, $O$. brachystemon and O. gracilis.

Distribution: Endemic to South India.

Specimens examined: INDIA, Kerala, Idukki district, Anamudi shola N.P., 10.11.2014, Prashob E Sibichen 5556, 5557, 5586 (DEV); Devikulam, 02.02.1970, B.V. Shetty 31788 (CAL, MH); Edamalakudi, 16.01.2015, Prashob E Sibichen 5587, 5589, 5591 (DEV); Eravikulam N.P., 16.01.2015, Prashob \& Sibichen 5593 (DEV); Eravikulam N.P., Pettimudi, 16.01.2015, Prashob \& Sibichen 5592 (DEV); Eravikulam N.P., Rajamala, 16.01.2015; Kulamavu, 12.10.1982, C.N. Mohanan \& V.S. Ramachandran 74592 (MH); Eravikulam Western slope, 04.12.1987, P. Bhargavan 87329 (CAL, MH); Mathikettan shola, 06.12.1986, K.M. Matthew $\&$ M. Charles 47752 (RHT); Kollam district, Shenduruny high range, 05.1937, E. Barnes 118851 (DD); Travancore hills, 09.1937, E. Barnes 112219 (DD). Tamil Nadu, Coimbatore district, Anamalai, Akkamalai Grass hills, 11.03.1978, D.K. Hore 657 (CAL); Dindigul district, Kodaikanal, 25.08.1898, A.G. Bourne (CAL); Ibid., 21.03.1956, Pallithanam 1576 (RHT); Ibid., 19.03.1950, Danial Sundaraj \&
Sankaran Rao 94117 (MH); Kodaikanal, Berijam slope above the shola, 24.07.1984, K.M. Matthew 40496 (RHT); Kodaikanal to Berijam road, 19.11.1985, K.M. Matthew 42860 (RHT); Kodaikanal, Berijam-Vembadi, 19.11.1985, K.M. Matthew 42739 (RHT); Kodaikanal, Kukkal, 08.04.1987, K.M. Matthew \& M. Charles 49087 (RHT); Kodaikanal, upper Palani, 16.08.1911, C.E.C.Fischer 2856 (CAL); Kodaikanal, Vandaravu, 09.01.1987, K.M. Matthew 48151 (RHT); Kodaikanal, Vandaravu main shola, 29.08.1987, K.M. Matthew 50553 (RHT); Pulney hills, 1869, Beddome 21180 (MH); Vandaravu peak, Kerala peak (Kerala border), 12.05.1985, K.M. Matthew 41430 (RHT); Nilgiri district, Bhavani, 19.12.1970, B.V. Shetty 37489 (MH); Keti, 24.01.1957, K.M. Sebastine 2206 (CAL); Kotagiri-Ooty road, 26.04.1971, N.C. Rathakrishnan 38086 (MH); Kottabettu, 25.02.1970, A.A. Ansari 1046 (CAL); Mullimand-Avalanche, 26.12.1970, B.V. Shetty 37605 (MH); Nilgiri, 20.03.1870, C.B. Clarke 111188 (CAL); Ooty, 10.1910, A. Meebold 11867 (CAL); Perumal hills, 12.04.1987, C. Sudharsan 281 (CAL); Pykara river bank, 12.09.1930, V. Narayanaswamy 4245 (MH); Sispara, 05.1889, J.S. Gamble 21174 (MH); Tirunelveli district, way to Pothagiri, 05.02.1989, R. Gopalan 88727 (MH).

Notes: Plants found in higher altitudes are short (c. $0.5 \mathrm{~m})$, profusely branched with very short internodes (c. $5 \mathrm{~mm}$ long) and those found in lower altitudes are tall $($ c. $1 \mathrm{~m})$, sparsely branched with long internodes (c. 3-5 cm long).

Osbeckia mehrana G.S.Giri \& M.P.Nayar, Kew Bull. 41(2): 429. 1986. Type: INDIA, Kerala, Thiruvananthapuram, way to Chemungi, $1200 \mathrm{~m}$, 08.03.1979, M. Mohnan 61706 (holo CAL0000015811!).

Fig. 9

Perennial, erect, woody shrubs, c. $3 \mathrm{~m}$ tall. Stems branched, quadrangular, densely clothed with long, brown ascending or sub-patent hairs. Petioles 7-13 mm long, clothed with thin ascending hairs; lamina ovate, $1.5-4.5 \times 0.8-3 \mathrm{~cm}, 5$-nerved, dark green, apex acute, base obtuse, margins entire, upper side with ascending to sub patent hairs, lower side 


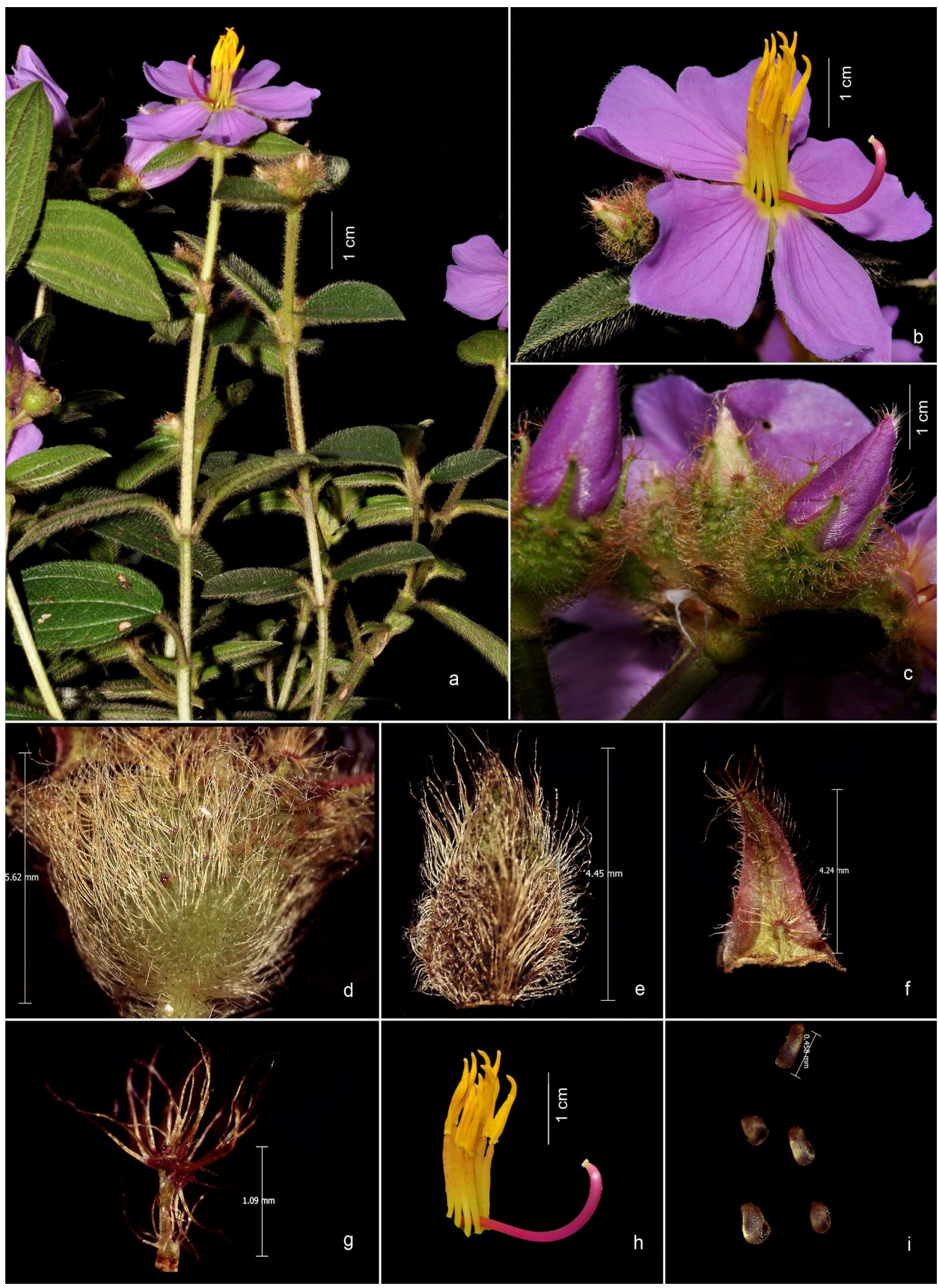

Fig.9. Osbeckia mehrana G.S. Giri \& M.P. Nayar: a. Habit; b. Flower; c \& d. Hypanthium; e. Bract; f. Calyx lobe; g. Intersepalar emergence; h. Stamens \& Style; i. Seeds. 
with patent hairs, hairs on the nerves are longer. Inflorescence terminal and axillary panicles, 5-7flowered; bracts ovate, 2.5-4.5 × 1-2.5 mm, green, margins ciliate, wooly dorsally, caducous; Flowers pentamerous, pedicels $2-5 \mathrm{~mm}$ long. Hypanthium campanulate, 4-6 × 3-5 mm, densely clothed with patent hairs and stellate emergences, sometimes emergences absent; intersepalar emergences stalked 1-2 mm long, stalk terete with ascending hairs, head disc-like with stellate long hairs, caducous; other emergences similar but smaller. Sepals 5, triangular, 3-5 × 1.5-2 mm, with acute apex, 1nerved, margins ciliate, hairs at apex longer, midrib prominent with long patent hairs dorsally. Petals 5, obovate, 11-23 × 6-8 mm, pink, margins ciliate. Stamens 10; filaments 4-6 mm long, glabrous; anthers narrowly ovate, $4.5-5.5 \mathrm{~mm}$ long, yellow, pore oblique on ventral side of the tip; connective extended to a small collar with small dorsal and ventral lobe. Ovary 3-4.5 mm long, united with hypanthium for about half of its length; anther pockets extending to base of ovary, with dense cover of ascending hairs; crown with ascending hairs; style 12-17 mm long, glabrous, curved; stigma capitate, papillose. Capsules campanulate, 6-10 × 6-8 mm, enclosed by the calyx tube; seeds minute, numerous, $0.1-0.2 \mathrm{~mm}$ long, curved and muricate.

Flowering \& fruiting: November-April.

Chromosome number: $n=10$ (Meenakumari \& Kuriachan, 1990).

Habitat: Occurs in evergreen forests and in grasslands at an elevation above $1500 \mathrm{~m}$., in association with $O$. aspera var. wightiana and $O$. tirunelvelica.

Distribution: Endemic to South India.

Specimens examined: INDIA, Kerala, Thiruvananthapuram district, Agasthyamala, Chemungi, 08.03.1979, M. Mohanan 61706 (CAL); Agasthyamala, Pongalapara, 15.03.2016, Prashob \& Sibichen 6025, 6026, 6028 (DEV); near Natarikal, Travancore boundary, 04.03.1917, s.coll. $21111(\mathrm{MH})$; Poonkulam, on the way to
Agastyarkudam, 05.03.1980, M. Mohanan 115996 (MH). Tamil Nadu, Tirunelveli district, Kothayar Dam house, 16.04.1997, V.S. Manickam 12532 (XCH); Kothayar Hills, Dam valley, 12.03.1998, V.S. Manickam 14994 (XCH); Kothayar, Muthukuzhivayal, 11.03.1998, V.S. Manickam 14944 (XCH); Kothayar Hills, Stream across Dam house, 10.03.1998, V.S. Manickam 14878 (XCH); Papanasam hills, Uppuma mottai, 07.04.1999, V.S. Manickam 18856 (XCH).

Notes: This taxon is morphologically allied to $O$. gracilis but can be easily distinguished by dense, long hairs on stem and leaves, hypanthium with emergences and stellate headed intersepalar emergences. But in O. gracilis the stems and leaves are thinly covered with hairs and the hypanthium is without emergences.

Osbeckia nepalensis Hook., Exot. Fl. 1(2): t. 31. 1822; C.B.Clarke in Hook.f., Fl. Brit. India 2: 521. 1879; C.Hansen, Ginkgoana 4: 67. 1977; N.P.Balakr, Fl. Jowai \& Vicinity 205. 141. 1983. Lectotype (designated by Hansen, 1977): NEPAL, Katmandu, J.E. Smith s.n. (K000867999 digital image!).

O. chulesis D.Don, Prodr. Fl. Nepal. 221. 1825. Type: NEPAL, Narainhetty, 27.07.1802, Hamilton s.n. (holo BM 000521788 digital image!).

Melastoma nepalensis Lodd., Bot. Cab. 8: t. 707. 1823, nom. nud.

Melastoma chulesis Ham. ex D.Don, Prodr. Fl. Nepal. 221. 1825, pro. syn.

O. speciosa D. Don, Prodr. Fl. Nepal. 222. 1825, nom. illeg.

Perennial, erect, branched shrubs, c. $2 \mathrm{~m}$ tall. Stems clothed with appressed to ascending or shaggy hairs. Leaves sessile or sub sessile (petioles 0.5-1.5 $\mathrm{mm}$ long); lamina lanceolate, 5-16 × 2-5.5 cm, 57-nerved, apex acute, base cordate or subcordate, both sides clothed with ascending shaggy hairs above, soft beneath. Inflorescence terminal or axillary panicles, 5-10-flowered. Flowers pentamerous; pedicels1- $6 \mathrm{~mm}$ long; bracts ovate 
to broadly ovate, $7-15 \times 5-9 \mathrm{~mm}$, ciliate and appressed-hairy. Hypanthium campanulate, 6-10 $\times 5.5-7 \mathrm{~mm}$, with few to several subappressed scale like emergences, simple hairs absent; intersepalar emergences 1-1.5 × 1-2 mm, lobed, with a tuft of short bristles, caducous; other emergences similar in shape. Sepals 5 , ovate, $8-11 \times 4-7 \mathrm{~mm}$, apex often acute, with few indistinct nerves, margins ciliate, caducous. Petals 5 , broadly obovate to obcordate, 15-20 × 4-7 mm long, pink, margins ciliate. Stamens 10; filaments 4-6 mm long; anthers narrowly ovate, 6-9 $\mathrm{mm}$ long, attenuate, not twisted, pore oblique on ventral side at the apex, connective sometimes slightly prolonged between collar and sacs. Ovary united with hypanthium for about half of its length; anther pockets extending to the base of ovary; crown distinct; style 13-19 mm long. Capsules campanulate, 6-10 × 5-8 mm, rim of hypanthium exceeding the crown; seeds minute, curved, 0.1-0.2 mm long, numerous.

\section{Key to the varieties}

1a. Petals pink or rarely purple...... var. nepalensis

1b. Petals white var. albiflora

Osbeckia nepalensis Hook. var. nepalensis, Exot. Fl. 1(2): t. 31. 1822.

Fig. 10

Flowering \& fruiting: June-December.

Chromosome number: $n=19$ (Favarger, 1962), $n=$ 20 (Mehra \& Khosla, 1969).

Habitat: Common in open grasslands of higher altitudes (500-1500 m) and in swampy areas of evergreen forests.

Distribution: Bhutan, China, India (Arunachal Pradesh, Assam, Meghalaya, Manipur, Nagaland, West Bengal and Sikkim), Laos, Malaya, Myanmar, Nepal, North Vietnam and Thailand.

Specimens examined: INDIA, Arunachal Pradesh East Siang district, Pasighat, Pangin, 12.07.2015, Prashob \& Hareesh 6121 (DEV); Lohit district, 28.09.1969, A.S. Rao 48174 (CAL); Subansiri district, Kameng F.D., Kalaktang, 14.01.1958, G. Panigrahi 15515(CAL); Nyapin, 21.11.1964, A.R.K.
Sastry 40837 (ASSAM); Pagba, 11.17.1974, C.L. Malhotra 55927 (ASSAM); Tirap district, Bimalpur, 07.09.1958, G. Panigrahi 17044 (ASSAM); West Kameng district, Kalaktang, 14.11.1973, P.K. Hajra 54112 (ASSAM); Sessa Orchid Sanctuary, Tippi, 01.07.2015, Prashob E Sibichen 6118 (DEV). Assam, Darrang district, Bornadi W.L.S., 19.03.2012, N. Sarma 124700 (ASSAM); Goalpara district, Goalpara, 08.09.1937, G.K. Deka 17057 (ASSAM); Kachugaon, 02.1947, M.M. Srinivasan 21937 (ASSAM); Ultapani, 27.12.1975, B.N.M. E party 5583 (ASSAM); Kamrup district, Digboi gorhat, 11.07.1959, G. Panigrahi 19002 (ASSAM, CAL); Ghagrachuk, 18.06.1964, A.S. Rao 38964 (ASSAM); Ghagrachuk forest, 18.07.1964, A.S. Rao 38946 (CAL); Kimini river, 23.11.1957, G. Panigrahi 11476 (ASSAM, CAL); Kokrajhar district, Chakrasila W.L.S., 04.11.2006, Ranjit Daimary 111929 (ASSAM); Newgong district, Belsiri river, 22.07.1958, G. Panigrahi 14219 (CAL); Dhoka R.F., 25.08.1964, N.P. Balakrishnan 39424 (ASSAM, CAL); Kohora, 22.09.1957, R.S. Rao 9779 (CAL); Upper Dikking river, 08.07.1957, G. Panigrahi 18746 (ASSAM, CAL). Manipur, Kangpokpi district, Laimakhong, 31.08.1952, D.B. Deb 594 (CAL); Lakhama, 03.1906, A. Meebold 5604 (CAL). Meghalaya, East Khasia hills district, Barapani road, 11.09.1970, A.S. Rao 38634 (CAL); Barapani, 13.10.1954, G.K. Deka 11425 (ASSAM); Cherrapunji, 21.12.1956, G. Panigrahi 4959 (CAL); East Khasi hills, Pommora, 07.09.2011, Santhosh Nampy 4725 (DEV). Karhan, 05.10.1948, S.K. Mukherjee 3617 (CAL); Ibid., 08.1880, C.E.C. Fischer 172171 (CAL); Khasia, 12.1887, H. Collett 172173, 172171 (CAL); Khasia-Bhilasa, 06.08.1886, C.B. Clarke 172175 (CAL); Khasia \& Jaintia Hills, 25.08.1956, G. Panigrahi 3003 (CAL); Ibid; 25.08.1918, H.H. Haines 172210 (CAL); Ibid., 25.08.1956, R.S. Rao 3003 (ASSAM, CAL); Ibid; 21.12.1956, G. Panigrahi 4959 (ASSAM); Ibid; 09.10.1930, S. Sharma 2565 (ASSAM); Ibid., 23.01.1931, S. Sharma 9002 (ASSAM); Lockhart hills, 1905, Abu Hassan 167 (CAL); Makum, 21.11.1911, I.H. Burkill 35744 (CAL); Pynursla, 12.09.2015, Prashob \& Sibichen 6131, 6132 (DEV); 


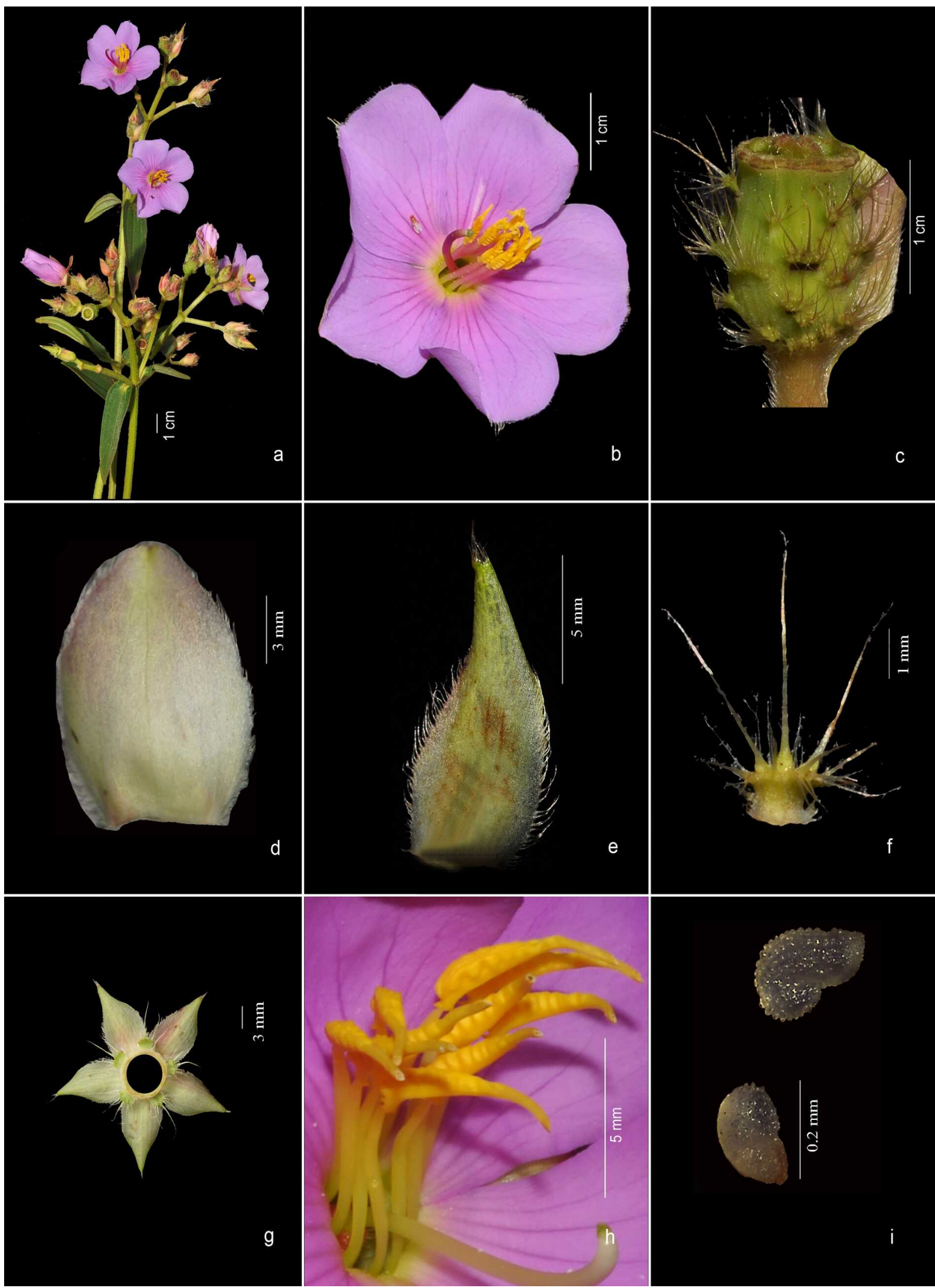

Fig. 10. Osbeckia nepalensis Hook. var. nepalensis: a. Habit; b. Flower; c. Hypanthium; d. Bract; e. Calyx lobe; f. Intersepalar emergence; g. Calyx lobes \& intersepalar emergences; $\boldsymbol{h}$. Stamens; i. Seeds. 
Tipperah, Champamoora hills, 23.10.1915, P.M. Debbarman 172212 (CAL); Jaintia hills district, Jowai, 18.11.1969, N.P Balakrishnan 49990 (ASSAM); Jarain pitcher plant garden, 19.09.2015, Prashob \& Sibichen 6144 (DEV); Jowai to Jarain, 16.08.1968, N.P. Balakrishnan 42961 (ASSAM); Nongtalang village, 19.09.2015, Prashob \& Sibichen 6147 (DEV); Garo hills district, Garo hills, 01.1903, J. Marten 172193 (CAL); Napak area, 19.09.1975, M.K.V. Rao 64181 (ASSAM); Raling, 21.08.1968, N.P. Balakrishnan 46892 (ASSAM); Ibid., s.d.,T.D. Srinivasan 246 (CAL); Songsak R.F., 29.11.2006, R. Shanprne 113114 (ASSAM); Ri-Bhoi district, Nongpoh, 07.1949, B.B. Sagma 22779 (ASSAM). Nagaland, Kohima district, Dzulake valley, Naga hills, 09.1935, N.L. Bor 6161(DD); Naga hills, 09.1886, Prain 172189 (CAL); Ibid., 09.1939, N.L. Bor 90571 (DD); Ibid., 07.1944, N.L. Bor 21001 (ASSAM); Ibid., 11.1979, R.R. Fernandes 008 (DD);. Sikkim, East Sikkim district, Baluwa khani khola, 04.08.1980, P.K Hajra 271 (CAL, BSHC); Chumbi valley, 12.1904, G.L. Searight 0096 (CAL); Dikchu, 08.09.1997, B.K. Singh 19603 (BSHC); East Sikkim, 1862, T. Anderson 172198 (CAL); Ibid., 06.10.1875, C.B. Clarke 6073 (CAL); Ibid, 11.1900, Prain's collector 58 (CAL); ; Ibid., 08.1903, Prain's collector 169 (CAL); Faramsa, 08.10.1986, S. Singh 4500 (BSHC); Gangtok, 06.08.1981, B. Krishna 1659 (BSHC); Gangtok, Lower Sinchey, 09.09.1981, B. Krishna 1696 (BSHC); Kalpana Nag, 01.17.1876, G. King 187 (CAL); Rung Beliver Valley, 22.09.1908, W.W. Smith 61 (CAL); Ruspo, 14.11.1998, S.S. Dash 20961 (BSHC); Saramsa, 23.08.1980, P.K. Hajra 598 (BSHC); Tarku, 21.09.1968, N.C. Majumdar \& R.N. Banerji 583 (CAL); Taring to Dikchu, 07.09.1989, N.R. Mandal 11336 (BSHC). North Sikkim district, Dzangu, 24.11.1995, Sujit Kumar Janna 17955 (BSHC); Lingza, 14.09.1986, B. Mitra 6868 (BSHC); Nampik, 12.09.2000, D. Maity 23124 (BSHC); Rangrang bridge, Mangan-Rangrang Bridge, 25.08.2002, S.K. Rai 20586 (BSHC); Ibid., 17.09.2016, Prashob, Sibichen \& Anilkumar 6616 (DEV); Ibid.,09.09.1968, N.C. Majumdar \& R.N. Baner 341 (CAL); Singhik, 21.10.1984, B. Krishna
3237 (BSHC); Tingbong, 14.08.1989, N.R. Mandal 10163 (BSHC); South Sikkim district, Kewzing, 18.08.1985, A.K. Verma 4231 (BSHC); Sakyong to Pentong, 16.09.1986, A.K. Verma 7070 (BSHC); Sulphuk, 13.12.1985, A.K. Verma 4948 (BSHC); West Sikkim district, Dentam, 29.08.1997, P. Singh 16338 (BSHC); Dantam to Pelling, 13.09.2016, Prashob, Sibichen \& Anilkumar 6604 (DEV). West Bengal, Darjeeling district, Darjeeling, 06.1880, J.S. Gamble 749 (DD); Ibid., 09.1880, J.S. Gamble 172187 (CAL); below Mangpoo, 20.02.1914, s.coll. 40646 (CAL); between Sukia \& Siliguri, 07.1957, N.L.H. Krauss 323 (CAL); Jalpaiguri district, Kathambari, New Jalpaiguri, 25.04.1962, S.K. Mukarji 5550 (CAL); Kalimpong district, Kalimpong, 11.1879, J.S. Gamble 4000 (DD); Missiva compound, 10. 1913, H.J.C. Kinghoran 172177 (CAL); Nemati, 18.11.1999, G.S. Giri 20486 (CAL); New Jalpaiguri, 26.08.1912, N.C. Banargi 4048 (DD); Rajavathkhawa forest, 05.09.1981, B. Safui \& Party 10359 (CAL); s.loc. 08.12.1995, S. Chandra \& S.K Mandal 1080 (CAL).

Notes: Osbeckia nepalensis can be recognized easily by its large sessile leaves with cordate or subcordate base and the large scale-like intersepalar emergences on the hypanthium with a tuft of bristles. It closely resembles O. wynaadensis, but can be distinguished by the characteristic cordate or subcordate leaf base, as opposed to obtuse leaf base in the latter. Moreover, both are allopatric in distribution.

Osbeckia nepalensis Hook. var. albiflora Lindl., Edwards's Bot. Reg. 17: t.1475. 1831. Lectotype (designated here): Edward's Bot. Reg. 17: t.1475.

Fig. 11

Perennial, erect, branched shrubs, c. $2 \mathrm{~m}$ tall. Stems clothed with appressed to ascending or shaggy hairs. Leaves sessile or sub sessile (petioles 0.5-1.5 mm long); lamina lanceolate, 5-16 × 2-5.5 cm, 57-nerved, apex acute, base cordate or subcordate, both sides clothed with ascending shaggy hairs above, soft beneath. Inflorescence terminal or axillary panicles, 5-10-flowered. Flowers pentamerous; bracts ovate to broadly ovate, 7-15 $\times 5-9 \mathrm{~mm}$, ciliate and appressed-hairy; pedicels 1- 


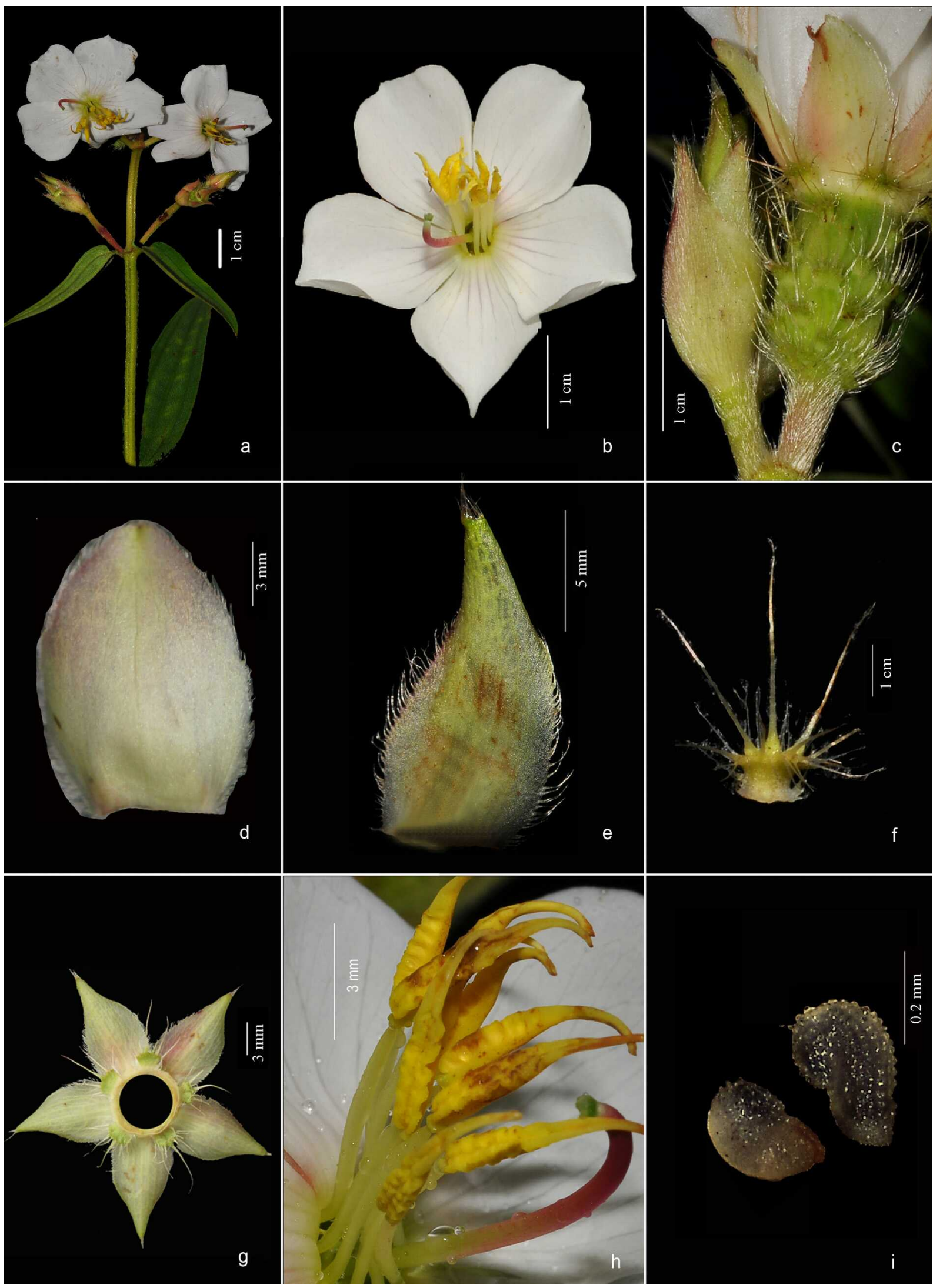

Fig. 11. Osbeckia nepalensis Hook. var. albiflora Lindl.: a. Habit; b. Flower; c. Hypanthium; d. Bract; e. Calyx lobe; f. Intersepalar emergence; g. Calyx lobes \& intersepalar emergences; h. Stamens; i. Seeds. 
$6 \mathrm{~mm}$ long. Hypanthium campanulate, 6-10 × 5.5$7 \mathrm{~mm}$, with few to several sub appressed scale like emergences, simple hairs absent; intersepalar emergences 1-1.5 × 1-2 mm, lobed, with a tuft of short bristles, caducous; other emergences similar in shape. Sepals 5 , ovate, $8-11 \times 4-7 \mathrm{~mm}$, apex often acute, with few indistinct nerves, margins ciliate, caducous. Petals 5, broadly obovate to obcordate, 15-20 × 4-7 mm long, white, margins ciliate. Stamens 10; filaments 4-6 mm long; anthers narrowly ovate, 6-9 $\mathrm{mm}$ long, attenuate, not twisted, pore oblique on ventral side at the apex, connective sometimes slightly prolonged between collar and sacs. Ovary united with hypanthium for about half of its length; anther pockets extending to the base of ovary; crown distinct; style 13-19 $\mathrm{mm}$ long. Capsules campanulate, $6-10 \times 5-8 \mathrm{~mm}$, rim of hypanthium exceeding the crown; seeds minute, curved, 0.1-0.2 mm long, numerous.

Flowering \& fruiting: June-December.

Chromosome number: $n=19$ (Favarger, 1962), $n=$ 20 (Mehra \& Khosla, 1969).

Habitat: Occurs in swampy areas of evergreen forests while in high altitudes it is seen in open grasslands.

Distribution: Bhutan, China, India, Laos, Malaya, Myanmar, Nepal, North Vietnam and Thailand.

Specimens examined: INDIA, Arunachal Pradesh, East Siang district, Kameng F.D., Kalaktang, 14.01.1958, G. Panigrahi 15615 (CAL); West Kameng district, Kalaktang, 14.11.1973, P.K. Hajra 54102 (ASSAM); Sessa Orchid Sanctuary, Tippi, 01.07.2015, Prashob \& Sibichen 6110 (DEV). Meghalaya, East Khasi hills district, Barapani, 13.10.1954, G.K. Deka 1435 (ASSAM); Khasia \& Jaintia Hills, 21.12.1956, G. Panigrahi 4949 (ASSAM); Pynursula, 12.09.2015, Prashob E Sibichen 6133 (DEV); Shillong, 23.01.1931, Shriram Sharma 9003(ASSAM); Jaintia hills district, Jowai, 18.11.1969, N.P. Balakrishnan 49989 (ASSAM). Sikkim, West Sikkim district, Dantam to Pelling, 13.09.2016, Prashob, Sibichen \& Anilkumar 6105 (DEV); Khecheopalri lake, 14.09.2016, Prashob, Sibichen \& Anilkumar 6609 (DEV).
Osbeckia nutans Wall. ex C.B.Clarke in Hook.f., Fl. Brit. India 2: 521. 1879; C.Hansen, Ginkgoana 4: 75. 1977; N.P.Balakr., Fl. Jowai 206. 141. 1983; Hajra \& Verma, Fl. Sikkim 296, 1996; Noltie, Fl. Bhutan 1994. O. nepalensis Hook. var. nutans (Wall. ex C.B.Clarke) D. Maity, Econ. Taxon. Bot. (27) 1235. 2003. syn. nov. Lectotype (designated by Hansen, 1977): INDIA, s.loc., s.d., Wallich 4068 (K000867997 digital image!).

O. nutans Wall., Numer. List 144, no. 4068. 1831, nom. nud.

Fig. 12

Perennial, erect, branched shrubs, c. $1.5 \mathrm{~m}$ tall. Stems covered with appressed hairs. Petioles 1-3 mm long; lamina narrowly lanceolate, 3-6.5 × 0.5$1 \mathrm{~cm}, 3$-nerved, apex acute, base obtuse, glabrous throughout except on nerves below. Panicles terminal, 4-6-flowered, subtended by a pair of reduced leaves. Flowers pentamerous; pedicels 3-5 $\mathrm{mm}$ long; bracts green, ovate to cordate 4-7 $\mathrm{mm}$ long, both sides spatially clothed with short hairs. Hypanthium campanulate, 4-4.5 × 4-4.5 mm, glabrous or with appressed scale like emergences having hairs at apex; intersepalar emergences exactly resembles normal emergences, both are caducous. Sepals 5, triangular, 6.5-7.5 mm long, with a few indistinct nerves, minutely ciliate, otherwise glabrous, caducous. Petals 5, obovate, 13-16 mm long, pink or purple, apex ciliate. Stamens 10; filaments 4-6 mm long; anthers narrowly ovate to oblong, 3-6 mm long, not twisted, pore oblique on ventral side of the tip; connective slightly prolonged into an undulating lobed collar. Ovary united with hypanthium; anther pockets extending to base of ovary, free part densely covered with appressed hairs; crown small, distinct, with short hairs; style 13-14 mm long. Capsules campanulate, 6.5-8.5 × 5-6.5 mm; seeds minute, curved, 0.1$0.2 \mathrm{~mm}$ long, numerous.

Flowering \& fruiting: August-March.

Chromosome number: Not reported

Habitat: Occurs in swampy areas and river margins.

Distribution: Bhutan, India Myanmar and Nepal. 


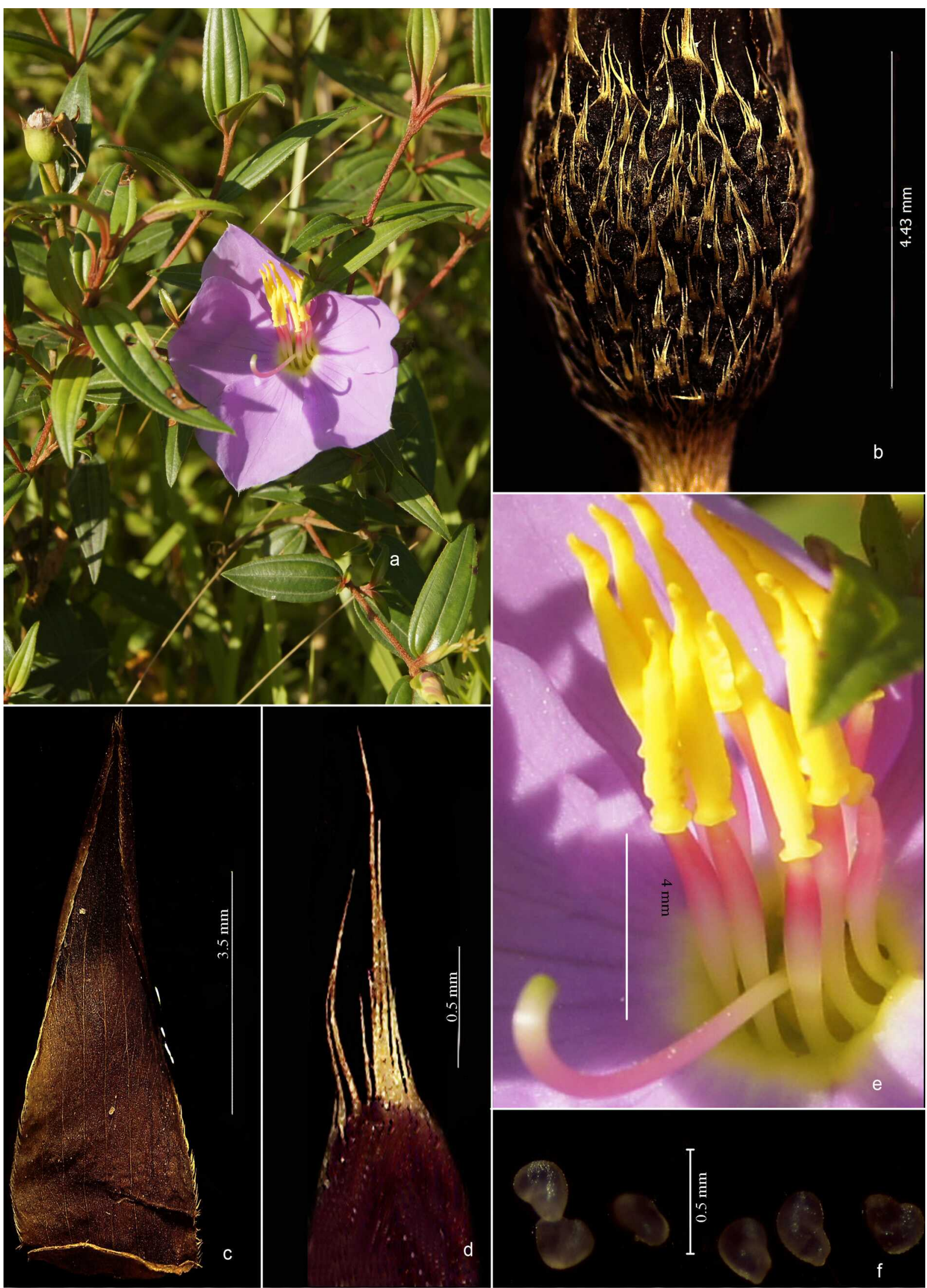

Fig. 12. Osbeckia nutans Wall. ex C.B. Clarke: a. Habit; b. Hypanthium; c. Calyx lobe; d. Intersepalar emergence; e. Stamens; f. Seeds. 
Specimen examined: INDIA, Arunachal Pradesh, Changlang district, Namdapha National Park, 10.06.1982, J. Joseph 81114 (ASSAM); Vijaynagar, 26.12.1996, Das Chauhan 99555 (ASSAM); Lohit district, Chamba village, 17.12.1969, A.S. Rao 48687 (CAL, ASSAM); Deopani river, north of Roing, 03.10.1969, A.S. Rao 48210 (CAL, ASSAM); Zunglua-Rusa, 05.09.1958, G. Panigrahi 16775 (CAL); Papum Pare district, Kimin to Zero, 23.11.1957, G. Panigrahi 11465 (ASSAM); Subansiri district, Subansiri Reserve forest Kimin-Kumin, 24.09.1959, G. Panigrahi 19302 (CAL, ASSAM); West Kameng district, Chaku, 21.04.1957, G. Panigrahi 6307 (CAL, ASSAM); Champari, 03.12.1916 H. Haines s.n. (CAL); Kameng, s.d., A.S. Rao 50761 (ASSAM); Kameng, Chopai forest camp, 18.02.1974, A.S. Rao 56443 (ASSAM); Orchid Research centre, Tippi, 30.06.2015, Prashob \& Sibichen 6117 (DEV); Shukhanala, s.d., P.K. Hajra 54483 (ASSAM); Sissini to foot hill camp, 18.04.1957, G. Panigrahi 7042 (ASSAM); Tippi to Balupara 16.09.2016, Prashob \& Arun 6117 (DEV); Tirap district, Zunghua-Rusa, 05.09.1958, G. Panigrahi 16775 (ASSAM). Assam, Goalpara district, s.loc., 03.12.1918, Upendranath Kanjilal 7450 (ASSAM); Kokrajhar district, Chakoarila W.L.S, 03.07.2003, Ranjit Daimary 118075 (ASSAM); Lakhimpur district, Kimin river, 23.11.1957, G. Panigrahi 11465 (CAL, ASSAM); North Lakhimpur, Naharani Reserve, 17.05.1966, D.M. Verma 46390 (ASSAM); Subansiri Reserve forest Lakhimpur, 24.09.1959, G. Panigrahi 16775 (CAL); Upper Assam, Dikhow river forest, 30.03.1895, s.coll. 11093 (CAL). Manipur, Chamoo, 14.01.1882, George Watt 172153 (CAL); Naga hills, 12.1907, A. Meebold 7339 (CAL). Meghalaya, East khasi hills district, Shillong, s.d. Griffith s.n. (CAL); Garo hills district, Napak lake, 19.07.1976, M.K.V. Rao 64614 (ASSAM); Jaintia hills district, 17.12.1911, I.H. Burkill s.n. (CAL); West Jaintia Hills, Nartiang, 28.08.1968, N.P. Balakrishnan 47194 (ASSAM). Sikkim, East Sikkim district, Bumbing on the way to Rangto, 19.12.1981, P. Chakrabarty 2001 (BSHC); s.loc. 18.12.1903, Prain 25 (CAL); East Sikkim, 25.07.1985, D.C.S. Raju
3921 (BSHC); $20^{\text {th }}$ Mile side, Bordang, 17.11.2001, S.S. Dash E S.K. Rai 23920 (BSHC); East Sikkim, 24.11.1980, S.B. Ram 00870 (CAL); Rangpo, Namthang, 19.12.1981, P. Chakrabarty 867 (CAL); Rhenock, 24.11.1980, S.B. Ram 00841 (BSHC); Rongly, 07.12.1980, P. Chakrabarty 1030 (CAL); Reshi, 02.01.1996, S.K. Jana 18013 (BSHC); way to Rongli, 07.12.1980, P. Chakrabarty 1030 (BSHC); South Sikkim district, Teesta valley, 15.11.1901, Prain s.n. (CAL); West Sikkim district, Labdang, 11.1990, Dr. Prain 60 (CAL); Sikkim, 10.1874, C.B. Clarke 28650 (CAL).

Notes: Osbeckia nutans can be recognized by its lanceolate leaves and large sepals exceeding the hypanthium. It is interesting to note that both glabrous or hypanthium with scaly emergences are found on the same plant.

Maity (2003) treated O. nutans as a variety of O. nepalensis. But critical examination of the type, protologue and live specimens led us to reinstate its original species status. Leaves are small (3-6.5 × $0.5-1 \mathrm{~cm})$, narrow, petiolate and glabrous in $O$. nutans whereas it is large $(5-16 \times 2-5.5 \mathrm{~cm})$, sessile, with shaggy hairs in O. nepalensis.

Osbeckia reticulata Bedd., Trans. Linn. Soc. 25: 216. 1865; C.B.Clarke in Hook.f., Fl. Brit. India 2: 520. 1879; Gamble, Fl. Pres. Madras 1: 493. 1919; C.Hansen, Ginkgoana 4: 115. 1977. Lectotype (designated by Hansen, 1977): INDIA, Tamil Nadu, Coimbatore, Anamallais, s.d., Beddome 2998 (BM000944342 digital image!).

O. alveolata Bedd., Icon. Pl. Ind. Or. 1: 37. 168. 1874. Type: INDIA, Tamil Nadu, Coimbatore, Anamallais, 6-7000 ft, s.d., Beddome 239 (holo K000867948 digital image!).

O. reticulata Bedd., Madras J. Lit. Sci. ser. 3(1): 45. 1864, nom. nud.

Fig.13

Perennial, woody shrubs or small trees, 2-7.5 m tall; stems branched; young branches quadrangular with dense cover of patent hairs and brownish bristles. Petioles 7-18 mm long; lamina ovate, 4$10 \times 2.5-6 \mathrm{~cm}, 5-7-n e r v e d$, acrodromous, apex acute, base subcordate, margins reticulate, upper 


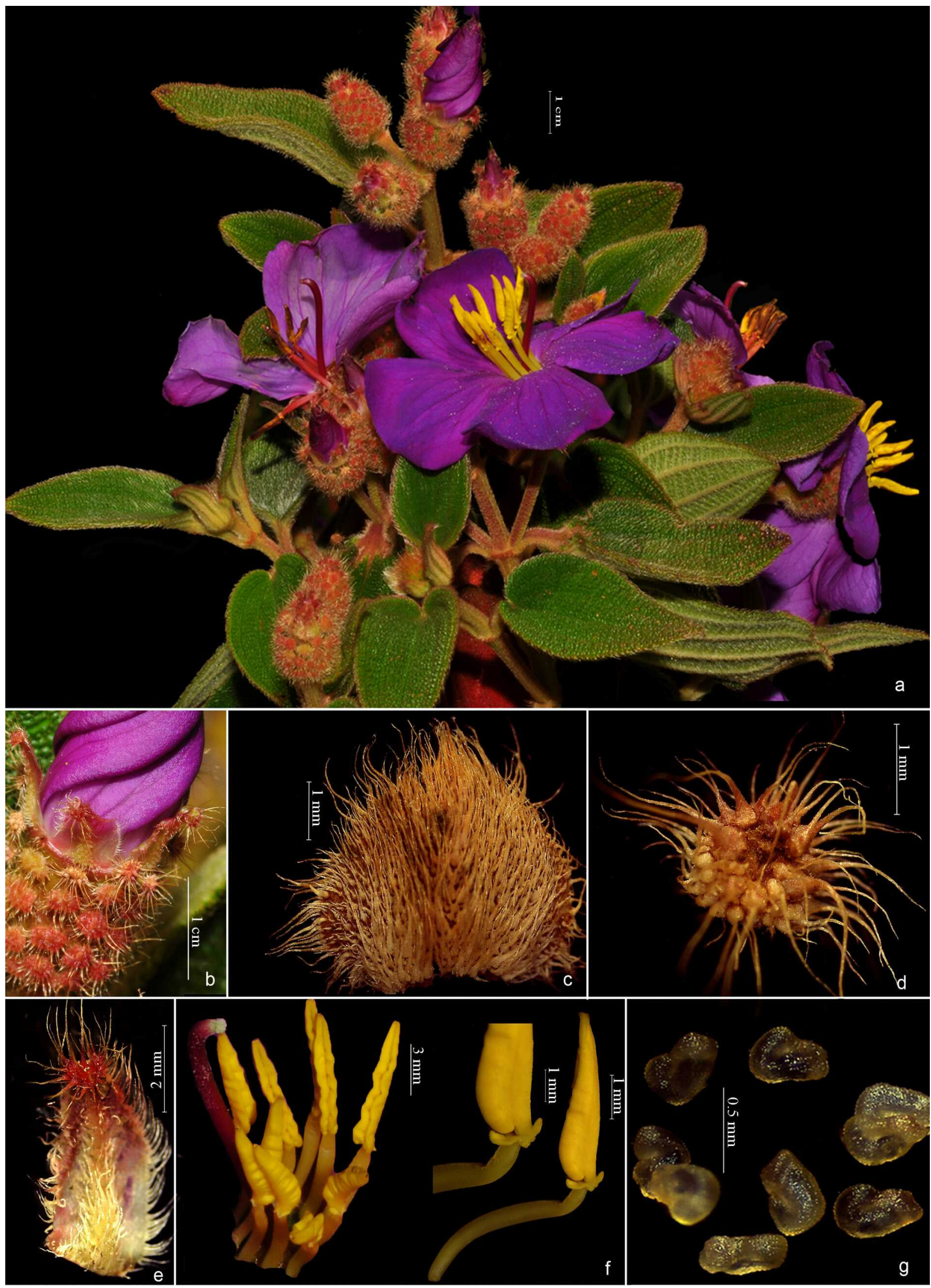

Fig. 13. Osbeckia reticulata Bedd.: a. Habit; b. Hypanthium; c. Bract; d. Intersepalar emergence; e. Calyx lobe; f. Stamens; g. Seeds. 
side bullate, densely covered with ascending, incurved and bristly hairs, lower side with patent hairs. Inflorescence a terminal cluster of cymes, 3-9-flowered. Flowers pentamerous; pedicels c. 5 $\mathrm{mm}$ long; bracts brownish, cordate, 4-6 $\times 5-7 \mathrm{~mm}$, margins ciliate, densely appressed hairy dorsal. Hypanthium campanulate, 9-13 × 8-9 mm, covered with large emergences with hairs; intersepalar emergences short, having many patent strong curved bristles, caducous; other emergences few, similar. Sepals 5, triangular, 4-7 × 1.5-3 mm, prominently 1 -nerved with patent hairs on nerves, margins ciliate, caducous. Petals 5, obovate, 2$3.5 \times 1.4-2.5 \mathrm{~cm}$, purple, apex ciliate. Stamens 10; filaments 7-12 mm long; anthers narrowly ovate, 8-11 mm long, yellow, not twisted, beak absent, pore single, more or less oblique on dorsal side, connective with conspicuous collar having two small dorsal lobes or tubercles and two large ventral lobes. Ovary united with hypanthium about half of its length; anther pockets extending to base of ovary; crown distinct with groups of united hairs; style 16-22 mm long, glabrous; stigma capitate. Capsules campanulate, 9-12 × 8-11 mm, ribbed; seeds numerous, curved.

Flowering \& fruiting: November-April.

Chromosome number: $n=10$ (Meenakumari \& Kuriachan, 1990).

Habitat: Occurs in shola forests and grass lands above $1000 \mathrm{~m}$.

Distribution: Endemic to South India.

Specimens examined: INDIA, Kerala, Idukki district, Anamudi shola N.P., Methap, 09.11.2014, Prashob \& Sibichen 5554, 5555 (DEV); Eravikulam N.P., 15.01.2015, Prashob \& Sibichen 5580, 5581, 5582, 5583, 5584, 5585 (DEV); Palakkad district, Perumalmudi, 21.04.1970, s.coll. 559 (MH). Tamil Nadu, Dindigul district, Bear shola, Kodaikanal, 07.03.1987, K.M. Matthew \& Rajendren 48545 (RHT); Kodaikanal, 19.03.1950, Daniel Sundaraj E Sakharan Rao 93805 (MH); Poomparai, Kodaikanal, 22.04.1965, Ramamurthy 23362 (MH); Poomparai, 09.04.1987, K.M. Matthew \& Charles
49116, 49146 (RHT); Ibid., 20.05.1987, K.M. Matthew \& Charles 49577 (RHT); Kodaikanal, 19.03.1956, Pallithanam 1551 (RHT); Kodaikanal, St. Mary's road, 10.05.1979, K.M. Matthew 16634 (RHT); Berijam, Kodaikanal, 19.11.1985, K.M. Matthew 42755 (RHT); Pambar shola, Kodaikanal, 07.02.1986, K.M. Matthew 44103 (RHT); Ibid., 29.04.1986, K.M. Matthew \& Rajendren 44696 (RHT); Vandaravu peak, Kodaikanal, 24.01.1998, K.M. Matthew 55124 (RHT).

Notes: This species is distinct from other taxa by its small tree-like habit, 7-9-nerved, large bullate leaves with minutely reticulate margin. The emergences on hypanthium are also unique.

Osbeckia tirunelvelica Manickam \& Murugan ex Kottaim. \& Gnanasek., Phytotaxa 231(3):300. 2015. Type: INDIA, Tamil Nadu, Tirunelveli District, Manjolai hills, Road below Manjolai, s.d., Manickam \& Murugan 15109 (holo XCH!).

O. tirunelvelica Manickam \& Murugan, J. Econ. Taxon. Bot. 25(3): 626. 2002, nom. inval. Fig. 14

Perennial, erect, branched shrubs, c. $1 \mathrm{~m}$ tall. Stems subterete, sparsely scabrous. Petioles 5-10 mm long; lamina eliptic to lanceolate, $2-6.5 \times 1.5-3 \mathrm{~cm}$, 3-5-nerved, green; apex acute to acuminate, base acute, both sides thinly clothed with patent hairs. Inflorescence terminal, condensed cyme or cluster of 6-9-flowers or 2-3 such cymes together, panicle subtended by bracts and a pair of leaves; bracts green, small ovate to broadly ovate, 1-3 mm long, ciliate and densely appressed hairy. Peduncle $2-5 \mathrm{~mm}$ long. Flowers pentamerous. Hypanthium campanulate, $4-8 \times 2-4 \mathrm{~mm}$, with a thin cover of simple hairs below and tufted hairs above; intersepalar emergences 1-2.5 mm long, oblong, stalked, with terminal and lateral hairs; other emergences restricted to the upper part of the hypanthium, or absent; hairs short, bristly, subappressed, patent, curved upwards. Sepals 5, subulate, 2-5 × 2-3.5 mm, prominently 1-nerved, margins ciliate with long hairs at apex, a few patent hairs on nerves, persistent in fruit. Petals 5, obovate, $1-2.5 \times 1-1.5 \mathrm{~cm}$, pink to purple. Stamens 10; 

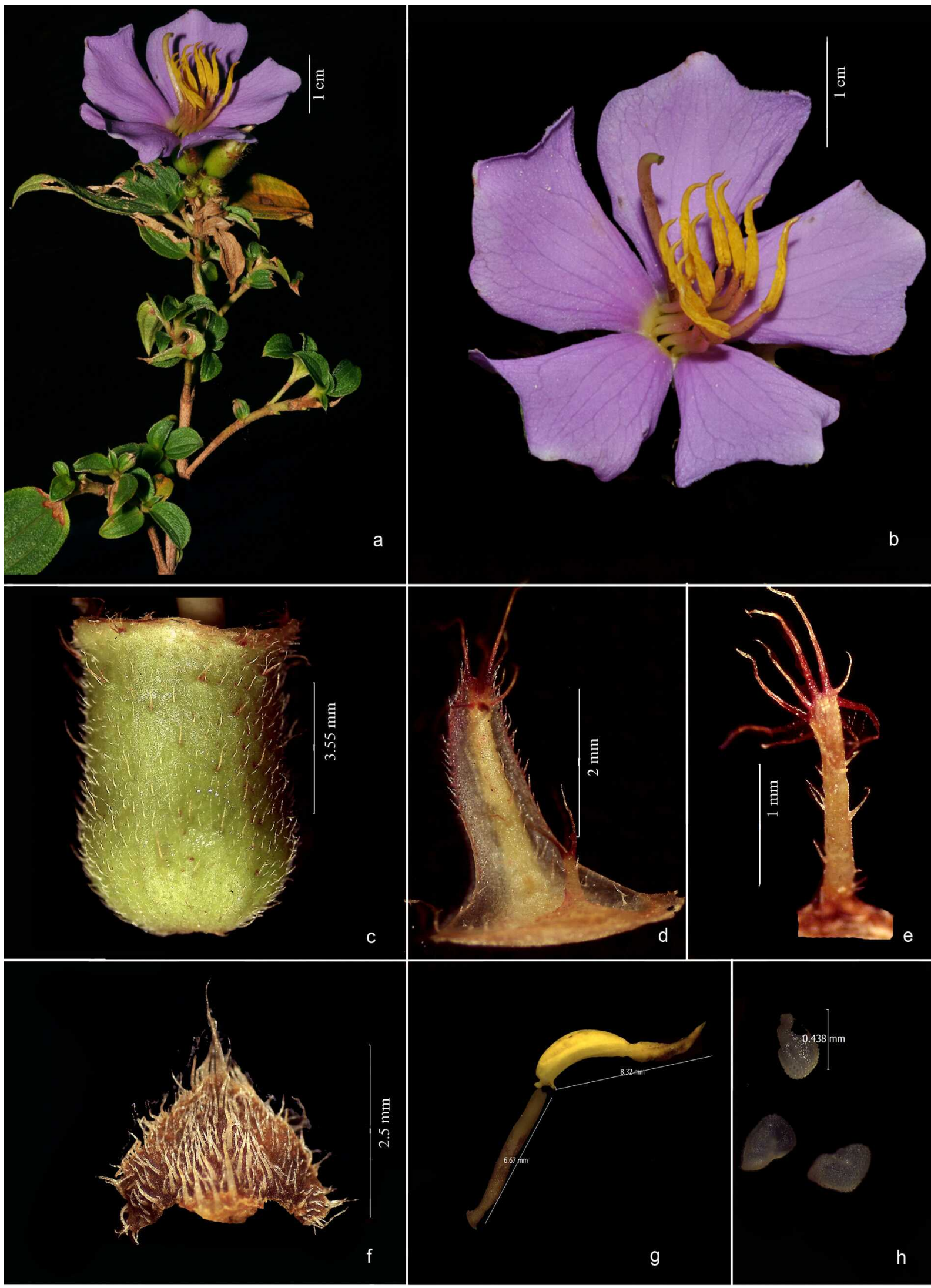

Fig. 14. Osbeckia tirunelvelica Murugan \& Manickam ex Kottaim. \& Gnanasek.: a. Habit; b. Flower; c. Hypanthium; d. Calyx lobe; e. Intersepalar emergence; f. Bract; g. Stamen; h. Seeds. 
filaments 5-10 $\mathrm{mm}$ long; anthers narrowly ovate to oblong, twisted, yellow, 5-9 mm long, pore oblique on ventral side of tip; connective with collar having one tubercle dorsally and slightly or irregularly lobed ventrally. Ovary united with hypanthium about half of its length, anther pockets extending to base of ovary; crown with short bristles, style 8-19 mm long, glabrous, curved; stigma slightly pointed. Capsules campanulate, 4-8 × 3-6 mm; seeds minute, 0.1-0.2 mm long, numerous, curved.

\section{Flowering \& fruiting: August-January.}

Chromosome number: Not reported

Habitat: In evergreen forests and tea plantations at an elevation above $750 \mathrm{~m}$ in association with $O$. aspera and O. mehrana.

\section{Distribution: Endemic to India.}

Specimens examined: INDIA, Tamil Nadu, Tirunelveli district, Manjolai hills $\pm 1115 \mathrm{~m}$, 06.01.2016, Prashob \& Sibichen 6023 (DEV); Tirunelveli, Kothayar \pm 1206 m, 06.01.2016, Prashob \& Sibichen 6025 (DEV); Tirunelveli, on the way from Kakachi to Ooth $\pm 1161 \mathrm{~m}$, 06.01.2016, Prashob \& Sibichen 6027 (DEV).

Notes: The publication of O. tirunelvelica by Manickam and Murugan (2002) is invalid as two collections were simultaneously indicated as "type" in the protologue. The name was later validated by Kottaimutu and Gnanasekaran (2015) following Art. 40 of ICN (Turland et al., 2018).

Osbeckia travancorica Bedd. ex Gamble, Bull. Misc. Inform. Kew 1919(10): 404. 1919; Gamble, Fl. Pres. Madras 493. 1919. O. aspera (L.) Blume var. travancorica (Bedd. ex Gamble) C.Hansen, Ginkgoana 4: 84. 1977. syn. nov.; Mohanan, Fl. Quilon Dist., 187. 1984; Babu, Fl. Malappuram Dist. 257. 1990; M.Mohanan \& Henry, Fl. Thiruvanthapuram 198. 1994; Sasidh. \& Sivar., Fl. Pl. Thrissur For. 190. 1996; Sivar. \& Mathew, Fl. Nilambur 272. 1997; Sasidh., Fl. Periyar Tiger Reserve 144. 1998; Sasidh., Fl. Parambikulam W.L.S. 127. 2002; Anil Kumar et al., Fl.
Pathanamthitta 222. 2005. Type: INDIA, Kerala, Travancore, 23.11.1851, Wight 1100 (holo K000867975 digital image!).

Fig. 15

Perennial, erect, shrubs, 1-2 m tall. Stems branched, quadrangular; sparsely hirsute, hairs dense at nodes. Petioles 2-6 mm long; lamina ovate to lanceolate, 4-11 × 1-4.5 cm, green, 3-5-nerved, apex acute to attenuate, base obtuse, both sides thinly clothed with patent hairs. Inflorescence terminal panicles to cluster of cymes, 5-8-flowered. Flowers pentamerous; pedicels 4-7 mm long; bracts green, cordate, 3-8 mm long, margins ciliate, densely appressed-hairy. Hypanthium campanulate, 5-10 $\times 3-6 \mathrm{~mm}$, densely covered with emergences, bristles and hairs; intersepalar emergences stalked, 1-3 mm long, green turns brown, stalk terete, base flat with patent hairs, head stellate with tuft of bristles, caducous; other emergences smaller. Sepals 5, subulate, 2-5 × 1-4 mm; symmetrical, prominently 1-nerved, patent hairs occur on the midrib dorsally, margins ciliate, apex with caducous stellate emergences. Petals 5, obovate, 13-20 × 10$17 \mathrm{~mm}$, pink to purple, margins ciliate. Stamens 10; filaments 6-10 mm long; anthers narrowly ovate to oblong, yellow, 5-11 mm long, twisted; connective prolonged into a collar with a dorsal and a slightly lobbed ventral tubercle. Ovary united with hypanthium for about half of its length; style 8-12 mm long. Capsules campanulate, 6-12 $\times$ 4-9 mm; seeds minute, numerous, $0.1-0.2 \mathrm{~mm}$ long, curved.

Flowering \& fruiting: September-March.

Chromosome number: $n=10$ (Meenakumari \& Kuriachan, 1990).

Habitat: Found in evergreen forest margins at an elevation above $300 \mathrm{~m}$, commonly seen in association with O. virgata and O. abrahamii.

Distribution: Endemic to South India.

Specimens examined: INDIA, Kerala, Ernakulam district, Neriamangalam, 01.12.1993, E.S. Santhosh kumar 18389 (FRLH); Idukki district, Bison valley, Munnar, 09.1937, E. Barnes 118824 (DD); Devikulam, 12.1919; A. Meebold 13191 (CAL); 


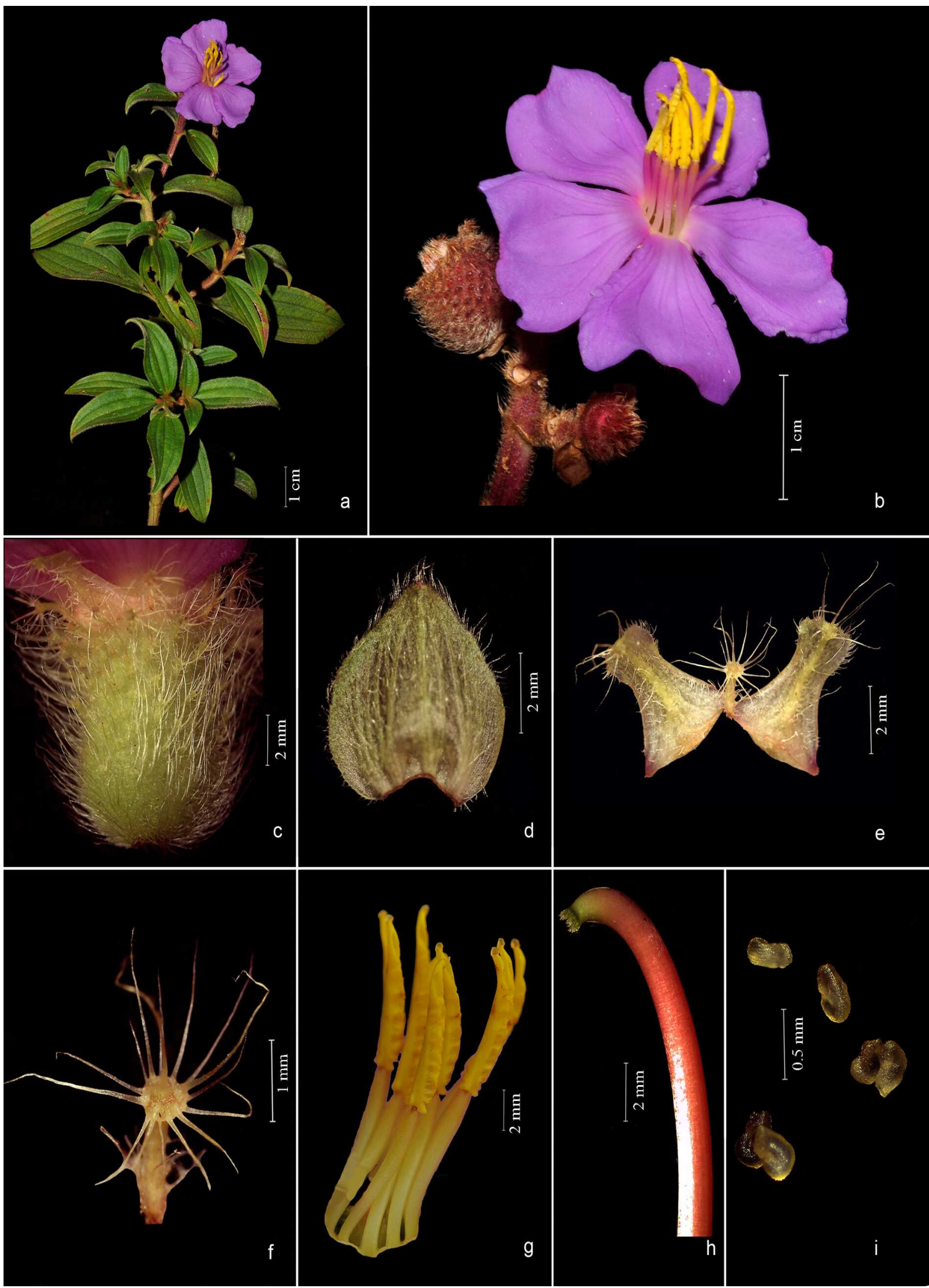

Fig. 15. Osbeckia travancorica Bedd. ex Gamble: a. Habit; b. Inflorescence; c. Hypanthium; d. Bract; e. Calyx lobes \& intersepalar emergence; f. Intersepalar emergence; g. Stamens; h. Style; i. Seeds. 
Devikulam, 24.03.1980, K. Ramamurthy 66344 (CAL); Kattappana, 08.11.1981, B. Ramanujan \& C.N. Mohanan 72415 (MH); Ibid., 27.09.1981, B. Ramanujan \& C.N. Mohanan 7203 (CAL); Kurisuhmala, 20.11.2014, Prashob \& Sibichen 5564 (DEV); Meemutty, 12.12.1982, C.N. Mohanan 76018 (MH); Microwave station, 08.11.1981, V.S. Raju E V.S. Ramachndran 71207 (CAL); Vagamon, 20.11.2014, Prashob E Sibichen 5562 (DEV); Vazhikkadavu, Pindimedu, 02.10.1996, P. Bhargavan 92055 (MH); Kollam district, Mukkapuzha, 22.12.1972, C.N. Mohanan 59605 (MH); Kottayam district, Kangazha, 29.09.1989, V.T. Antony 48476 (MH); Kurisumala, 08.11.1984, V.T. Antony 930 (CAL); Palakkad district, Mukkali forest office, 05.11.1976, E. Vajravelu $94523(\mathrm{MH})$; Muthikulam, Shiruvani, 02.03.1970, A A. Ansari 1070 (CAL); Nillical, Silent Valley, 23.11.1937, N.L. Bor 9161 (DD); Pathanamthitta district, Pamba to Anathode, 16.12.1974, K. Vivekananthan 45316 $(\mathrm{MH})$; Thiruvananthapuram district, Ponmudi, 17.11.1977, M. Mohanan 52541 (CAL); Poonkulam, Agastyarkudam, 05.03.1981, M. Mohanan 66049 (CAL); Thrissur district, Athirapally, 14.10.1994, N. Sasidharan 686 (FRLH); Thumboormuzhi, 12.09.1976, K. Ramamurthy 48476 (MH). Tamil Nadu, Coimbatore district, Anaimali hills, Pariuipare, 02.04.1912, C.E.C. Fischer 172106 (CAL); Dindigul district, Kodaikanal, 16.08.1911, C.E.C. Fischer 2865 (CAL); Pillar rock, 14.09.1968, D.B. Deb 30847 (CAL); Upper Palani, 16.08.1911, C.E.C. Fischer s.n. (CAL); Kanyakumari district, Upper Kothayar, s.coll. 53370 (CAL); Nilgiris district, Coonor, 06.1889, J.S. Gamble 21437; Ibid., 11.1889, J.S. Gamble, 13246; Ibid., 02.1899, Prains 13246 (CAL); Gudalur, Naduvattam, 18.01.1961, B.V. Shetty 1191 (CAL); Kotagiri Aravenu, 04.01.1957, K. Subramanyam 1888 (CAL); Upper Tiger shola, 19.01.1957, K. Subramanyam 2069 (CAL).

Notes: Hansen (1977) reduced O. travancorica Bedd. ex Gamble as a variety of O. aspera and remarked that the variety is distinct due to the asymmetrical calyx lobe and curved strong bristles on the stem.
After verifying the protologue and examining the type, and illustration on it by Gamble, we came to the conclusion that $O$. travancorica Bedd. ex Gamble is a distinct species, which can be very well distinguished by the downwardly curved, strong, bristles on the stem and uniform triangular sepals with obtuse or slightly emarginate apex.

Osbeckia virgata D.Don ex Wight \& Arn., Prodr. Fl. Ind. Orient. 1: 323. 1834; C.Hansen, Ginkgoana 4: 107. 1977; Mohanan, Fl. Quilon Dist. 188. 1984; Ansari, Fl. Kasaragod Div. 165. 1985; Manilal, Fl. Silent Valley 111. 1988; Antony, Syst. Stud. Fl. Kottayam Dist. 167. 1989; Babu, Fl. Malappuram Dist. 259. 1990; M.Mohanan \& Henry, Fl. Thiruvananthapuram 199. 1994; Sasidh. \& Sivar., Fl. Pl. Thrissur For. 190. 1996; Sasidh., Fl. Shenduruny W.L.S. 131. 1997; Sivar. \& P. Mathew, Fl. Nilambur 274. 1997; Sasidh., Fl. Periyar Tiger Reserve 145. 1998; Sasidh., Fl. Chinnar W.L.S. 132. 1999; Mohanan \& Sivad., Fl. Agasthyamala 280. 2002; Pull. et al., Fl. Eastern Ghats 59. 2002; Sasidh., Fl. Parambikulam W.L.S. 129. 2002; Anil Kumar et al., Fl. Pathanamthitta 223. 2005; Sunil \& Sivadasan, Fl. Alappuzha Dist. 297. 2009. Asterostoma virgata (D.Don ex Wight \& Arn.) Blume, Mus. Bot. 1(4): 50. 1849. Lectotype (designated by Hansen, 1977): INDIA, s.loc, s.d., Wight 1147B (E00174614 digital image!).

O. cupularis D. Don ex Wight \& Arn., Prodr. Fl. Indiae Or. 1: 323. 1834, pro parte; C.B.Clarke in Hook.f., Fl. Brit. India 2(6): 514. 1879; Gamble, Fl. Pres. Madras 1: 494. 1919. Type: INDIA, Kerala, Thiruvananthapuram, 03.1827, Wallich 4074 (holo K001038091 digital image!).

O. octandra auct. C.B.Clarke in Hook.f., Fl. Brit. India 2(6): 521. 1879. non DC., 1828; Gamble, Fl. Pres. Madras 1: 493. 1919; Manilal \& Sivar., Fl. Calicut 110. 1982; Subram., Fl. Thenmala Div. 141. 1995.

Melastoma glomeratum J.G.Klein ex Wight \& Arn., Prodr. Fl. Ind. Orient. 1: 323. 1834 , pro.syn.

O. wightiana Benth., Numer. List . No. 4074. 1831, nom. nud.

Fig.16 


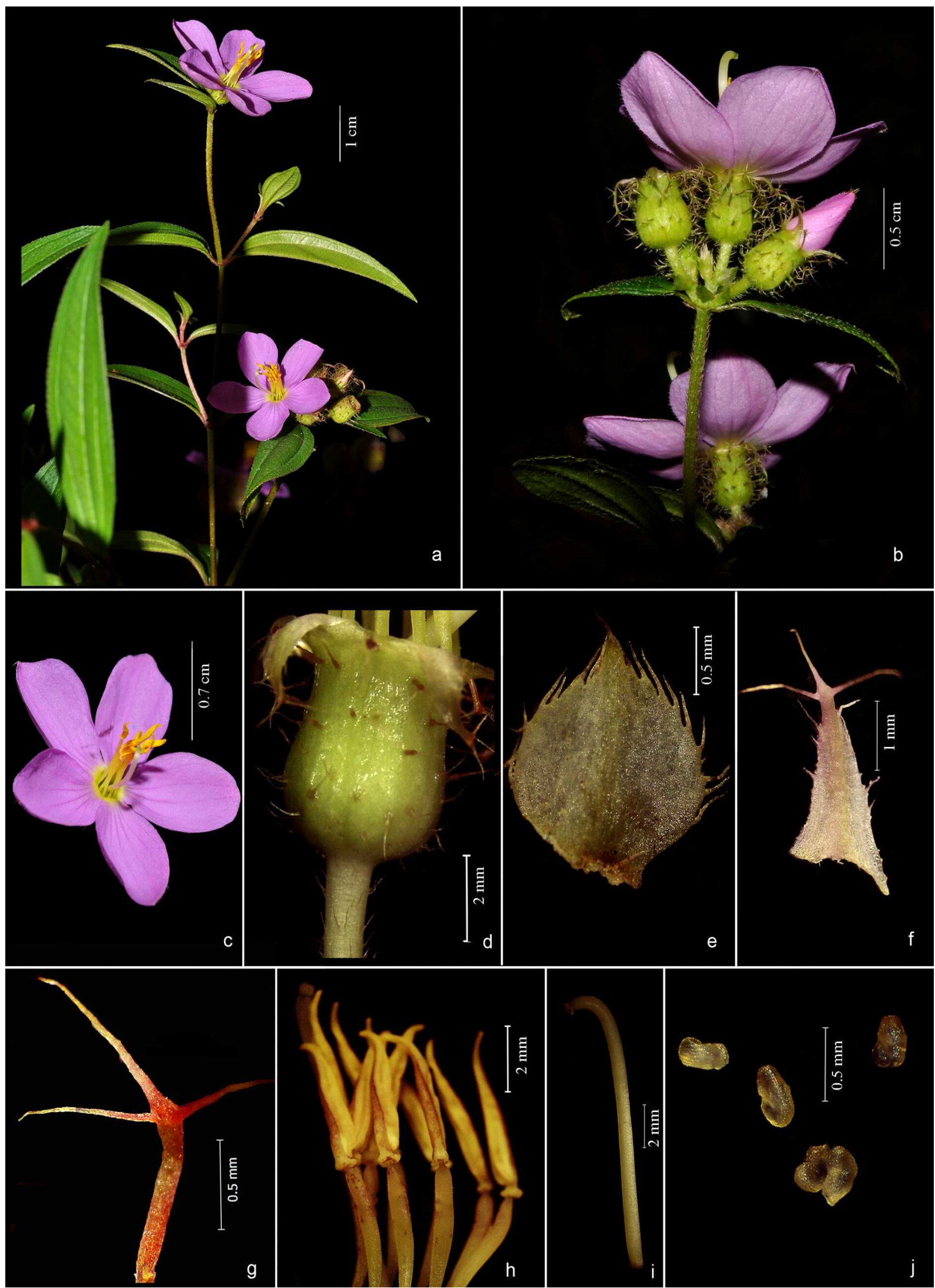

Fig. 16. Osbeckia virgata D.Don ex Wight \& Arn.: a. Habit; b. Infloresence; c. Flower; d. Hypanthium; e. Bract; f. Calyx lobe; g. Intersepalar emergence; h. Stamens; i. Style; j. Seeds. 
Perennial, erect, shrubs, $30-50 \mathrm{~cm}$ tall. Stems branched, young branches quadrangular, with appressed short hairs. Petioles 1.5-4 mm long; lamina lanceolate, $2-4.5 \times 0.5-1.5 \mathrm{~cm}$, apex acute to acuminate, base attenuate, 3-5-nerved, both sides sparsely clothed with short to very short ascending, rarely appressed bristles. Inflorescence a terminal cluster of cymes, 5-11-flowered, subtended by the upper pair of small reduced leaves. Flowers pentamerous; bracts ovate, $1-2 \times 0.5-1 \mathrm{~mm}$, green, margins ciliate, both sides glabrous: pedicels 1-2 mm. Hypanthium campanulate, 3-5 × 2-3.5 mm, sparsely clothed with emergences and bristly hairs; intersepalar emergences stalked, 0.7-2 mm long, stalk terete, narrow, glabrous, head small, with 23 spreading bristles, caducous; other emergences similar, occurring only on upper part of the hypanthium, smaller or reduced to terminal bristles, hairs patent. Sepals 5, triangular, 2-4 × 1-2 mm, narrowed into an attenuate or terete bearing stellate emergence at the apex, margins ciliate with few hairs, 1-nerved with few patent bristles dorsally, caducous. Petals 5, obovate to orbicular, 7-16 mm long, apex ciliate, pink. Stamens10 ; filaments 4-6 mm long, glabrous; anthers narrowly ovate, yellow, twisted, 3-5.5 mm long, pore oblique; connective with undulating collar. Ovary united with hypanthium for about half of its length; anther pockets extending to base of ovary; crown with a few strong bristles; style 7-11 mm long. Capsules, campanulate, 4-6 × $4.5 \mathrm{~mm}$; seeds minute, $0.1-0.3$ $\mathrm{mm}$ long, numerous, curved muricate.

Flowering \& fruiting: June-April.

Chromosome number: $n=11$ (Meenakumari \& Kuriachan, 1990).

Habitat: Commonly occurs in evergreen and shola forest margins at an elevation above $1000 \mathrm{~m}$; rarely seen below $100 \mathrm{~m}$.

Distribution: Endemic to India.

Specimens examined: INDIA, Karnataka, Chikkamagaluru district, Charmudi ghats, 17.04.1979, Saldanha \& Ramesh 786 (CAL); Kodagu district, Virajapete, 18.01.1953, K.M. Vaid 23417
(DD); Shimoga district, Agumbe, 15.05.1962, Sundara Raghavan80673(CAL);Agumbe,Begar road,13.10.1962, Sundara Raghavan 67965 (CAL); Ibid., 15.05.1962, Sundara Raghavan 80667 (CAL); Agumilar Ghats Edge, North Kanara, 09.03.1960, R.S. Rao 61264 (CAL); Ghat road to Agumbe, 13.10.1962, Sundara Raghavan 83177 (CAL); Udupi district, Someshwar, 06.02.1961, Sundara Raghavan 69368 (CAL). Kerala, Ernakulam district, Pooyamkutty, 14.02.1986, K.K.N. Nair 4304 (KFRI); Idukki district, Kalveri Mount, 08.11.1981, Ramanujan \& Mohanan 72429 (CAL); Kuttikanam, 25.09.1964, Vivekananthan 21370 (MH); Peerumedu, Kuttikanam, 15.07.1966, Vivekananthan 29325 (MH); Vallakkadavu, 11.03.1973, B.D. Sharma 84519 (MH); Kannur district, Thalappalli, 28.01.1979, V.S. Ramachandran 59273 (MH); Kollam district, Paravur, 07.08.1978, Mohanan 58401 (CAL); Kulathupuzha, 19.12.1980, Sasidharan 1438 (KFRI); Kozhikode district, Muthapanpuzha, Vellarimala, 11.07.2014, Prashob E Sibichen 5521 (DEV); Pakranthalam, 10.07.2014, Prashob \& Sibichen 5520 (DEV); Thamarassery, 29.10.1965, J.L. Ellis 25766 (CAL); Malappuram district, Nadukani, 08.1956, E. Barnes s.n. (DD); Pathanamthitta district, Konni, 30.07.1908, M. Rama Rao 0064 (DD);Ibid., 21.11.1928, V. Narayanaswami 1763 (MH); Ibid., 22.12.1957, S. Negi 25887 (DD); Manniyar River, Puthukulam, 07.07.1988, Arunkumar 640 (CAL); Ranni, 10.11.1976, Chandra Bose 49075 (MH); Ranni, 30.08.1977, N.C. Nair 507088 (MH); Thiruvananthapuram district, Bonacaud, 04.10.1973, J. Joseph 44546 (MH); Nedumangad, 11.1901, s.coll. s.n. (MH); Ponmudi, 11.09.1970, K.M. Matthew 12328 (RHT); Ibid.,17.11.1977, Mohanan 52547 (MH); Ibid., 17.01.1989, Sasidharan 5282 (KFRI); Ibid., 01.08.2014, Prashob E Sibichen 5513 (DEV); s.loc., 12.1937, N.L. Bor 83037 (DD); Thrissur district, Vazhachal, 04.11.1989, Sasidharan 5500 (KFRI); Wayanad district, Brahmagiri, 16.11.1978, V.S. Ramachandran 58761 (CAL, MH); Chandanathode, 20.12.1979, V.S. Ramachandran 65354 (CAL, MH); Chembra lake, 08.12.2014, Prashob \& Sibichen 5558 (DEV); 
Pookode lake, 04.06.2014, Prashob \& Sibichen 5509 (DEV); Ibid., 08.07.2014, Prashob \& Sibichen 5517, 5518, 5519 (DEV); Sulthan Bathery, 09.05.1965, J.L. Ellis 24009 (MH); Tirunelli, 09.02.1978, V.S. Ramachandran 53830 (MH); s.loc., 1881, Beddome s.n. (MH); s.loc., 11.1884, J.S. Gamble 172054 (CAL). Tamil Nadu, Coimbatore district, Atakatti, Anaimalai hills, 12.1911, C.E.C.Fischer 2214 (CAL); Sholayar, 20.04.1986, Sasidharan 1756 (KFRI); Nilgiri district, Conoor, 08.1883, J.S. Gamble 12307 (DD); Gudalur, 11.03.1969, D.B. Deb 31651 (CAL); Namakkal district, Kolli hills, 24.01.1968, K.M. Matthew 8478 (RHT); Ibid., 11.01.1976, K.M. Matthew E Alphonse 711 (RHT); Ibid., 13.08.1976, K.M. Matthew \& Alphonse 3154 (RHT); Ibid.,, 03.02.1978, Mohan 11587 (RHT);Kolli hills, Ariyur shola, 22.04.1980, K.M. Matthew 27636 (RHT); Ibid., 28.04.1981, K.M. Matthew 28285 (RHT); Kolli hills, Kolathukadu shola, 20.04.1978, Mohan 13192 (RHT); Kolli hills, Kolathukadu, 03.02.1978, Mohan 11624 (RHT); Kolli hills, Kuzhivalavu shola, 24.06.2001, K.M.Matthew 62736 (RHT); Kolli hills, Kasam, a pond near Sempaththu village, 08.02.1979, Perumal 21663 (RHT); Kolli hills, Selur, 17.10.1978, K.M. Matthew \& Venugopal, 18378 (RHT); Ibid., 07.02.1979, Perumal 21591 (RHT); Ibid., 21.04.1979, K.M. Matthew 22963 (RHT); Kolli hills, Solakkadu, 18.12.1979, K.M. Matthew 25185 (RHT); Kolli hills, Solakkadu, Pongal Koil shola, 22.02.1980, K.M. Matthew 26668 (RHT); Kolli hills, Kuzhivalavu top, 15.03.2002, K.M. Matthew 63342 (RHT); Kolli hills, Sothupparai, 17.03.2003, K.M. Matthew 63604 (RHT); Salem, 06.02.1979, Perumal 21663 (CAL); Salem, Mooduballi village, 15.04.1977; Ramanathapuram district, Deviar estate, 11.06.1979, Srinivasan 63515 (CAL); Sethur hills, 13.03.1981, Srinivasan 68051 (CAL).

Notes: Osbeckia virgata and O. octandra resembles in habit. Hansen (1977) also made a similar observation. But O. virgata is distinct from the latter due to the sparse vestiture of the hypanthium, thread-like intersepalar emergences having one or a few bristles, much reduced intersepalar emergences and by the deciduous sepals. Both species are found in Sri Lanka but in India there is only O. virgata.

Osbeckia wightiana (Benth.) Wight \& Arn., Prodr. Fl. Ind. Orient. 1: 323. 1834; C.B.Clarke in Hook.f., Fl. Brit. India 2: 519. 1879; Gamble, Fl. Pres. Madras 1: 493. 1919; Fyson, Fl. Ind. Hill 173. 1932; Mohanan \& Sivad., Fl. Agasthyamala 280. 2002; Anil Kumar et al., Fl. Pathanamthitta 223. 2005. O. wightiana Benth. in Wallich, Numer. List. n. 4060. 1831 (nom.nud.). O. aspera (L.) Blume var. Wightiana (Benth. ex Wight \& Arn.) Trimen, Fl. Ceylon 2: 196. 1894, syn. nov.; C.Hansen, Ginkgoana 4: 85. 1977; Vivek. in N.C.Nair \& A.N.Henry, Fl. Tamil Nadu 1: 161. 1983; Mohanan, Fl. Quilon Dist., 187. 1984; Vajr., Fl. Palghat Dist., 203. 1990; M.Mohanan \& Henry, Fl. Thiruvanthapuram 198. 1994; Sasidh. et al., Bot. Stud. Med. Pl. Kerala 37. 1996; Swarup. et al., Shola For. Kerala 59. 1998; Sasidh., Fl. Periyar Tiger Reserve 144. 1998; Sasidh., Fl. Chinnar WLS 131. 1999; Manickam et al., Fl. Tirunelveli Hills 407. 2008. Lectotype (designated by Hansen, 1977): s.loc., 1831, Wallich 4060A (K000867973 digital image!). O. koti-gueda Naudin, Ann. Sci. Nat. Bot. ser. 3, 14: 64. 1850. Lectotype (designated by Hansen, 1977): INDIA, Tamil Nadu, Nilgiris (as Nellyghery), s.d., Leschenault 41 (P02441100 digital image!)

Fig. 17

Perennial, erect, branched, woody shrubs, 1-3.5 $\mathrm{m}$ tall. Young stems quadrangular, thinly clothed with appressed or sub appressed, short bristles. Petioles 4-8 mm long; lamina ovate, 3-7 × 2-3.5 $\mathrm{cm}$, apex acute, base obtuse, 5-7-nerved, nonacrodromous, adaxial surface of leaf covers with dense silky sub-patent hairs, abaxial side with patent, flexuous hairs. Inflorescence terminal cymes, 3-7-flowered, subtended by large bracts; bracts ovate, 6-10 mm long, green, margins ciliate, densely rufous hairy. Flowers pentamerous; Pedicels 1-3 mm long, covered with thin patent hairs. Hypanthium campanulate, 7-13 × 7-12 mm, densely clothed with large emergences mixed with small hairs and bristles; intersepalar emergences stalked, 1.5-2.5 mm long, hairy, ending in a small 


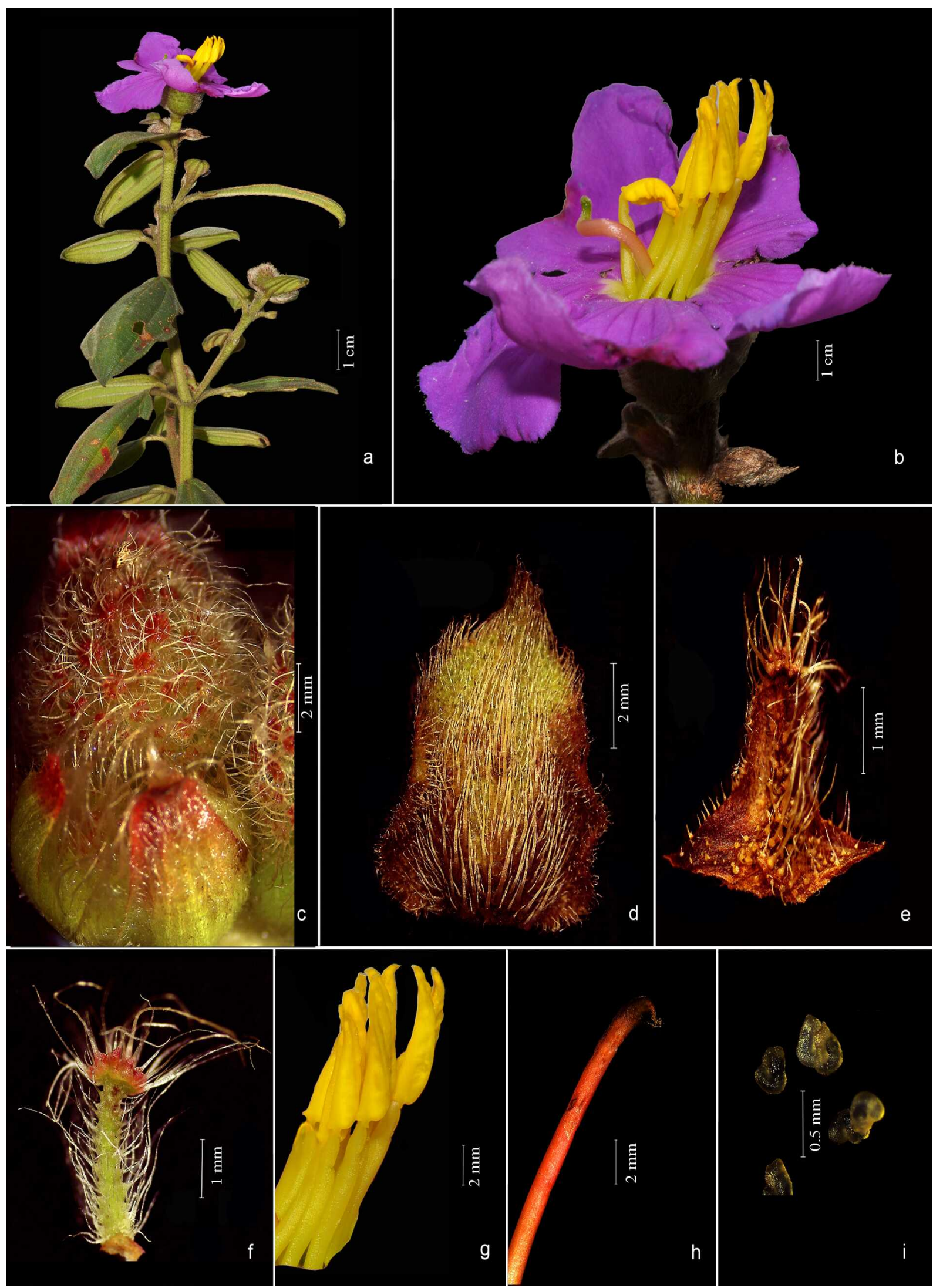

Fig. 17. Osbeckia wightiana (Benth.) Wight \& Arn.: a. Habit; b. Flower; c. Hypanthium; d. Bract; e. Calyx lobe; f. Intersepalar emergence; g. Stamens; h. Style; i. Seeds. 
tuft of patent stellate hairs; other emergences similar, mixed with small hairs and bristles. Sepals 5, subulate, $2.5-4 \times 1.3-3.5 \mathrm{~mm}$ long, sparsely hairy, margins ciliate, apex with stellate emergence, caducous. Petals 5, obovate, 15-25 × 10-20 mm long, pink to purple; margins ciliate. Stamens 10; filaments 7-12 $\mathrm{mm}$ long, glabrous; anthers narrowly oblong, yellow, 5-9 mm long, twisted, pore apical, oblique on dorsal side; connective prolonged in to a small, indistinctly lobed collar. Ovary 4-8 $\mathrm{mm}$ long, united with hypanthium; anther pockets covered with brownish appressed hairs; crown ciliate; style 1-1.5 cm long, glabrous, curved; stigma, swollen. Capsules campanulate, 5$7.5 \times 4-5.5 \mathrm{~mm}$; seeds numerous, minute, $0.1-0.2$ $\mathrm{mm}$ long, curved.

Flowering \& fruiting: July-April

Habitat: Occurs in evergreen forests at an elevation above $1000 \mathrm{~m}$, seen in association with Osbeckia virgata and $\mathrm{O}$. parvifolia.

Distribution: Endemic to South India.

Specimens examined: INDIA, Kerala, Idukki district, Kattappana, 08.11.1981, B. Ramanujan $\mathcal{E}$ C.N Mohanan 72415 (CAL); Mathikettan, 08.05.1994, V.S. Ramachandran 1778 (FRLH); Palakkad district, Poovancholai, Silent valley, 07.03.1984, N.C. Nair \& P. Bhargavan 8112 (CAL); Silent valley R.F., 22.12.1969, E. Vajravelu 33259 $(\mathrm{MH})$. Tamil Nadu, Coimbatore district, Vadakumalai, 22.12.1970, M.V. Viswanadhan 860(MH); Velliengiri hills, 21.02.1932, S.R. Raju \& Rethnavelu $215(\mathrm{MH})$; Dindigul district, Gundur shola, Kodaikanal, 10.07.1986, K.M. Matthew \& N. Rajendren 45840 (RHT); $6^{\text {th }}$ road to Kodaikanal, 09.03.1958, K. Subramanyam 5525 (MH); Kodaikanal, 13.03.1956, S.J. Pallithanam 1352 (RHT); Ibid., 14.10.2015, Prashob \& Sibichen 6012 (DEV); Kodaikanal, Perumal peak, 21.07.1984, K.M. Matthew 40344 (RHT); Leving path, Kodaikanal, 29.04.1986, K.M. Matthew \& N. Rajendren 44662 (RHT); Observatory slope, 11.08.1969, K.M. Matthew 10386 (RHT); Palamalai, 19.02.1986, K.M. Matthew 44159 (RHT); Palamalai
Machur path, 10.05.1985, K.M. Matthew \& N. Rajendren 44923 (RHT); Palani hills, 29.11.1985, K.M. Matthew \& N. Rajendran 43446 (RHT); Perumal malai, 21.05.1969, K.M. Matthew 10243 (RHT); Berijam slope, 06.08.1984, K.M. Matthew 2150 (RHT); Perumal malai, St. Joseph's farm, 07.02.1985, K.M. Matthew 40953 (RHT); Moir point slope, 04.08.1985, K.M. Matthew 41688 (RHT); Kodaikanal, Pillar Rock, 11.06.1986, C. Sundhersan 004 (CAL); Ibid., 14.09.1968, D.B. Deb 30847 (MH); Kodaikanal hills, 18.10.1919, C.K. Jacob 21171 (MH); Kanyakumari district, Mahendragiri R.F., 25.02.1983, A.N. Henry \& R. Gopalan 77157 (CAL); Upper Kothayar, 02.02.1978, A.N. Henry 53370 (MH); Sengamalai estate to Mahendragiri, 06.12.1969, B.V. Shetty 33058 (MH); Kandakki estate, Panagudi, 03.12.1969, B.V. Shetty 33015 (MH); Madurai district, Varayattu mottai, Pachakamatchi, 16.08.1990, V. Lakshmanan 91197 (MH); Nilgiris district, Kotagiri Avenue, 04.01.1957, K. Subramanyam 1888 (MH); Naduvattam, 18.01.1961, B.V. Shetty 11925 (MH); Upper Tiger shola, 19.01.1957, K.M. Sebastine 2069 (MH); Mettupalayam-Kotagiri road, 07.03.1969, D.B. Deb 31481 (MH); Kotagiri, 31.07.1970, E. Vajravellu 35184 (MH); Lamb's rock, Coonoor, 28.04.1971, N.C. Rathakrishnan 38105 (MH); Koil mattam, Elanad, 25.03.1972, G.V. Subbaro 40476 (MH); Nilgiri, Coonoor, 01.1883, J.S. Gamble 11925 (DD); Ibid., 10.1886, J.S. Gamble 21155 (MH); Ibid.,10.1889, J.S. Gamble 21437 (DD); Nilgiri, Sispara, 10.1889, J.S. Gamble 21155 (MH); Ooty, Kotagiri, 18.12.2014, Prashob \& Sibichen 5577, 5578 (DEV); Tirunelveli district, Pachayar bund, Sengaltheri, 22.12.1980, E. Vajravelu 76430 (CAL); Theni district, Periakulam, ShembaganurPeriakulam, 21.11.1985, K.M. Matthew, N. Rajendren \& A. Usha 42933 (RHT).

Notes: Trimen (1894) treatd this taxon as a variety under Osbeckia aspera. However, this taxon is recognized as a distinct species in the present treatment. Substantiating characters are summarized in the Table 3 below. 
Table. 3. Comparison of O. aspera and O. wightiana

\begin{tabular}{|l|l|l|}
\hline Character & O. aspera & O. wightiana \\
\hline Leaves & Acrodromous, 3-nerved & Non-acrodromous, 5-7-nerved \\
\hline Hypanthium & Simple hairs only & Simple hairs and emergences all over \\
\hline Bracts & Ovate to cordate, 1-5 mm long & Ovate, 6-10 mm long \\
\hline Sepals & Triangular & Subulate \\
\hline $\begin{array}{l}\text { Intersepalar } \\
\text { emergences }\end{array}$ & Sessile, ending in a tuft of hairs & $\begin{array}{l}\text { Stalked, hairy, ending in a small tuft of patent } \\
\text { stellate hairs }\end{array}$ \\
\hline Other emergences & Absent & Present, stalked with stellate head \\
\hline
\end{tabular}

Osbeckia wynaadensis C.B.Clarke in Hook.f., Fl. Brit. India 2(6): 521. 1879; Gamble, Fl. Pres. Madras 1: 493 (348). 1919; C.Hansen, Ginkgoana 4: 62. 1977; Ramach. \& V.J. Nair, Fl. Cannanore District 188. 1988; M.Mohanan \& Henry, Fl. Thiruvananthapuram 199. 1994; Sasidh. \& Sivar., Fl. Pl. Thrissur For. 190. 1996; Sasidh., Fl. Periyar Tiger Reserve 145. 1998; Sasidh., Fl. Parambikulam W.L.S. 129. 2002. Lectotype (designated by Hansen, 1977): INDIA, Tamil Nadu, Gudalur, Wayanad, $4000 \mathrm{ft}, 29.03 .1870$, C.B. Clarke 11328A (K000890001 digital image!)

Fig. 18

Perennial, erect, shrubs, $1-2.5 \mathrm{~m}$ tall. Stems profusely branched, branches thinly covered with appressed shaggy hairs. Petioles $0.5-1.5 \mathrm{~cm}$ long; Lamina ovate to elliptic, $9-20 \times 2.5-5 \mathrm{~cm}, 5-$ nerved, green, apex acute, base obtuse, upper side covered with ascending strong shaggy hairs, lower side with patent short hairs. Inflorescence terminal panicles, 9-15-flowered. Flowers pentamerous; bracts ovate, 10-14 × 4-8 mm, green, appressed hairy along the dorsal side; pedicels $0.5-1.5 \mathrm{~mm}$ long. Hypanthium campanulate, 6-9 × 3-5 mm, with a few, large scale like emergences, simple hairs absent; intersepalar emergences strongly bristled scale like, caducous; other emergences similar to those on the hypanthium. Sepals 5, triangular, 7$12 \mathrm{~mm}$ long, acute, with indistinct nerves, margins finely ciliate, both sides glabrous, caducous. Petals 5, broadly obovate, 15-19 × 8-12 mm long, purple, with ciliate apex. Stamens 10; filaments 7-11 mm long, glabrous; anthers narrow, ovate, $6-10 \mathrm{~mm}$ long, yellow, attenuate, not twisted; pore oblique on ventral side; connective with a collar having dorsal and ventral lobes. Ovary united with hypanthium; anther pockets extending to the base of ovary; crown distinct with appressed short hairs; style 12-18 mm long. Capsules campanulate, 7-8 $\times 5--6 \mathrm{~mm}$; seeds minute, numerous, muricate and curved.

Flowering \& fruiting: September-April.

Chromosome number: $n=12$ (Meenakumari \& Kuriachan, 1990).

Habitat: Usually found in large population, in swampy areas along the forest margins also occurs in open grasslands.

Distribution: Endemic to South India.

Specimens examined: INDIA, Kerala, Idukki district, Munnar, 30.04.1937, E. Barnes s.n. (DD); Palakkad district, Parambikulam Submergible area, 20.11.1962, K.M. Sebastine 15049 (CAL, MH); Wayanad district, Meenmutty, 14.02.1982, C. N. Mohann 73252 (CAL); Ibid., 27.01.15, Prashob \& Sibichen 6015, 6016, 6017 (DEV); Pookode lake, 27.01.15, Prashob \& Sibichen 6011, 6012, 6013, 6014 (DEV); Sulthan Bathery, 08.02.1964, J.L. Ellis 18623 (CAL, MH); Tirunelli, 09.02.1978, V.S. Ramachandran 53819 (CAL, MH); s.loc., s.d., E. Barnes s.n. (MH). Tamil Nadu, Dindigul district, Kodaikanal, s.d., s.coll. s.n. (RHT); Nilgiri district, Benne R.F., 20.01.1961, B.V. Shetty 11944 (MH); Ibid.,10.04.1972, K. Vivekanandhan 79662 (MH); Gudalur, Ibid., s.d., C.B. Clarke s.n. (MH); Ibid, s.d., E. Barnes s.n.(DD); Tirunelveli district, Mahendragiri, 09.1903, C.E.C. Fischer s.n. (CAL). 


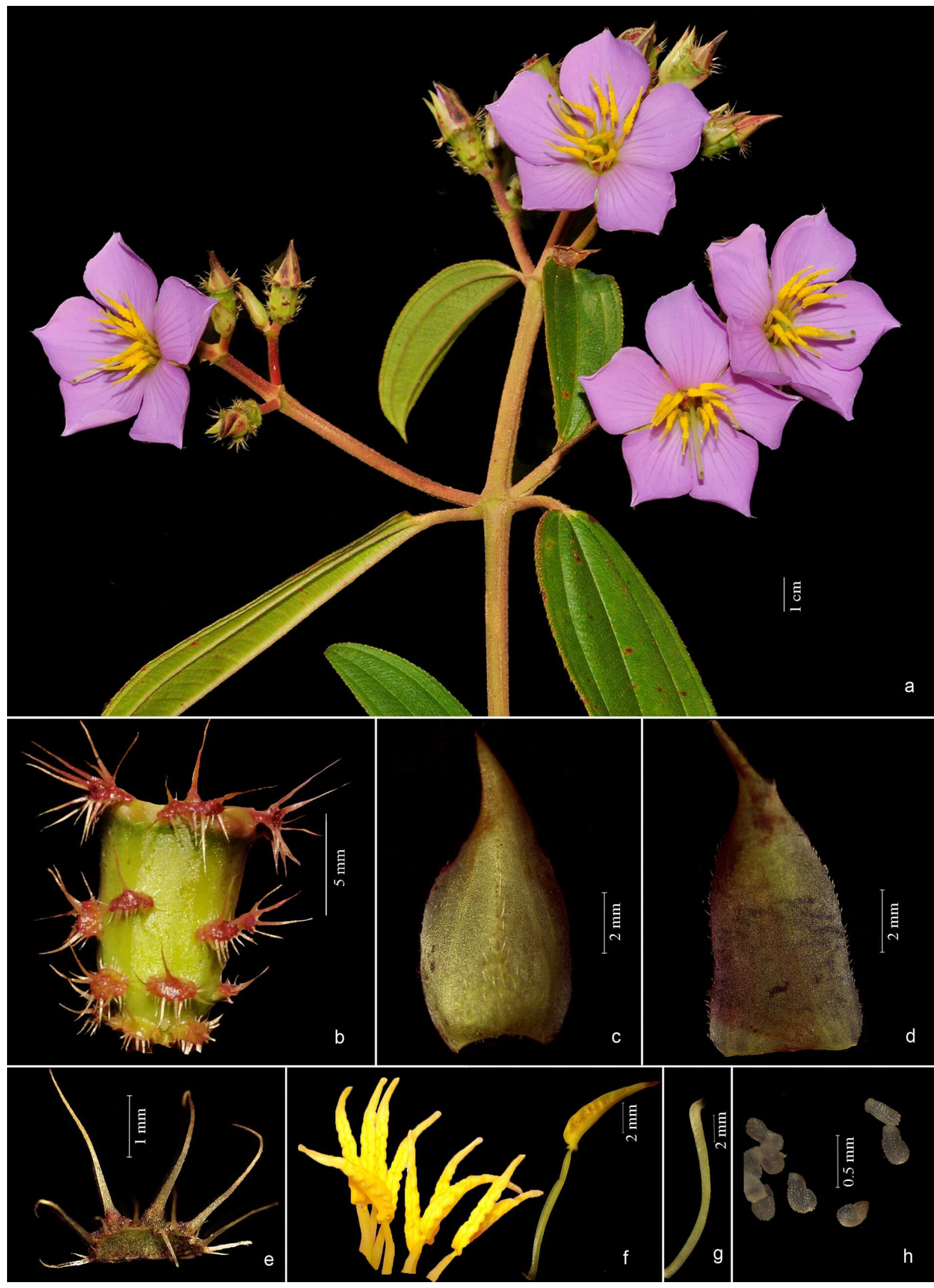

Fig. 18. Osbeckia wynaadensis C.B.Clarke: a. Habit; b. Hypanthium; c. Bract; d. Calyx lobe; e. Intersepalar emergence; f. Stamens; g. Style; h. Seeds. 
Notes: Osbeckia wynaadensis closely resembles $O$. nepalensis both in vegetative and floral characters. Both of them occur in altitudes above $700 \mathrm{~m}$. The allopatric distribution (O. wynaadensis is restricted to South India whereas O. nepalensis found only in North East) and the length of the petioles are the two major differences by which the species can be segregated. In O. wynaadensis leaves are petiolate $(0.5-1.5 \mathrm{~cm}$ long) but in O. nepalensis the leaves are sessile.

\section{Osbeckia sect. Ceramicalyx}

The section Ceramicaly $x$ was established by Triana (1871) with five species namely O. stellata, O. hispidissima, O. crinita, O. gracilis and O. rostrata and its varieties viz., var. longicollis, var. pulchella and var. ternifolia. Clarke (1879) treated all the above species except $O$. gracilis and $O$. rostrata var. ternifolia under this section. Besides these, Clarke (l.c.) also included a new variety of $O$. rostrata viz., var. marginulata. Later, Cogniaux (1891) adopted the section Ceramicalyx Triana, excluding O. gracilis. The exclusion of $O$. gracilis by subsequent authors is justifiable as the species is fairly distinct due to its pentamerous flowers and the anthers without beak. The members of the section Ceramicalyx are perennial shrubs with tetramerous flowers, urceolate hypanthium and " $\mathrm{S}$ " shaped anthers with long beak. The section is represented by 3 species and 2 varieties in India.

Osbeckia sect. Ceramicalyx Triana, Trans. Linn. Soc, London 28: 53. 1871; C.B.Clarke in Hook.f., Fl. Brit. India 2(6): 516. 1879; Cogn. in A.DC. \& C.DC., Monogr. Phan. 7: 322: 1891. Type: Ceramicalyx stellatus Buch.-Ham. ex D.Don (= Osbeckia stellata Buch.-Ham. ex D.Don).

Oxyrhinae subsect. Longicolles Naudin, Ann. Sci. Nat. Bot. ser. 3. 14: 74. 1850.

Perennial, erect, branched shrubs. Flowers tetramerous; hypanthium urceolate with stellate emergences and hairs; anthers 'S' shaped with long beak.

Distribution: Distributed mainly along wet tropical and subtropical Asia including Indo China, Nepal, and Bhutan.

\section{Key to the species of sect. Ceramicalyx}

1a. Plant glabrous; branchlets distinctly winged; hypanthium without emergences and hairs; intersepalar emergences absent ....... O. nayarii

1b. Plant hairy; branchlets not distinctly winged; hypanthium with emergences and hairs; intersepalar emergences present 2

2a. Intersepalar emergences having terete stalk with lateral hairs and stellate head O. stellata

2b. Intersepalar emergences linear without lateral hairs and stellate head.... O. rostrata

Osbeckia nayarii G.S.Giri, J. Econ. Taxon. Bot. 4(2): 609. 1983. Type: INDIA, Meghalaya, Khasia, 2000 ft., 14.09.1886, C.B. Clarke 44776A (holo CAL0000015810!).

Melastoma pulchella Roxb., Fl. Ind., ed. 2, 2: 403. 1832. Type: INDIA, s.loc., 1814, Roxburgh s.n. (holo BM000944362 digital image!).

Ceramicalyx pulchellus Blume, Mus. Bot. 1(4): 50. 1849. Type: BANGLADESH, Chittagong, s.d., Wallich 4059B (holo K000639584 digital image!).

O. pulchella Benth. ex Wall., Numer. List Pl. 143, no. 4059. 1831. nom. nud. non Benth. ex Naudin, 1850.

Fig. 19

Perennial, erect, shrubs, 1-1.5 m tall, with straggling branches. Stems and branches strongly quadrangular, winged at angles: wings thick, more or less fleshy, glabrous throughout. Petioles 5-10 mm long; lamina elliptic to lanceolate, 10-15 × 3$6 \mathrm{~cm}, 5-7-$ nerved, apex acute to acuminate, base cuneate, glabrous, dark green. Inflorescence terminal condensed panicles, 5-12-flowered. Flowers tetramerous; pedicels 3-7 mm long; bracts green, ovate, $8-13 \times 2-6 \mathrm{~mm}$ long, glabrous on either side, caducous. Hypanthium urceolate, 12$14 \times 4-6.5 \mathrm{~mm}$, glabrous; intersepalar emergences absent. Sepals 4, narrowly ovate with prominent midrib, $10-13 \times 2.5-3.5 \mathrm{~mm}$, glabrous, not persistant in fruit. Petals 4, ovate to orbicular, 16-20 


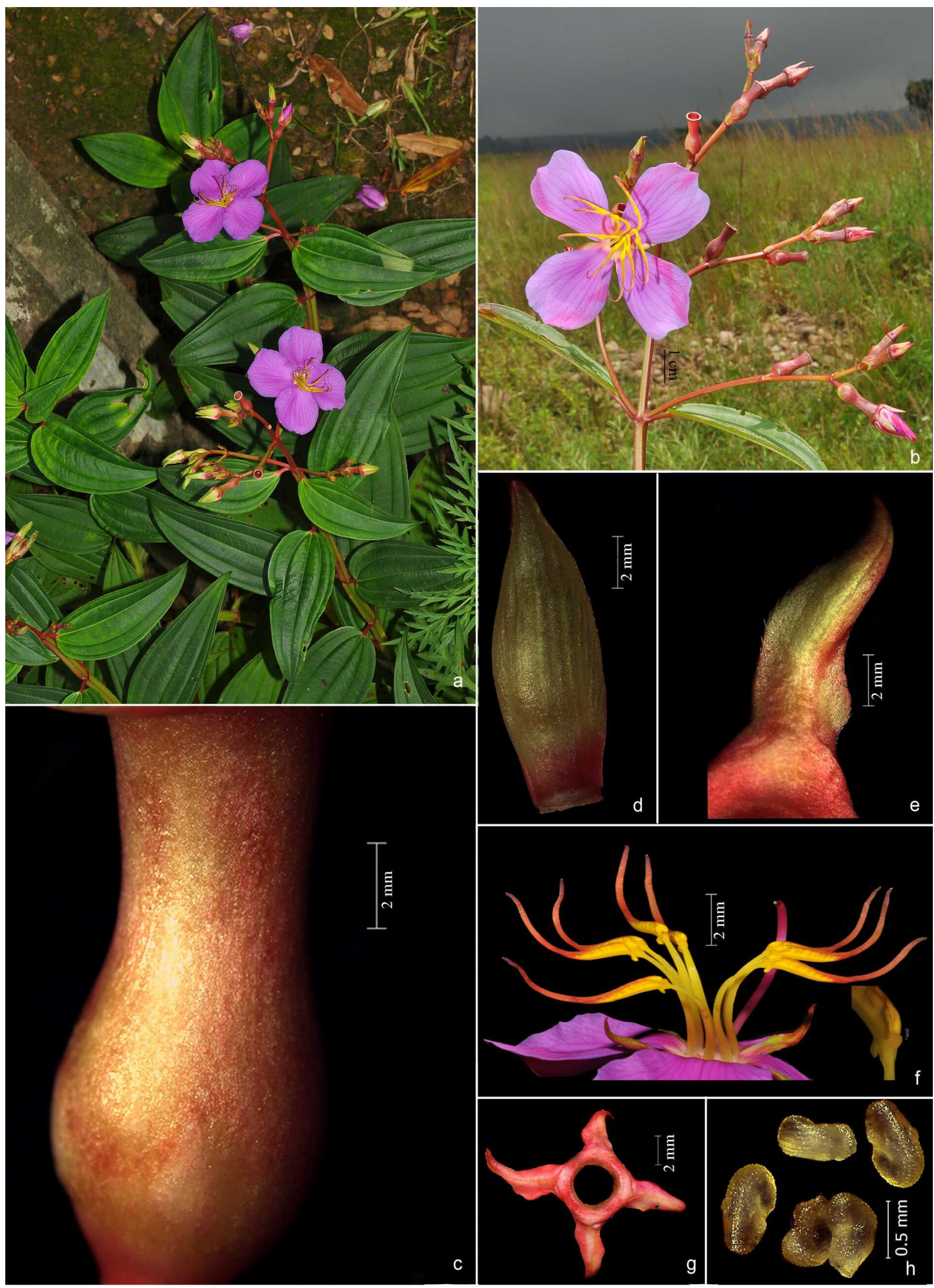

Fig. 19. Osbeckia nayarii G.S.Giri: a. Habit; b. Flower; c. Hypanthium; d. Bract; e. Calyx lobe; f. Stamens; g. Calyx lobes without intersepalar emergence; $\boldsymbol{h}$. Seeds. 
$\times 10-14 \mathrm{~mm}$, pink to purple. Stamens 8, filaments 2-4 cm long, glabrous; anthers ' $\mathrm{S}$ ' shaped, 2-4.5 $\mathrm{cm}$ long, attenuate into a narrow, tubular beak (4$8 \mathrm{~mm}$ long), yellow, glabrous, pore apical, connective prolonged in to a small, indistinctly lobed collar. Ovary 12-14 mm long, 4-locular, united with hypanthium; anther pockets and crown covered with appressed hairs; style 2.5-4 mm long, glabrous, curved; stigma slightly swollen. Capsules urceolate, 12-14 × 4-6.5 mm, glabrous with a long neck; seeds numerous, minute, 0.1-0.2 mm long, curved and muricate.

Flowering \& fruiting: July-November.

Habitat: Found in open grasslands above $1000 \mathrm{~m}$.

Distribution: India.

Specimens examined: INDIA, Assam, Golpara district, Golpara, 1914, U. Kanjilal 6914 (CAL). Meghalaya, East Khasia hills district, Pynursla, 1755 m, 15.09.2015, Prashob \& Sibichen 6112 (DEV); Ri-Bhoi district, Nongpoh, $987 \mathrm{~m}$, 06.08.2014, K.M. Manudev E Prashob 57412 (DEV).

Tamil Nadu, Coimbatore district, Valparai, Sholayar dam, 1225 m, 03.10.2015, Prashob \& Sibichen 6018 (DEV). Kerala, Kozhikode, St. Joseph's College Botanical garden (cultivated), 55 m, 06.10.2015, Sibichen E Prashob 1620 (DEV).

Notes: Roxburgh (1832) described Melastoma pulchella in his Flora of India based on specimens collected from Chittagong. Prior to the publication of Roxburgh, Wallich (1831) included this name in his catalogue as a species of Osbeckia viz., O. pulchella Benth., treating Roxburgh's name (Melastoma pulchella) as its basionym. However, Wallich's name is not valid as it is not accompanied by a description, diagnosis, or reference to any published diagnosis. Later Blume (1849) adopted Wallich's specific epithet and placed this taxon under the genus Ceramicalyx and validly published as $C$. pulchellus. The specific epithet "pulchella" cannot be validly combined with the genus Osbeckia as it was preoccupied for another taxon, O. pulchella Benth. ex Naudin, (=O. rostrata D.Don). Therefore, the present valid name for the taxon is O. nayarii G.S.Giri.

Osbeckia rostrata D.Don, Prodr. Fl. Nep. 221. 1825; N.P.Balakr, Fl. Jowai \& Vicinity 205. 141. 1983. O. stellata Buch.-Ham. ex D. Don var. rostrata (D.Don) C.Hansen, Ginkgoana 4: 28. 1977, syn. nov. Type: NEPAL, Narainhetty, 11.08.1802, Hamilton s.n. (holo BM000521786 digital image!).

O. recalva Bedd. ex Gamble, Fl. Pres. Madras 1: 494. 1919

O. ternifolia D.Don, Prodr. Fl. Nep. 221. 1825. Ceramicalyx ternifolius Blume, Mus. Bot. 1(4): 50. 1849. O. rostrata D.Don, var. ternifolia (D.Don) Triana, Trans. Linn. Soc., London 28: 54. 1872. Type: NEPAL, s.d., Hamilton s.n. (holo BM000521785 digital image!).

O. quaterna Buch.-Ham. ex D.Don Prodr. Fl. Nep. 221. 1825, pro syn.

Ceramicalyx calvus Blume, Mus. Bot. 1(4): 50. 1849. Type: s.loc., s.d., Wallich s.n. (holo G00310438 digital image!).

O. pulchella Benth. ex Naudin, Ann. Sci. Nat. Bot., ser. 3, 14: 73. 1850. O. rostrata D.Don var. pulchella Triana, Trans. Linn. Soc., London 29: 54. 1872, nom. nud. Type: INDIA, Meghalaya, Khasia, s.d., Griffith s.n. (holo P02441144 digital image!).

O. rostrata D.Don, var. longicollis Triana, Trans. Linn. Soc., London 28: 54. 1872, nom. nud.

O. chinensis L. var. lancifolia Kuntze, Rev. Gen. Pl. 1: 247. 1891. Type: CHINA, s.loc., s.d., Kuntze 7209 (holo NYBG00229578 digital image!).

O. rostrata D.Don var. sexangulata Haines, Bot. Bihar \& Orissa 370. 1922. Type: INDIA, Jarkhand, Chota Nagpur, wet places, $3000 \mathrm{ft}$, s.coll. s.n. (holo CAL!).

O. arunkumarensis M.P.Nayar \& G.S.Giri, J. Bombay Nat. Hist. Soc. 81(2): 436. 1984. syn. nov. Type: INDIA, Sikkim, East Himalaya, s.d., Griffith 2248 (holo CAL0000016807!).

O. campestris Buch.-Ham. in Wall., Numer. List 143, no. 4063. 1831, nom. nud. 


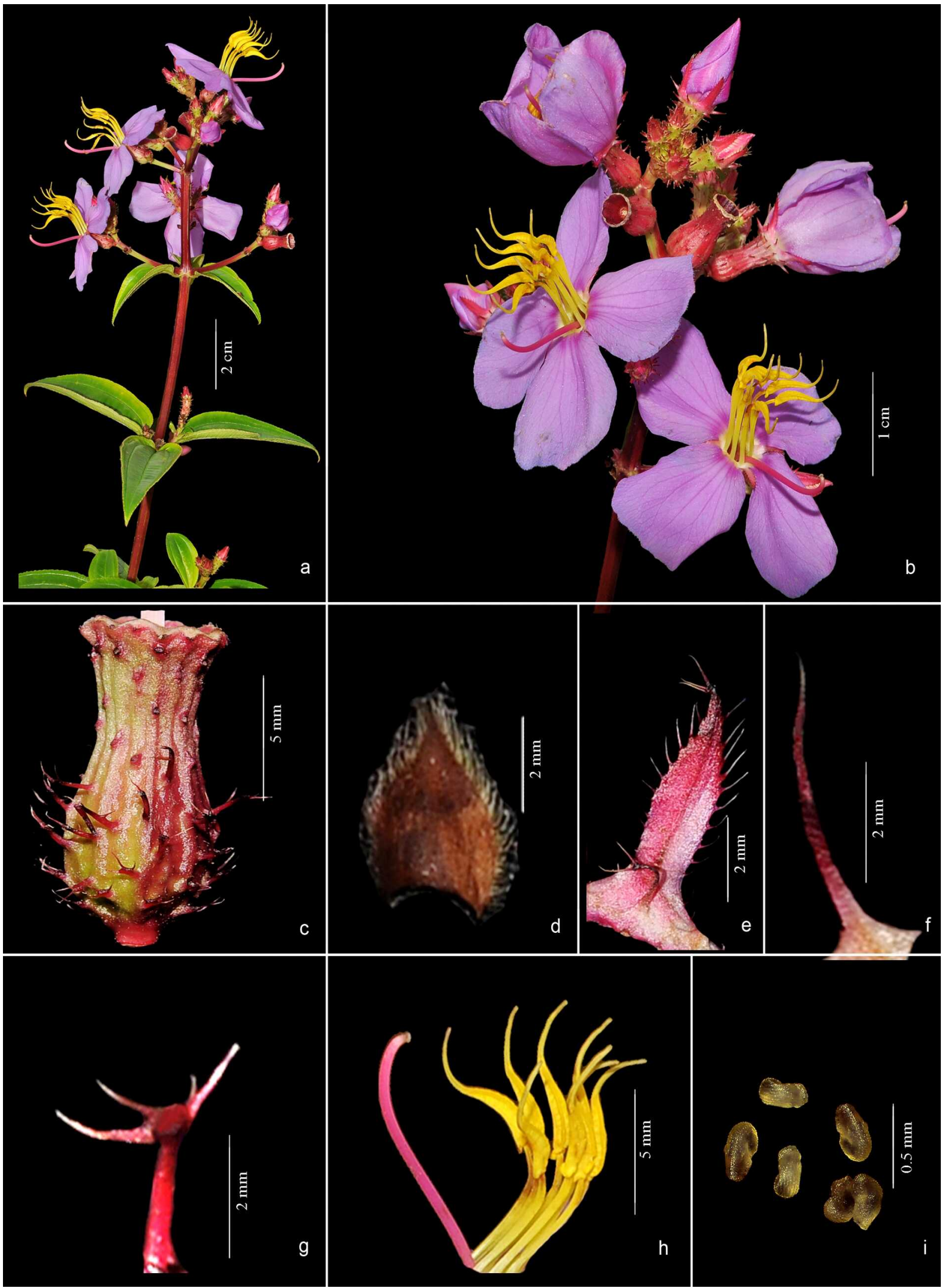

Fig. 20. Osbeckia rostrata D.Don: a. Habit; b. Flower; c. Hypanthium; d. Bract; e. Calyx lobe; f. Intersepalar emergence; g. Other emergence; h. Stamens; i. Seeds. 
O. longicollis Benth. in Wall., Numer. List 143, no. 4065. 1831, nom. nud.

Fig. 20

Perennial, erect, shrubs, 0.5-2 m tall. Stems branched; young branches quadrangular, with short, rigid, appressed hairs, sometimes occurring only at nodes. Petioles 3-8 mm long, upper leaves often sessile; lamina ovate to lanceolate, $5-17 \times$ 2-6 cm, 5-nerved, green, apex acute to attenuate, base obtuse, both sides densely clothed with patent hairs. Inflorescence a terminal panicle, 6-12flowered. Flowers tetramerous; pedicels $0.5-3 \mathrm{~mm}$ long; bracts ovate to broadly ovate, 4-8 $\mathrm{mm}$ long, glabrous, with a few to many appressed hairs dorsally. Hypanthium urceolate, 7-13 × 2.5-4 mm, usually longer than ovary, covered with emergences having a few to several patent hairs on the stalk; intersepalar emergences linear without lateral hairs and stellate head, caducous; other emergences similar. Sepals 4, lanceolate, 6-12 $\times$ 1.5-3 mm long, ciliate and with few to many patent hairs dorsally, caducous. Petals obovate to broadly obovate, $13-18 \times 10-15 \mathrm{~mm}$, glabrous pinkto purple. Stamens 8; filaments 7-10 mm long; anthers 7-13 mm, 'S' shaped, attenuate into a narrow long beak, yellow; pore oblique on ventral side; connective prolonged into a slightly undulating collar with large dorsal lobes. Ovary united with hypanthium for about two thirds of its length, anther pockets extending to the base of the ovary; remaining part glabrous or often thinly clothed with short appressed hairs, crown present; style 15-25 mm long, glabrous. Capsules urceolate, $7-11 \times 2-6 \mathrm{~mm}$, covered with emergences, neck widens towards rim, emergences fall off at maturity; seeds minute, $0.1-0.2 \mathrm{~mm}$ long, numerous, curved.

Flowering \& fruiting: August-October.

Chromosome number: $n=10$ (Meenakumari \& Kuriachan, 1990).

Habitat: Occurs in evergreen forest of North East Himalaya at an elevation above $500 \mathrm{~m}$. in association with O. stellata var. stellata.

Distribution: India.
Specimens examined: INDIA, Andhra Pradesh, Visakhapatnam district, Visakhapatnam, Sunkerimetta. 21.09.1961, N.P. Balakrishnan 730 (CAL). Assam, East Karbi Anglong district, Phulai, 28.06.1963, D.B. Deb 35403, 35404 (ASSAM); Goalpara district, Dudhnai, Rangjuli East, 25.06.1964, A.S. Rao 39113 (ASSAM); Nowgong, Sonaikushi R.F., Tapatala, 20.08.1964, N.P. Balakrishnan 39300 (CAL); s.loc, 31.07.1908, W.G. Craib 30 (CAL); s.loc., 31.07.1908, W.G. Craib 30 (CAL). Manipur, Bishnupur district, Bishnupur, 11.1907, A. Meebold 171996 (CAL); Tamenglong district, Mala naga-Cachar hills, 05.1882, G. Watt 171995 (CAL); Naga-Cachar hills, 05.1881, G. Watt 7278 (CAL); Ukgrul district, Yhirighak, 11.1907, A. Meebold 5724 (CAL); s.loc., 11.1907, Meebold 5980 (CAL). Meghalaya, East Khasi hills district, Mawlynnong, 12.09.2015, Prashob \& Sibichen 6130 (DEV); Mawsmai, 21.09.1967, H. Deka 37719 (ASSAM); Shillong, 24.06.1938, S.R. Sharma 18392 (ASSAM); Garo hills district, s.loc., s.d., N.E. Parrey 1929 (CAL); Jaintia Hills district, Jarain garden, 19.09.2015, Prashob \& Sibichen 6145 (DEV); Jarain to Syndi, 17.08.1968, N.P. Balakrishnan 46164 (ASSAM); Jowai, 19.09.2015, Prashob \& Sibichen 6152 (DEV); Nongtalang village, 19.09.2015, Prashob E Sibichen 6148 (DEV); s.loc., 09.08.1840, H.F. Mooney 1299 (ASSAM); s.loc., 26.10.1956, P.O. Khant 5158 (ASSAM). Mizoram, Aizawl district, Dampa Tiger reserve, Teirei, 21.09.2006, B.K. Sinha \& N. Odyuo 112739 (ASSAM). Tripura, Bajapara, 29.08.1957, D. B. Deb, 1115 (CAL); Charilam, 04.06.1959, D. bfer 1763 (CAL). West Bengal, Darjeeling district, Darjeeling, 26.16.1876, J.S. Gamble 1730 (DD); Sukhia Pokhri, 26.10.1876, J.S. Gamble 1735 (DD).

Notes: Hansen (1977) treated O. rostrata as a variety of O. stellata. But it differs from the latter by linear lanceolate to falcate intersepalar emergences and fleshy stellate emergences on hypanthium hence it is treated here as a distinct species. Nayar and Giri (1984) described O. arunkumarensis from the herbarium collections of Griffith from Sikkim. The diagnostic characters of $O$. arunkumarensis such as winged branches, subsessile leaves, intersepalar 
emergences terminated by a bristle are overlapping with $O$. rostrata, and hence treated here as conspecific.

Osbeckia stellata Buch.-Ham. ex D.Don., Bot. Reg. 8: t. 674. 1822; Osmaston, Fl. Kumaon 247. 1927; C.Hansen, Ginkgoana 4: 23. 1977; M.B.Raizada \& H.O.Saxena, Fl. Mussoorie 247. 1978; N.P.Balakr. Fl. Jowai \& Vicinity 206. 1983; R.K.Gupta, Fl. Nainitalensis 134. 1993; Hajra \& Verma, Fl. Sikkim1996; D.S.Dhaliwal \& M.Sharma, Fl. Kullu district 290. 1999; R.D.Gaur, Fl. Garhwal 317. 1999; Pull., D.M.Rao \& K.S.Ramamurthy, Fl. Eastern Ghats 57. 2002. Ceramicalyx stellatus Buch.Ham. ex D.Don Blume, Mus. Bot. 1(4): 50. 1849. Lectotype (designated by Hansen, 1977): NEPAL, Narainhetty, 10.08.1802, Hamilton s.n. (BM000944402 digital image!).

O. marginulata Wall., Numer. List 143, no. 4064. 1831, nom. nud. O. rostrata D.Don var. marginulata C.B.Clarke in Hook.f., Fl. Brit. India 2: 518. 1879. O. stellata Buch.-Ham. ex D.Don var. marginulata (C.B.Clarke) C.Hansen, Ginkgoana 4: 27. 1977. syn. nov. Type: MYANMAR, Tong Dong, s.d.,Wallich 4064 (holo K001038066 digital image!).

O. racemosa Craib, Bull. Misc. Inform. Kew 1911(1): 51. 1911. Type: THAILAND, Chiengami, Doi-Sootep, s.d., Kerr 836 (holo K000867939 digital image!)

O. hildebrandii Stapf, Bull. Misc. Inform. Kew 6: 269. 1911. Type: MYANMAR, Shan States, Toungyi, Cult. Hort. Kew, 1901, Hildebrand 59999 (holo K000890027 digital image!).

O. speciosa Naudin, Ann. Sci. Nat., Bot. Sér. 3, 14: 73. 1850. Type: INDIA, Mossouri, s. d., Jacquemont s.n. (holo P02441142 digital image!).

O. darjeelingensis G.S.Giri \& M.P.Nayar, Bull. Bot. Surv. India 25(1-4): 241.1985. (syn. nov.) Type: INDIA, West Bengal, Darjeeling, Jhenaikuri, 12.1879, Gamble 7597 (holo CAL0000016808!).

Fig. 21

Perennial, erect, branched, shrubs, 0.50-2.5 m tall. Stems branched, young branches quadrangular, with appressed, short, crooked, rigid hairs. Petioles 4-10 mm long; lamina green, lanceolate, 6-20 × 2-9 cm, 5-7-nerved; apex acuminate, base obtuse to attenuate, densely clothed with patent hairs on either side. Inflorescence terminal, panicled cyme, 5-9-flowered. Flowers tetramerous; pedicels 0.5-3 mm long; bracts ovate to broadly ovate, $7 \times 12$ $\mathrm{mm}$, ciliate, with few to many appressed hairs dorsally. Hypanthium urceolate, 9-20 × 3-7 mm, longer than ovary, covered with emergences and bristles inter mixed with patent or appressed hairs; intersepalar emergences 5-9 $\mathrm{mm}$ long, with a terete stalk bearing lateral hairs and terminating in a head of stellate hairs, caducous; other emergences smaller in lower portion of hypanthium. Sepals 4, triangular to lanceolate, $8-17 \times 2-4 \mathrm{~mm}$ long, ciliate and with a few to many patent hairs dorsally, caducous. Petals 4, obovate to broadly obovate, $18-25 \times 22-30 \mathrm{~mm}$, glabrous, margin ciliate, pale pinkto purple, Stamens 8; filaments 7-18 mm long; anthers 'S' shaped, yellow, 11-21 mm long, attenuate into a narrow, tubular beak (5-7 mm long), not twisted; pore oblique on ventral side; connective prolonged into slightly undulating collar with large dorsal lobe. Ovary united with hypanthium for about two thirdof its length, anther pockets extending to the base of the ovary; remaining part glabrous or thinly clothed with short appressed hairs: style 15-25 mm long, glabrous. Capsules urceolate, 11-19 × 4.5-10 $\mathrm{mm}$, neck widens towards rim, densely covered with emergences and hairs which often fall off when mature; seeds minute, 0.1-0.2 mm long, numerous, curved, muricate.

\section{Key to the varieties}

1a. Stalk of intersepalar emergences glabrous ..... O. stellata var. hispidissima

1b. Stalk of intersepalar emergences hairy ......... 2

2a. Hypanthium $(>1.5 \mathrm{~cm})$, sepals subulate .. O. stellata var. stellata

2b. Hypanthium $(<1 \mathrm{~cm})$, sepals narrowly triangular O. stellata var. crinita 


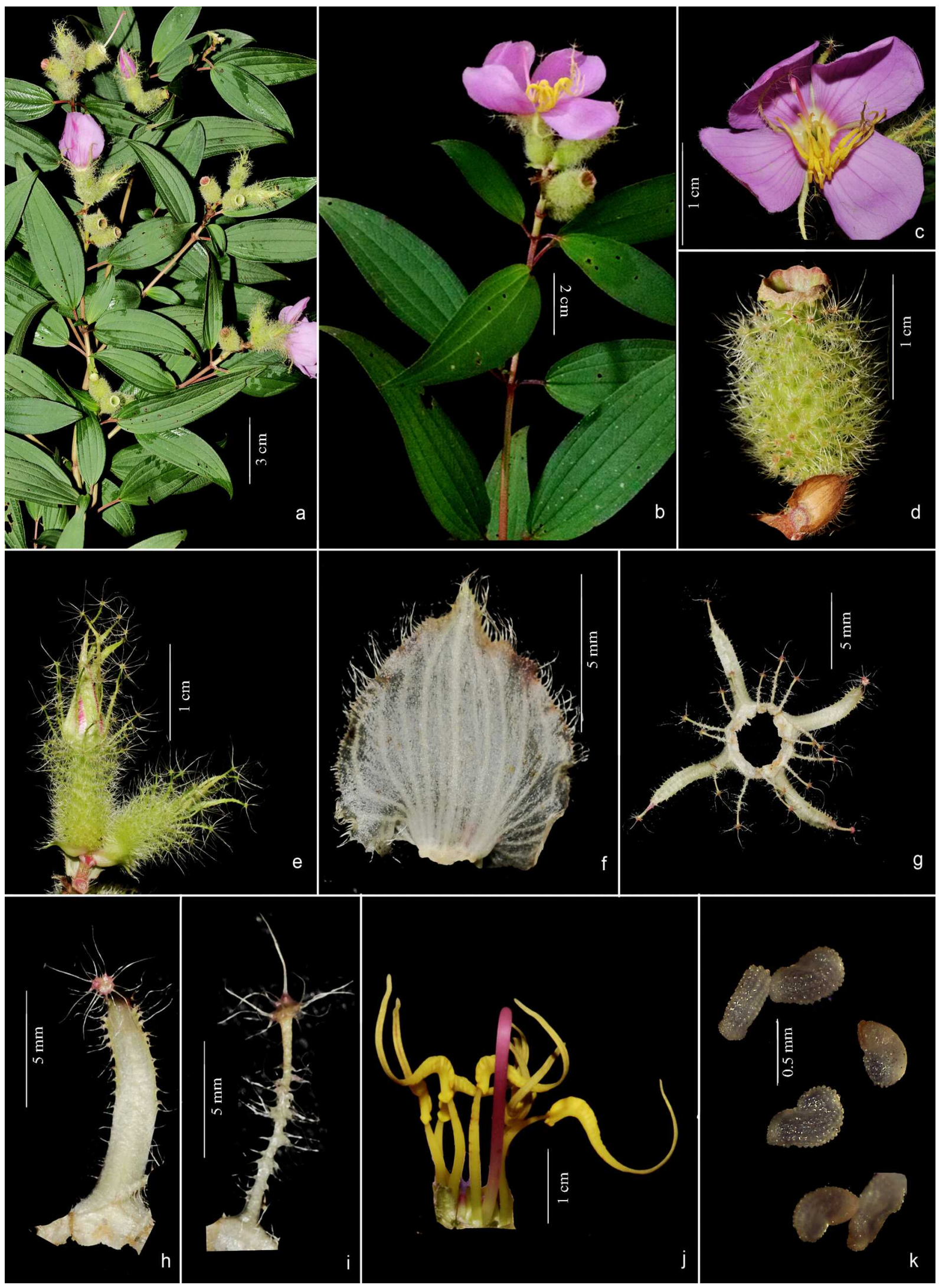

Fig. 21. Osbeckia stellata Buch.-Ham. ex D.Don var. stellata: a \& b. Habit; c. Flower; d \& e. Hypanthium; f. Bract; g. Calyx lobes with intersepalar emergences $\mathbf{h}$. Calyx lobe; i. Intersepalar emergence; j. Stamens; $\mathbf{k}$. Seeds. 
Osbeckia stellata Buch.-Ham. ex D.Don. var. stellata

Flowering \& fruiting: August-March.

Chromosome number: $n=10$ (Meenakumari \& Kuriachan, 1990)

Habitat: Seen in cluster on the margins of evergreen forests at an elevation above $500 \mathrm{~m}$. The associated species are O. stellata var. crinita and O. stellata var. hispidissima.

\section{Distribution: India.}

Specimens examined: INDIA, Arunachal Pradesh, East Siang district, Yambung Camp, 07.01.1911, I.H. Burkill s.n. (CAL); Lohith district, Khamti village, 09.09.1969, A.S. Rao 47760 (CAL, ASSAM); Khopa village, 26.09.1969, A.S. Rao 48128 (CAL, ASSAM); Lohith, 24.11.1969, J. Joseph 48314 (CAL); Lohith- Payanava, 17.11.1957, R.S. Rao 10674 (CAL); Siang F.D., Tuting, 06.11.1958, R.S. Rao 17366 (CAL, ASSAM). Ibid., 03.04.1958, G.K. Murty 13126 (CAL, ASSAM); Lower Subansiri district, Subansari F.D., Kherbavei, 28.09.1959, G. Panigrahi 19732 (CAL); Subansiri-Klerbari, 28.09.1954, G. Panigrahi 19732 (CAL); Ziro, 01.10.1959, G. Panigrahi 19900 (CAL). Himachal Pradesh, Kangra district, Baslahar, 26.06.1923, R.N. Rarker s.n. (DD); Ibid., 13.06.1928, R.N. Parker 3013(DD); Ibid., 07.10.1940, H.F. Mooney 87460 (DD); Ibid., 13.10.1950, M.B. Raizada 19962 (DD); Ibid., 12.09.1969, C.N. Nair 30666 (CAL, BSD); Ibid., 12.09.1969, C.N. Nair 30666 (CAL, BSD); Palampur, 26.09.1896, G.A. Gammie 18722 (DD); Upper Baslahar, 07.1950, S.P. Selhi 20360 (DD); Kullu district, Shaman, 20.08.1977, S.K. Murti E R. Prasad 61917 (BSD); Shimla district, Fifth Water fall, Yamunotri, 20.06.1953, S.N. Mitra 9430 (CAL); North West Himalaya, 10.1891, J.H. Lace s.n. (DD). Manipur, Bishnupur district, Limatak, 27.08.1887, George Watt 7175 (CAL). Sikkim, East Sikkim district, Machong, Chota pathing, 09.07.1991, N.R. Mandal \& P. Singh 13583 (BSHC); Rangpo, 14.10.1949, K. Biswas 9848 (CAL); Rumtek, 06.08.1981, B. Krishna 1629 (BSHC); Singtam, 12.05.1967, N.C. Mazudia \& R. M Dutta 210 (CAL);
North Sikkim district, Chungthang to Lachen, 16.08.1989, N.R. Mandal, 10447 (BSHC); Mangan, 15.11.1901, Dr. Prain s.n. (CAL); Mangan, Rang Rang bridge, 17.09.2016, Prashob, Anilkumar \& Sibichen 6616 (DEV); near Tista bridge, 26.07.1986, D.C.S. Raju \& S. Singh 6312 (BSHC); South Sikkim district, Mogipokri, s.d., R.C. Srivastava 14530 (BSHC); Namchi forest, 10.08.1992, R.C. Srivastava 10989 (BSHC); Rateypani Suntale, 11.09.1981, B. Krishna 1752 (CAL, BSHC); West Sikkim district, Dentam, 29.08.1994, P. Singh 16340 (BSHC); Dherap, 14.09.2016, Prashob, Anilkumar \& Sibichen 6608 (DEV); Geyzing, 13.09.2016, Prashob, Anilkumar \& Sibichen 6601 (DEV); Pelling, 13.09.2016, Prashob, Anilkumar \& Sibichen 6602 (DEV); Ibid., 14.09.2016, Prashob, Anilkumar \& Sibichen 6610 (DEV); Pelling to Yuksom, 16.08.1985, D.C.S. Raju 4304 (BSHC). Uttar Pradesh, Saharanpur district, Sharanpur, 09.1898, Rao s.n. (CAL); s.loc., 04.05.1916, R.S. Hole 252 (CAL). Uttarakhand, Almora district, Dinapani, 12.10.1975, J.N. Vohra 57813 (BSD); Debra, 13.10.1975, B.M. Wadhwa 57694 (BSD); Dwarahat, 05.10.1975, B.M. Wadhwa 57392 (BSD); Bageshwar district, Kapkot, 19.09.1957, T.A. Rao 4187 (BSD); Chamoli district, Gopeswar to Mandal, 29.08.1978, Panigrahi \& Wadhwa 65596 (BSD); Narayan bagarah, 07.09.1985, H.J. Chawdhary 78256 (BSD); Sitel, 09.1993, s.coll. 77199 (BSD); Sutal-Sitel, 09.1993, s.coll. 87956 (BSD); Dehradun district, Dehradun-Sahasradhara, 06.09.1963, S.K. Malhothra 29271 (CAL, BSD); Ibid., 14.09.1962, S.K. Malhothra 23788 (CAL, BSD); Mussori, 08.1895, P.W. Mackinnon s.n.(CAL); Ibid., 1859, G. King s.n. (CAL); Ibid., 06.09.1960, Hari Om Saxena s.n. (DD); Thangaon, s.d., Bipin Balodi \& Megh Singh 73076 (BSD); Garhwal district, Garhwal, 10.09.1972, B.D. Naithani 3677 (DD); GarhwalDodital, 09.1995, Raj K. Gupta 933 (CAL); Garhwal-Gauri kund, 14.08.1968, M.A. Rao 38752 (CAL, BSD); Garhwal-Mondal, 01.10.1970, B.D. Naithani 41974 (CAL, BSD); GarhwalVishnuprayag-Govind Ghats, 14.08.1963, U.C. Bhattacharya 29324 (CAL, BSD); Kumaon, Dalfia, 01.09.1973, C.M. Arora 53231 (DD); Kumaon- 
Girgaon, 29.08.1969, Pant \& Naithani 38565 (CAL, BSD); Kumaon-Munsiari, Pithoragarh, 30.08.1900, Juayal 24438 (CAL, DD); Ibid., 12.06.1960, T.A. Rao 11788 (CAL, BSD, DD); Ibid., 10.06.1960, T.A. Rao 11672 (CAL, BSD); Ibid., 12.10.1962, Surendra Singh 23622 (CAL, BSD); Ibid., 05.05.1965, C.N. Nair 35674 (CAL, BSD, DD); Ibid., Askol, 31.01.1971, C.M. Arora 45452 (BSD); Kumaon-Tavaghat, 10.06.1960, s.coll, s.n. (CAL); Naitwar, Tehri-Garhwal, 21.09.1955, K.C. Sahni 21513 (DD); Napainkoli, 22.09.1958, M.A. Rao 6468 (BSD); Rampur Garhwal, 20.10.1938, Kirt Ram 8969 (DD); Ringalgarh, Tehri-Garhwal, 26.09.1954, K.C. Sahni 21513 (DD); Tehri, Budhalcedar, 02.10.1974, M.V. Viswanathan 55224 (BSD); Tehri, Ghuttu, 18.08.1978, A.K. Goel 64588 (BSD); Pauri district, Balakhal, 09.08.1976, R.P. Srivastav 57078 (BSD); Khirsu, 18.09.1975, A.S. Rao 56332 (BSD); Khirsu road, 19.08.1978, G. Panigrahi 65000 (BSD, DD); s.loc., 15.05.1989, P.S. Karki 68633 (BSD); Pithoragarh district, Didihat, 01.10.1970, C.M. Arora 41366 (BSD); Didihat, Narayan nagar, 30.09.1975, C.M. Arora E P. Prasad 56631 (BSD); Dhanliganga,10.09.1985, H.J. Chawdhary 76267 (BSD); Sobla, 08.08.2004, S.K. Srivastava 105907 (BSD); Bungling, 04.08.1998, B.P. Uniyal \& Bipin Balodi 93811 (BSD, DD); Lilam, 15.09.1983, Bipin Balodi 75241 (BSD); Maitli, 01.10.2001, M.S. Pundir 97034 (BSD); Tawaghat, 09.1986, Bipin Balidi 83210 (BSD); Uttarkashi district, Govind Pashu Vihar National Park \& Sanctuary, Thalluka, 24.09.2010, R. Manikandan 113706 (BSD); Mori, 17.09.1995, Surendra Singh 89861 (BSD); on way to Sangam, 07.1999, Bipin Balodi 95004 (BSD); on the way to Thalluka, 19.09.1995, Bipin Balodi 89349 (BSD). West Bengal, Darjeeling district, Kalimpong, 14.09.1914, Thornton Ripley s.n. (CAL); Ibid., 17.09.1914, Thornton Ripley s.n. (CAL); Rimbik, 06.08.1981, B. Krishna 1629 (CAL); Rishyap, 23.10.1870, C.B. Clarke s.n. (CAL); Sureil, 21.09.1908, W.W. Smith 006 (CAL).

Notes: O. stellata can be easily recognized by the more or less ' $S$ ' shaped large anthers with long beak, urceolate hypanthium with dense emergences and hairs. However, wide variations in vestiture created confusion in the delimitation of taxa and often resulted in the description of too many taxa which are not tenable. Presently two varieties have been recognized under O. stellata such as var. crinita and var. hispidissima. Var. crinita differs from the latter by patent hairy stems and green emergences on the hypanthium. Similarly, var. hispidissima differs from var. stellata and var. crinita by its reddish brown emergences onthe hypanthium.

Osbeckia stellata Buch.-Ham. ex D.Don var. crinita (Benth. ex Naudin) C.Hansen, Ginkgoana 4: 31. 1977. O. crinita Benth. ex Naudin, Ann. Sci. Nat. Bot., ser. 3, 14: 72. 1850; C.B.Clarke in Hook.f., Fl. Brit. India 2: 517. 1879; Kanjilal et al., Fl. Assam. 2: 292. 1983; N.P.Balakr., Fl. Jowai \& Vicinity 206. 141. 1983. Lectotype. (designated by Hansen, 1977): INDIA, Assam, s.d., M. Walker s.n. (P02441145 image!).

O. cinerea Cogn. in A.DC. \& C.DC., Monogr. Phan. 7: 1177. 1891. Type: BANGLADESH, Sylhet, 1887, M. Balansa 4055 (holo P02441151 digital image!).

O. paludosa Craib, Bull. Misc. Inform., Kew. 262. 1916. Type: THAILAND, Siam, Chiengmai, 330 m, 21.10.12, A.F.G. Kerr 2740 (holo K000867937 digital image!).

O. garrettii Craib, Bull. Misc. Inform. Kew. 262. 1916. Type: THAILAND, Doi Kar Intanon, $18^{\circ}$ 40’ N, $98^{\circ} 30^{\prime}$ 'S, 1050 m, 16.09.1910, H.B.G. Garrett 51 (holo K000867935 digital image!).

O. robusta Craib, Notes Roy. Bot. Gard. Edinburgh 10: 54. 1917. Type: CHINA, Yunnan, s.d., Forrest 8561 (holo E00225554 digital image!).

O. mairei Craib, Notes Roy. Bot. Gard. Edinburgh 10: 54. 1917. Type: CHINA, Yunnan, 690 m, s.d., E.E. Maire s.n. (holo E00225556 digital image!).

O. septemnervia Buch.-Ham. ex Craib, Notes Roy. Bot. Gard. Edinburgh 10: 55. 1917. Type: INDIA, Assam, Goalpara, s.d., Hamilton 916 (holo E00225749 digital image!). 
O. yunnanensis Franch. ex Craib, Notes Roy. Bot. Gard. Edinburgh 10: 57. 1917. Type: CHINA, Yunnan, 2500 m, 26.09.1884, Delavay 191 (holo P02441147 digital image!).

O. sikkimensis Craib, Notes Roy. Bot. Gard. Edinburgh 10: 56. 1917. Type: INDIA, Sikkim, Yoksom, 10.10.1875, C.B. Clarke 25207B (holo E00225751 digital image!).

O. wattii Craib, Notes Roy. Bot. Gard. Edinburgh 10: 57. 1917. Type: INDIA, Nagaland, Naga hills, 05.1882, Watt 7280 (holo K000890023 digital image!).

O. pulchra E.T.Geddes, Bull. Misc. Inform., Kew 1930(4): 171. 1930. Type: THAILAND, (Doi Sootep), 1600 m, 24.11.1912, Kerr s.n., (holo ABD!).

O. pulchra var. rubra Craib, Fl. Siam. Enum. 1: 677. 1931. Type: THAILAND, Siam, Doi Sootep, 1200 m, 17.11.1922, Kerr s.n. (holo BM000944334 digital image!).

Melastoma crinitum Roxb., Fl. Ind., ed. 2, 2: 402. 1832, non Vahl, 1807. Type: INDIA, s.loc., 05.1811, Roxburgh 347 (holo BM000944403 digital image!).

M. crinitum Vahl, Eclog. Amer. 3: 28. 1807. Type: Not seen.

M. crinitum Naudin, Ann. Sci. Nat., Bot. ser. 3, 13: 280. 1850. Type: Not seen.

M. mairei H.Lév., Repert. Spec. Nov. Regni. Veg. 11: 300. 1912.

O. crinita Benth. in Wall., Numer. List 143, no. 4066. 1831, nom. nud.

O. septemnervia Buch.-Ham. in Wall., Numer. List. 4062 B. 1831, nom. nud.

Fig. 22

Erect, perennial shrubs, 0.50-2 m tall. Stems branched, young branches quadrangular, with patent, slightly reflexed brown hairs. Petioles 4-8 mm long; lamina ovate to broadly ovate, 4-13 $\times$ 2-4 cm, 5-nerved, green, apex acute to attenuate, base acute to rounded, both sides densely clothed with patent hairs. Cymes terminal, 6-12-flowered; bracts ovate to broadly ovate, 4-8 $\mathrm{mm}$ long, ciliate, with few to many appressed hairs dorsally. Flowers tetramerous; pedicels $0.5-3 \mathrm{~mm}$ long. Hypanthium urceolate, 7-12 × 2.5-6 mm, covered with emergences, bristles and patent or appressed hairs; intersepalar emergences 4-7 mm long, with a terete stalk bearing a few to many patent hairs and terminating in a stellate head, caducous; other emergences differ from intersepalar emergences, smaller. Sepals 4, narrowly triangular, 7-13 × 1.5$3 \mathrm{~mm}$ long, ciliate with a few to many patent hairs dorsally, caducous. Petals 4, obovate to broadly obovate, 13-18 × 10-15 mm long, glabrous, margins ciliate, pale pink to purple. Stamens 8; filaments 7-14 mm long; anthers 'S' shaped, 11$15 \mathrm{~mm}$ long, yellow, narrowly ovate, with long beak; pore oblique on ventral side; connective prolonged into a slightly undulating collar with large dorsal lobe. Ovary united with hypanthium for about two thirds of its length, anther pockets extending to the base of the ovary; free part sometimes glabrous, but more often thinly clothed with short appressed hairs; style 15-25 mm long, glabrous. Capsules urceolate, 7-11 × 2-6 mm, neck often widening towards rim, densely hairy below, more or less glabrous above; hypanthium exceeds ovary and crown; seeds numerous, 0.1-0.2 mm long, minute, curved.

Flowering \& fruiting: July-January.

Chromosome number: $n=10$ (Meenakumari \& Kuriachan, 1990)

Habitat: In evergreen forests at an elevation above $500 \mathrm{~m}$ is association with O. stellata var. stellata and O. capitata.

Distribution: Bhutan,India (Arunachal Pradesh, Assam, Manipur, Meghalaya, Nagaland, Sikkim, West Bengal) and Nepal.

Specimens examined: INDIA, Arunachal Pradesh, Lohith district, Chowkham, 24.11.1969, J. Joseph 48314 (ASSAM); Nansai township, 09.09.1969, A.S. Rao 47716 (CAL, ASSAM); Paya nava, 17.11.1957, R.S. Rao 10674 (ASSAM); Lower Subansiri district, Khorbar, 28.09.1959, G. Panigrahi 19732 (ASSAM); Subansiri, 14.11.1946, A.R.K. Sastry 40609 (ASSAM); Tirap district, Deomali, West of Khonsa, 


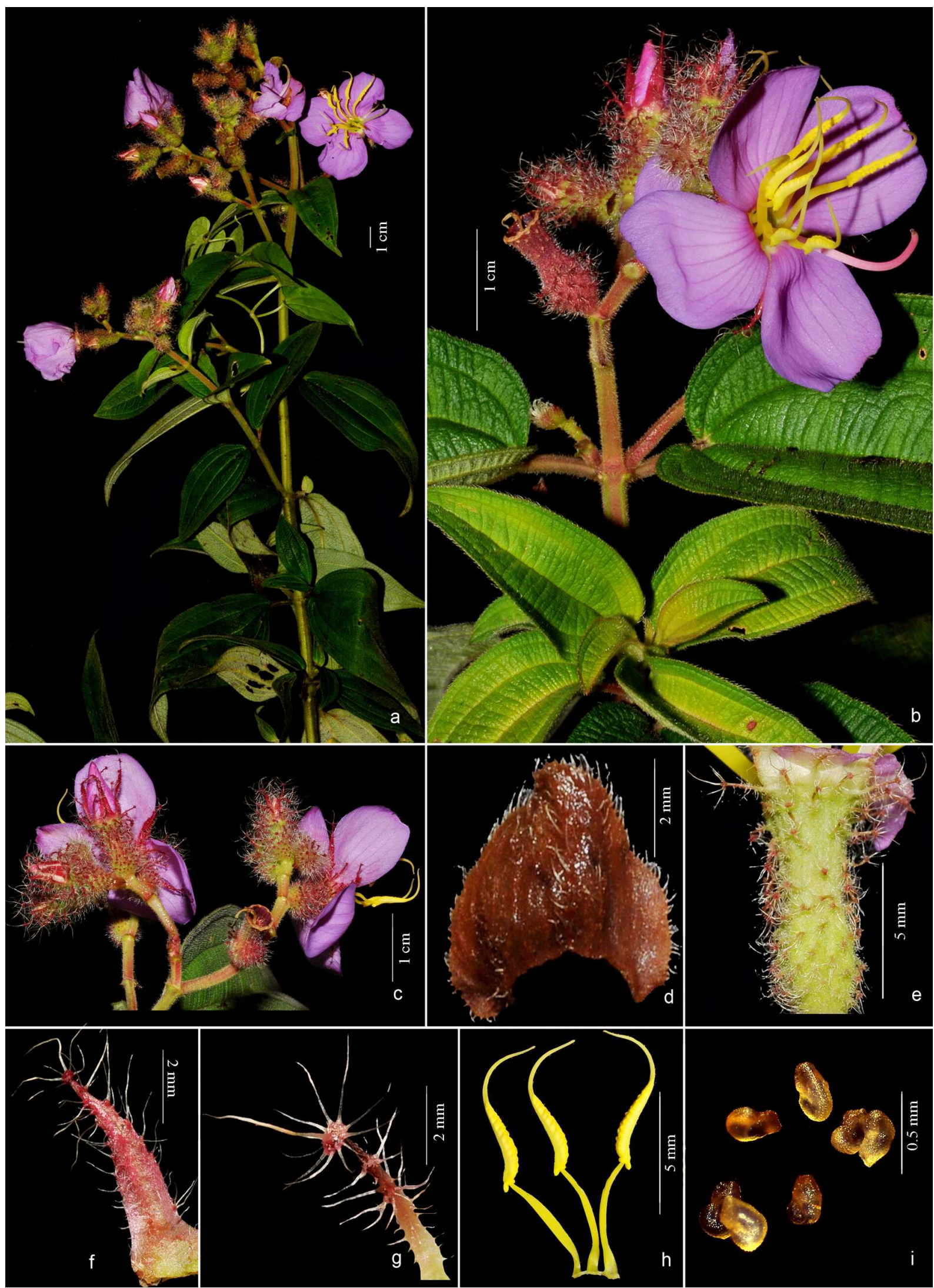

Fig. 22. Osbeckia stellata Buch.-Ham. ex D.Don var. crinita_(Benth. ex Naudin) C. Hansen: a \& b Habit; c. Inflorescence; d. Bract; e. Hypanthium; f. Calyx lobe; g. Intersepalar emergence; h. Stamens; i. Seeds. 
21.06.1961, F.B. Dub 25886 (ASSAM); West Siang district, Dirang, 17.09.1964, J. Joseph 40058 (ASSAM); Kiak, 25.11.1958, R.S. Rao 18022 (ASSAM); Klaktang, 08.08.1973, P.K. Hajra 54019 (ASSAM); Ibid., 14.10.1973, P.K. Hajra 54113 (ASSAM); Lumla, 24.08.1976, P.K. Hajra 68481 (ASSAM); Sepa, 29.10.1970, A.S. Rao 49425 (ASSAM). Assam, Dima Hasao district, Garodong Bhutan border, 18.09.2010, C. Deori \& D.K. Ray 119022 (ASSAM); Doboka, 28.06.1963, D.B. Deb 35403, 35404 (ASSAM); East Karbi Anglong district, Phulai, 26.06.1963, D.B. Deb 35378 (ASSAM); Ibid., 17.09.2010, C. Deori \& D.K. Ray 119122 (ASSAM); Golaghat district, Kaziranga N.P., 14.08.1975, P.K. Hajra 52887 (ASSAM); Jorhat district, Borbheta, Phutuka, 11.03.1959, S.N. Bora 33643 (ASSAM); Khatangpani R.F, 09.07.1959, G. Panigrahi 18905 (CAL); Makum, 21.11.1911, I.H. Burkill 35746 (CAL); Orang Barseola Bank of Bihari River, 22.07.1958, G. Panigrahi, 14220 (CAL, ASSAM); Kamrup district, 24.10.1935, G.K. Deka 12848 (ASSAM); Lakhimpur district, near NEFA I.B., 12.05.1966, D.M. Verma 41724 (ASSAM); Nagaon district, Sonaibuchi R.F., 20.08.1964, N.P. Balakrishnan 39300 (ASSAM). Manipur, Imphal district, Chingmairong, 01.08.1952, D.B. Deb 777 (CAL); Ibid., 27.10.1952, D.B. Deb 546 (CAL); Pipihma, 02.1906, Meebold 5605 (CAL); Myanglihing, 20.05.1882, George Watt 7157 (CAL); Senapati district, Chingmei Hills, 22.12.1976, Barin Gherh 11602 (CAL). Meghalaya, Changlang, Khela, 19.08.1958, G. Panigrahi 14415 (CAL, ASSAM); Cherrapunji, 18.10.1932, H.K. Nandi s.n. (CAL); Ibid., 27.09.1955, G. Panigrahi 3488 (CAL, ASSAM); Ibid., 31.10.1972, R.S. Rao 47279 (CAL); East Khasi hills district, Barapani, 11.09.1970, B.K. Sinha 112013 (ASSAM); Jowai district, Jowai, 26.05.1956, R.S. Rao 2571 (CAL, ASSAM); Khasi hills, Old Barapani Road, 11.09.1970, A.S. Rao 38637 (CAL); Jaintia Hills district, Raliang to Garampani, 22.08.1968, N.P. Balakrishnan 46986 (ASSAM); Khasia \& Jaintia Hills, Shillong, 26.10.1915, Upendranath Kanjilal 6134 (ASSAM); Ibid., 26.05.1956, R.S. Rao 2571 (CAL); Laithkor, 28.06.2014, Prashob \& Sibichen
6113 (DEV); Ibid., 11.09.2015, Prashob \& Sibichen 6123 (DEV); Mahadavkala, 21.11.1962, S. Choudary 25157 (CAL); Mawphlang, Law-Hyngowh, 20.11.1972, P.K. Hajra 51922 (CAL, ASSAM); Mawphlang Sacred forest, 09.09.1972, P.K. Hajra 51948 (CAL, ASSAM); Ibid., 17.09.2015, Prashob E Sibichen 6139 (DEV); Mawsynram, 25.02.1960, G. Panigrahi 21264 (ASSAM); Pynursula, 04.12.1943, N.L. Bor 18079 (DD); Sohra, 27.06.2014, Prashob \& Sibichen 6106 (DEV); Ibid., 20.09.1939, S.R. Sharma 9411 (ASSAM); Shillong, 25.05.1911, I.H. Burkill \& S.C. Banerjee s.n. (CAL); Ibid., 26.05.1911, I.H. Burkill \& S.C. Banerjee 28 (CAL); Ibid., 22.06.1913, Upendranath Kanjilal 2218 (ASSAM); Ibid., 12.09.1913, Upendranath Kanjilal 2628 (ASSAM); Ibid., 23.09.1913, Upendranath Kanjilal 2618 (ASSAM); Ibid., 29.09.1915, H.G. Carter 41409 (CAL); Ibid., 12.10.1931, S.R. Sharma 9547 (ASSAM); Ibid., 25.10.1938, K. Biswas 3730 (CAL); Ibid., 02.10.1945, N.L. Bor 18436 (DD); Ibid., 02.10.1945, N.L. Bor 18445 (DD); Ibid., 25.11.1949, B.B. Syam 22908 (ASSAM); Ibid., 26.11.1952, S. Choudary 25263 (CAL, ASSAM); Ibid., 30.08.1956, G. Panigrahi 3199 (CAL, ASSAM); Ibid., 20.06.1958, G. Panigrahi 16497 (ASSAM); Ibid., 02.09.1960, G.K. Deka 21761 (ASSAM); Ibid., 13.09.1960, G.K. Deka 21762 (ASSAM); Ibid., 09.08.1962, G.K. Deka 17090 (ASSAM); Ibid., 25.09.1962, S.L. Kapoor 73449 (CAL); Ibid., Ibid., 26.11.1962, S. Choudhary, 25227, (CAL, ASSAM); 10.12.1962, R.M. Dutta 25436 (ASSAM); Ibid., 12.12.1962, S.K. Kotari 27944 (ASSAM); Ibid., Behind Bishop falls, 24.12.1962, R.M. Datta 25533 (ASSAM); Ibid., 24.12.1962, S. Choudary 25234 (CAL); Ibid., 24.12.1962, R.D. Ulla 25534 (CAL); Ibid., 28.12.1962, S.K. Kotari 29613 (ASSAM); Ibid., s.n., R.S. Rao 1693 (CAL, ASSAM); Shillong, Mulki village, 19.11.1962, S.K. Kar 29486 (ASSAM); Shillong peak forest, 26.10.1960, D.C.S. Raju 22015 (ASSAM); Ukhral, 28.07.1948, S.K. Mukerjee 3245 (CAL); Ibid., 22.09.1948, S.K. Mukerjee 3528 (CAL); Upper Shillong, 29.09.1915, H.G. Carter 41409 (CAL); Woodland, Shillong, 26.11.1962, s.coll. 25234 (CAL, ASSAM); Ibid., 10.09.1965, R.V. Kammathy 001 (CAL); Ummulong, 16.08.1968, N.P. Balakrishnan 42990 
(ASSAM); Ri-Bhoi district, Nongpoh 23.09.1935, S.R. Sharma 12397 (ASSAM); Ibid., 23.07.1938, S.R. Sharma 17951(ASSAM); Ibid., 24.08.1938, S.R. Sharma 18167(ASSAM); G. Panigrahi 4348 (ASSAM); Ibid., 30.07.1964, J. Joseph 37475 (ASSAM); West Khasi Hills district, Nongstom, 01.04.1962, G.V. Subba Rao 28470 (ASSAM). Mizoram, Aizawl district, Buia Hmun Tlang, 11.11.1999, A.A. Mao 107153 (ASSAM); Dampa Tiger reserve, Teirei, 21.09.2006, B.K. Sinha \& N. Odyuo 112738 (ASSAM); Dampa Tiger reserve, Way to Chhautpier top, 28.05.2005, N. Odyuo 109747 (ASSAM); Lawngtlai district, Kawlkhul, 04.10.1989, K.P. Singh 94334 (ASSAM); Kolasib, Bhairawi road, 31.05.1988, K.P. Singh 93191 (ASSAM); Ibid., 16.10.1989, K.P. Singh 92861 (ASSAM); Mizo hills, 12.07.1963, Thalthlone, R.M. Dutta 33925 (ASSAM); Ibid., Vawmbuk, 12.07.1963, R. Dutta 33929 (ASSAM); Zote, 10.10.1989, K.P. Singh 94574 (ASSAM); s.loc, 29.04.1998, S.L. Abbar 102615 (ASSAM). Nagaland, Kohima district, Jasma, 12.1955, B.F.O. s.n. (ASSAM); Lower Japfu hill, 05.09.2003, A.A. Mao \& R. Gogoi 112504 (ASSAM); Naga hills, 06.1935, N.L. Bor 21002, 21000, 21009 (ASSAM); Ibid., 12.1935, N.L. Bor 6213 (DD); Ibid., 06.1936, N.L. Bor 10557 (ASSAM); Ibid., Lzulake valley, 09.1939, N.L. Bor 90574 (ASSAM); Ibid., 25.11.1942, N.L. Bor 17284 (ASSAM); Ibid., 30.11.1986, R.C. Gauri 773 (DD). Sikkim, East Sikkim district, North East of Gangtok, 08.11.1960, K. Jhothathri 9475 (CAL); Gangtok, 26.08.1980, P.K. Hajra 865 (BSHC); Takchi, 18.07.1985, D.C.S. Raju 7727 (BSHC); Takchi, Luing, 16.07.1998, S.K. Rai 23404 (BSHC); Tashi view point, Gangtok, 26.08.1980, P.K. Hajra 866 (CAL); North Sikkim district, s.loc., 09.1879, J.S. Gamble s.n. (DD); North Sikkim, 11.1879, J.S. Gamble s.n. (DD); South Sikkim district, s.loc., 18.09, S. Kurz 171902 (CAL). Rabongla, 23.08.1986, A.K. Verma 12701 (BSHC); South Sikkim, s.d., S. Kurz s.n. (DD); West Sikkim district, DamthangRawangla, 08.1991, R. Gopalan 97559 (BSHC); Karchi researve forest, 15.12.1994, G.P. Sinha \& S. Pradhan 10862 (BSHC); Ribdi, 07.09.1995, R. Nath
\& S.K. Jana 31548 (BSHC); Rengu temple, J.D. Hooker 1867 (CAL); Sikkim, s.loc., 14.08.1870, C.B. Clarke s.n. (CAL); Ibid., s.d., R.S. Rao 319 (CAL). West Bengal, Darjeeling district, Birchi hills, Darjeeling, 12.11.1944, M.B. Raizada 18939(DD); Ibid., 22.11.1962, J.K. Maheshwari 5018 (CAL); Chungtang, 26.04.1965, H. Santapau \& S.K. Mukerjee 107 (CAL); Darjeeling, 09.1879, J.S. Gamble 7068 (DD);Ibid., 09.1880, J.S. Gamble 8361 (DD); Ibid., 01.10.1902, J.H. Lacek 2382 (CAL); Ibid., 01.10.1902, J.H. Lace 2382 (DD); Ibid., 22.09.1962, B. Sahni 1802 (CAL); Ghoong, 07.1996, Sanjoy Ghara 006 (CAL); Jorbangla, 16.04.1961, s.coll. 111 (CAL); Singalila National park, Meghma, 15.09.2002, A.K. Ghosh 30621 (CAL).

Notes: Osbeckia stellata var. crinita differs from var. stellata and var. hispidissima by its patent hairy stem, shape, colour and size of the hypanthium. The first available name for the taxon was Melastoma crinitum Roxb. (1832). However, it is illegitimate being a later homonym of M. crinitum Vahl. (1807). Prior to the publication of Roxburgh, Wallich (1831) included O. crinita Benth. in his catalogue. However, Wallich's name is not valid as it is not accompanied by a description, diagnosis, or a reference to a published diagnosis. Later Naudin (1850) described the taxon as O. crinita Naudin, instead of O. crinita Benth. ex Naudin.,without mentioning Bentham. Being a variety of O. stellata Buch.-Ham. ex D.Don. and the first legitimate specific epithet in the genus Osbeckia, i.e. crinita Benth. ex Naudin, the correct name becomes $O$. stellata var. crinita (Benth. ex Naudin) C. Hansen.

Osbeckia stellata Buch.-Ham. ex D.Don var. hispidissima (Wight) C.Hansen, Ginkgoana 4:37. 1977. O. hispidissima Wight, Icon. Pl. Ind. Orient. t. 1612. 1850; Gamble, Fl. Pres. Madras 1: 493. 1919; Pull., D.M.Rao \& K.S.Ramamurthy, Fl. Eastern Ghats 57. 2002; G.V.S.Rao \& G.R.Kumari, Fl. Visakhapatnam Dist. Andhra Pradesh 340. 2003. Type: INDIA, s. loc., 25.11.45, Cleghorn D-46 (holo K000890022 image!).

Fig. 23

Erect, perennial shrubs, 0.5-3.5 m tall. Stems branched, quadrangular; hairs long and stiff, patent 


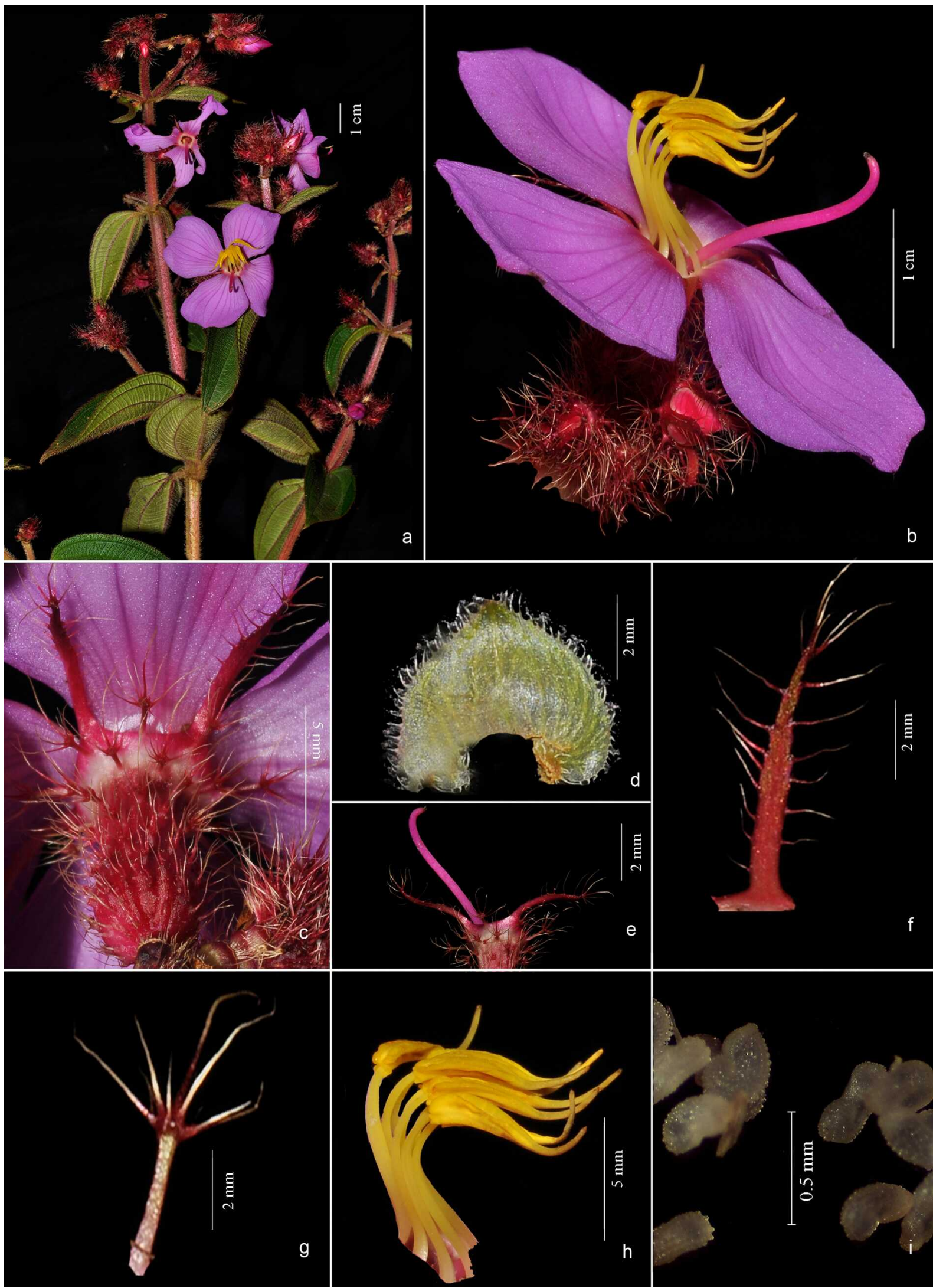

Fig. 23. Osbeckia stellata Buch.-Ham. ex D.Don var. hispidissima (Wight) C. Hansen: a. Habit; b. Flower; c. Hypanthium; d. Bract; e. Style; f. Calyx lobe; g. Intersepalar emergence; h. Stamens; i. Seeds. 
at the base, curved upwards. Petioles $5-11 \mathrm{~mm}$ long; lamina narrowly to broadly ovate, $8.2 \times 3.1$ $\mathrm{cm}, 5$-nerved, apex acute to attenuate, base acute, indumentum thin to dense, hairs ascending to patent on both sides. Cymes, 5-10-flowered; bracts green, small, $4 \mathrm{~mm}$ long, hairy. Flowers tetramerous; pedicels $0.5-3 \mathrm{~mm}$ long. Hypanthium urceolate, $9-11 \times 2.5-3 \mathrm{~mm}$, covered with patent emergences and a few bristles at the base; intersepalar emergences 5-7 mm long, with a terete glabrous stalk, terminating in a stellate head; other emergences similar but smaller, restricted to uppermost part of hypanthium mixed with appressed hairs. Sepals 4, lanceolate to ovate, margins ciliate or hairy, both sides glabrous, apex stellate. Petals 4, obovate to broadly obovate, 16-28 mm long, pink to purple, glabrous, apex ciliate. Stamens 8; filaments 8-12 mm long, glabrous; anthers narrowly ovate, 'S'shaped, yellow, 10-15 mm long, beak narrow, 2$3 \mathrm{~mm}$ long, not twisted; pore oblique; connective with undulating collar having large dorsal lobes. Ovary united with hypanthium for about two thirds of its length, anther pockets extending to the base of the ovary, crown appressed hairy; style 13-18 mm long, glabrous. Capsules urceolate, 8$10 \times 2.5-3 \mathrm{~mm}$, neck widens towards rim, densely covered with emergences and hairs which often fall off when mature; seeds 0.1-0.2 mm long, numerous, curved, muricate.

\section{Chromosome number: Not reported}

Habitat: Evergreen forests at an elevation above $500 \mathrm{~m}$. in association with O. stellata var. stellata.

Flowering \& fruiting: August-February.

Distribution: Endemic to India.

Specimens examined: INDIA, Karnataka, Chikmagalur district, Bababoodan Hills, 25.11.1845, Meebold 10464 (CAL); Aglatti, Mysore, 11.1908, s.coll. s.n. (JCB); Hassan district, Bisle Ghat, 25.4.1970, Saldanha 16879 (JCB); Vanagw, 15.05.1968, Saldanha 11718 (JCB). Meghalaya, East Khasia Hills district, Air force campus, Shillong, 27.10.15, Prashob E Sibichen 6623 (DEV); Shillong, 27.10.15, Prashob E Sibichen 6622 (DEV).
Notes: This variety is closely related to O. rostrata and O. stellata var. crinita, resembling the former by the hypanthium and the latter by the patent hairy stem. The reddish brown colour of the hypanthium, intersepalar emergences with glabrous stalk and inflexed bristles on the stem justifies its varietal status.

\section{Osbeckia sect. Osbeckia}

The section comprises annual herbs having tetra merous flowers and long beaked anthers. Triana (1871) included 4 species viz., Osbeckia chinensis L., O. capitata D.Don ex Wight \& Arn., O. zeylanica L.f. and O. perottetii D.C. ex Naudin under the section Genuinae. Clarke (1879) also included the first 3 species under the same section. Cogniaux (1891) followed Triana and included 4 species in the section Genuinae in "Monographie Phanerogamarum". However the sectional name Genuinae is inadmissible as it is contrary to Art. No. 22.1, 22.2 of ICN (Turland et al., 2018), hence the sectional name "Osbeckia", is used in the present treatment instead of "Genuinae". This section is represented by three species and one variety in India.

\section{Osbeckia sect. Osbeckia}

Genuinae Triana, Trans. Linn. Soc., London 28: 53. 1871; C.B.Clarke in. Hook.f., Fl. Brit. India 2(6): 515. 1879; Cogn. in A.DC. \& C.DC., Monogr. Phan. 7: 325. 1891. Type: Osbeckia chinensis L.

Annual herbs; Flowers tetramerous; anthers long beaked.

Distribution: Distributed mainly along wet tropical and subtropical Asia including Indo China, Nepal, Bhutan, and Sri Lanka.

\section{Key to the species of sect. Osbeckia}

1a. Hypanthium campanulate with emergences and hairs 2

1b. Hypanthium urceolate, glabrous or occasionally hairy.... O. capitata 
2a. Intersepalar emergences persistent, prominently stalked, margins not ciliate O. zeylanica

2b. Intersepalar emergences caducous, not stalked, margins ciliate O. chinensis

Osbeckia capitata D.Don ex Wight \& Arn., Prodr. Fl. Ind. Orient. 1: 323. 1834; Walp., Nov. Actorum Acad. Caesp. Leop.-Carol. Nat. Cur. 19. Suppl. 1: 331. 1843; Naudin, Ann. Sc. Nat. Ser. 3. 14. 1850; C.B.Clarke in Hook.f., Fl. Brit. India 2: 517.1879; C.Hansen, Ginkgoana 4: 47. 1977; N.P. Balakr., Fl. Jowai \& Vicinity 206. 141. 1983. Lectotype (designated by Hansen, 1977): BANGLADESH, Sillhet, s.d., Wallich 4072 (K000890015, digital image!)

O. glauca Benth. ex Naudin, Ann. Sci. Nat. Bot. Ser. 3. 14: 68. 1850, non Benth. ex Triana, 1871. Type: BANGLADESH, Sillhet, s.d., Wallich s.n. (holo P02441104, digital image!).

Fig. 24

Annual herbs, main stem prostrate with erect branches, 7-50 cm tall. Stems quadrangular, branched or unbranched, covered with appressed, hairs. Leaves sub-sessile; petioles 0.2-1.2 mm long; lamina ovate to broadly ovate, $1-3 \times 0.5-2.3 \mathrm{~cm}$, apex acute, base obtuse to subcordate, 3-5-nerved, both sides clothed with ascending short hairs. Cymes terminal, cluster of 2-3 flowers or solitary, subtended by 1 or 2 pairs of leaves. Flowers tetramerous; pedicels to $1 \mathrm{~mm}$ long. Bracts ovate to elliptic, 3.5-6 $\mathrm{mm}$ long, margins ciliate, appressed-hairy dorsally. Hypanthium urceolate, 6$8 \times 3-5 \mathrm{~mm}$, glabrous or occasionally hairy, with emergences; intersepalar emergences scale-like, 1$2.5 \times 0.5-1 \mathrm{~mm}$, caducous, rounded at apex, without a distinct head, length of hairs on margins increases towards the tip, dorsal side clothed with appressed hairs. Sepals 4, triangular or ovate, 6-10 $\mathrm{mm}$ long, caducous, dark brown to green in color with indistinct nerves, margins ciliate, dorsal side clothed with appressed-hairs. Petals 4, pink, obovate, 10-17 × 6-11 mm, 10-12-nerved, apical margins ciliate. Stamens 8; filaments $5-11 \mathrm{~mm}$ long, dark pink; anthers ovate, 5-8 mm long, yellow, narrowed into a slender, tubular beak 2-4.5 mm long, not twisted, pore oblique on the ventral side of tip, narrow, connective prolonged into a collar. Ovary united with hypanthium for about half of its length; anther pocket extending to the base of ovary; free part glabrous, crown distinct; style 14-17 mm long. Capsules urceolate, 7-9 $\times 3.5-5 \mathrm{~mm}$, not ribbed; seeds $0.2-0.4 \mathrm{~mm}$ long, numerous, curved.

Flowering \& fruiting: August-October.

Chromosome number: $n=10$ (Meenakumari \& Kuriachan, 1990).

Habitat: This species thrives best in elevation varying from 500-1500 m., commonly seen in boarders of evergreen forests as well as grasslands, occurs in massive number in association with $O$. stellata var. stellata and O. stellata var. crinita.

Distribution: China, Bhutan and India.

Specimens examined: INDIA, Assam, Dima Hasao district, Umrangso, N.C. Hills, 12.01.2007, R.S. Baruah 115222 (ASSAM). Meghalaya, Cherrapunji, 27.09.1956, G. Panigrahi 3480 (CAL); Cherrapunji, way to Nokthlang, 30.07.1913, Upendranath Kanjilal 2365 (ASSAM); East Khasi Hills district, Mawphlanag, Hill slope, 29.10.1972, P.K. Hajra 51907 (CAL); Jaintia hills district, Dawki, 12.09.2015, Prashob \& Sibichen 6134 (DEV); Jarain pitcher plant lake, 19.09.2015, Prashob \& Sibichen 6143 (DEV); Jowai, 03.06.1952, A. Das 39288 (ASSAM); Ibid., 19.09.2015, Prashob \& Sibichen 6149 (DEV); Khasia \& Jaintia Hills, 13.07.1913, Ranjilal 2365 (ASSAM); Ibid., 22.08.1935, G.K. Deka 13082 (ASSAM); Laitkor Shillong, 28.06.1963, S.K. Kar 18072 (ASSAM); 28.06.2015, Prashob \& Sibichen 6112 (DEV); Ibid., 26.09.2016, Prashob \& Sibichen 6627 (DEV); Mawphlang sacred forest, 17.09.2015, Prashob \& Sibichen 6140 (DEV); Mawsynram, 16.09.2015, Prashob \& Sibichen 6138 (DEV); Nongtalang village, 19.09.2015, Prashob \& Sibichen 6146 (DEV); Nongthymai, 20.09.1956, G. Panigrahi 3297 (ASSAM); Pynursula, 25.08.1956, G. Panigrahi 2982 (ASSAM); Pynursula, way to Sohra, 27.06.2015, Prashob E Sibichen 6110 (DEV); 


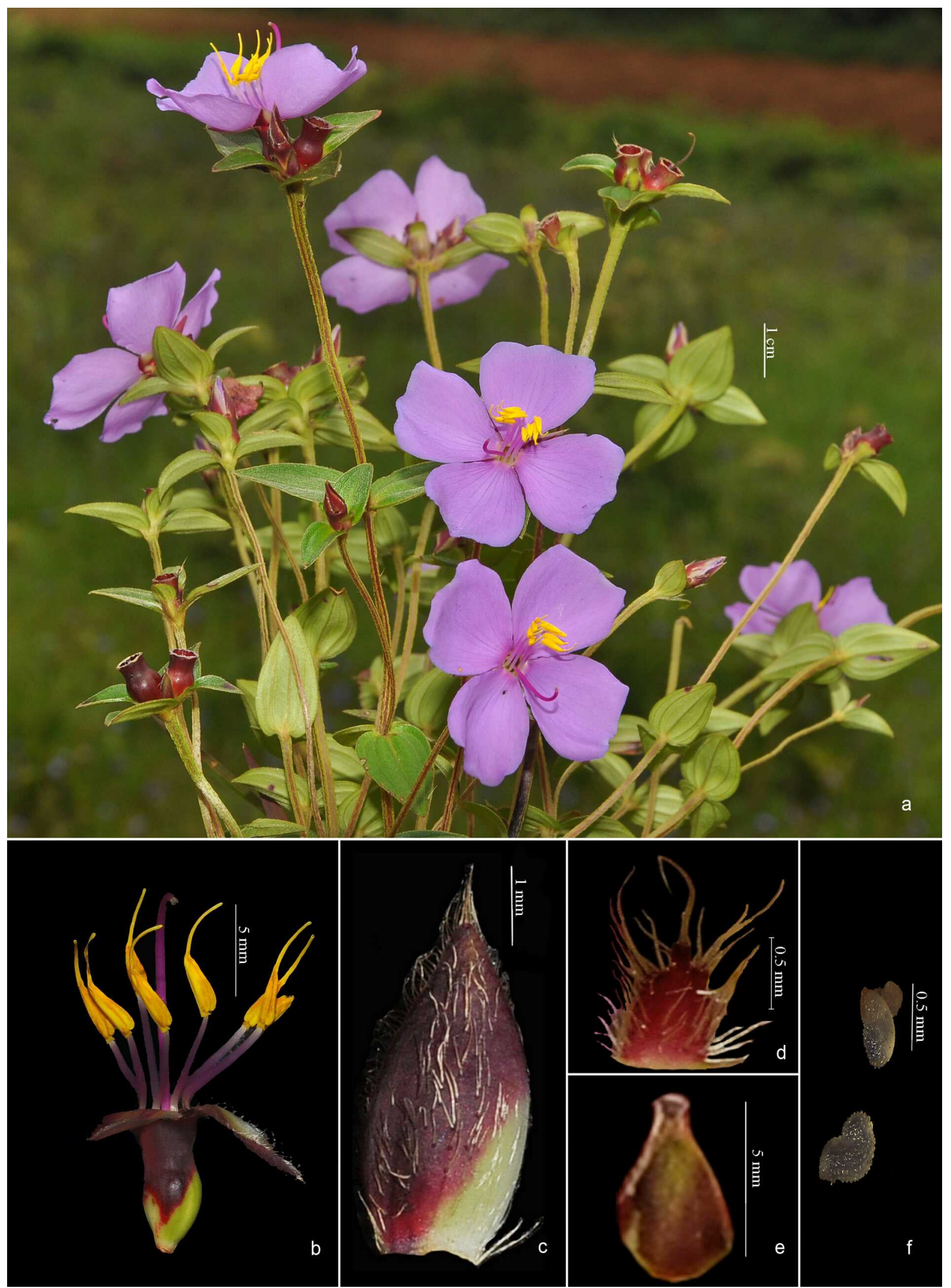

Fig. 24. Osbeckia capitata D.Don ex Wight \& Arn.: a. Habit; b. Hypanthium with stamens; c. Calyx lobe; d. Intersepalar emergence; e. Bract; f. Seeds. 
Shillong, 20.09.1956, G. Panigrahi 3297 (CAL); Ibid., 09.07.1943, N.L. Bor 17965 (DD); Ibid., 24.07.1971, Ramesh Bedi 434 (DD); Ibid., 24.09.1885, C.B. Clarke 40517 (CAL); Ibid., 26.05.1911, I.H. Burkill \& S.C. Banerjee 42 (CAL); Ibid., 29.05.1911, I.H. Burkill \& S.C. Banerjee 82 (CAL); Ibid., 29.09.1915, H.G. Carter 41408 (CAL); Shillong, Laitkor, 28.06.1963, S.K. Kar 18073 (CAL); Shillong, Woodland, 16.07.1964, H. Deka 36912 (ASSAM); Shillong peak, 26.10.1960, D.C.S. Raju 22023 (ASSAM); Sohra, 11.09.1940, R.N. Desai 2044 (ASSAM); Ibid., 26.09.2016, Prashob E Sibichen 6628 (DEV); Ibid., 06.09.1962, G.K. Deka 23376 (ASSAM); Ummulong to Jowai, 16.08.1968, N.P. Balakrishnan 46105 (ASSAM); Upper Shillong, 24.08.1958, R.S. Rao 14034 (CAL, ASSAM); West Khasi hills district, Markasa village, 18.06.1958, G. Panigrahi 16389 (CAL, ASSAM). Mizoram, Lunglei district, Mizo hills, Darzo to Leite, 18.07.1963, R. Dutta 34102 (ASSAM).

Notes: Osbeckia capitata resembles $O$. chinensis in having tetramerous, pink flowers with caducous sepals but the latter can be readily distinguished by its sub-sessile ovate leaves, as opposed to the petiolate, linear-lanceolate leaves of the former.

Osbeckia chinensis L., Sp. P1. 345. 1753; C.B.Clarke in Hook.f., Fl. Brit. India 2(6): 515. 1879; C.Hansen, Ginkgoana 4: 49. 1977; T.Pullaiaih \& Ramam., Fl. East. Ghats, India. 56. 2001; N.P.Balakr. Fl. Jowai \& Vicinity 206. 141. 1983; R.S.Rao, Fl. East Godavari Dist., 366. 1999; T.Pullaiah, D.M.Rao \& K.S.Ramam., Fl. East. Ghats 56. 2002; G.V.S.Rao \& G.R.Kumari, Fl. Visakhapatnam Dist., Andhra Pradesh, 339. 2003. Lectotype (designated by Hansen, 1977): s.loc, s.d., P. Osbeck s.n. (LINN digital image!).

O. sessilifolia Stokes, Bot. Mat. Med. 2: 348. 1812. Type: Not seen.

O. chinensis L. var. gerzuina Kurz., J. Asiat. Soc. Bengal. 2. Nat. Hist. 46: 74. 1877. nom.illeg. Type: Not seen.

O. chinensis L. var. typica Hochr., Candollea 2: 469. 1925. nom. illeg. Type: Not seen.
O. angustifolia D. Don, Prodr. Fl. Nepal. 221.1825. Osbeckia chinensis var. angustifolia (D.Don) C.Y.Wu \& C.Chen, Fl. Yunnan. 2: 80. 1979. Type: Not seen.

Tristemma angustifolium Blume, Bijdr. Fl. Ned. Ind. 17: 1079. 1826. Type: Not seen.

O. linearis Blume, Flora 14: 473. 1831. Melastoma linearis Reinw. ex Blume, Flora 14: 474. 1831. (prosyn.). Osbeckiachinensis var. linearis (Blume) Kurz, J. Asiat. Soc. Bengal pt. 2. Nat. Hist. 48: 74. 1877. Osbeckia linearis Blume var. moluccana Blume, Mus. Bot. Lugduno-Batavum 1: 51. 1849. Lectotype (designated here): Indonesia, s.d., s.coll. s. n. (K000867922 digital image!).

O. ciliaris Ser. ex DC., Prodr. 3: 132. 1828. Type: Not seen.

O. multiflora Blanco, Fl. Filipinas 293. 1837. Type: Not seen.

O. myrtifolia Blume, Mus. Bot. 1(4): 51. 1849. Type: NEPAL, 1831, Wallich 4067A (holo K000890012 digital image!).

O. japonica Naudin, Ann. Sci. Nat. Bot. Ser. 3, 14: 70. 1850. Type: JAPAN, s.loc., s.d., Textor (Blume) s.n. (holo P02441110 digital image!).

O. perrotteti DC. ex Naudin., Ann. sci. Nat. Bot. ser. 3, 14: 71. 1850. Lectotype (designated here): PHILIPPINES, Manille, Prape, s.d., Perrette s.n. (BR0000005208692 digital image!).

O. chinensis L. var. linearifolia Kuntze, Rev. Gen P1. 1: 247. 1891. Type: INDIA, West Bengal, Silligori-Terrai, s.d., C.E.O. Kuntze 6548 (holo NY00229579 digital image!)

O. chinensis L. var. parasnathensis Haines, Bot. Bihar \& Orissa 370.1922. Type: INDIA, s.loc., s.d., Clarke 34727 (holo K000890005 digital image!).

O. watanae Craib, Bull. Misc. Inform. Kew 4: 171. 1930. Type: THAILAND, Watana, 14.10.1928, Put 1909 (holo K000867933 digital image!).

O. chinensis L. var. attenuata Bakh.f., Meded Bot. Mus. Herb. Rijks Univ. Utrecht 91: 50. 1943. Type: CHINA, s.d., Elbert 987 (holo L digital image!). 
Rhexia napaulensis Ser. ex DC., Prodr. 3 : 142. 1828, pro syn.

O. ciliata Ham. in Wall., Numer List. 143: 4067 C. 1831. (pro parte), nom. nud.

O. tenuis Buch.-Ham. ex G.Don, Gen. Hist. 2: 760. 1831. (pro parte), nom. nud.

O. decora Wall., Numer. List 144, 4070. 1831. nom. nud.

O. ciliaris Buch.-Ham. ex G.Don, Gen. Hist. 2, 760. 1832. in syn. Type: Not seen.

O. glabrata Benth. in Wall., Numer. List 144. 4071. 1837. nom. nud.

O. ridleyana Hosseus., Bot. Jahrb. Syst. 45: (3) 371. 1911. nom.nud.

Fig. 25

Annual, erect, branched herbs, $c .70 \mathrm{~cm}$ tall. Stems branched, branches clothed with appressed hairs. Petioles $0.5-2.5 \mathrm{~mm}$ long; lamina ovate to lanceolate, $1.5-4.5 \times 0.8-2.4 \mathrm{~cm}, 3-5$-nerved, apex acute, base acute to rounded, both sides thinly clothed with short to long, ascending to patent hairs. Inflorescence a dense terminal cluster of 510 flowers, subtended by 1-2 pairs of leaves. Bracts ovate, minute, 1-4 × 6-8 mm, margins ciliate, appressed-hairy along the midline dorsally or sometimes glabrous. Flowers tetramerous; pedicels c. $3 \mathrm{~mm}$ long. Hypanthium campanulate, 3-7 × 2$4 \mathrm{~mm}$, with a few emergences, hairs simple; intersepalar emergences not stalked, flat, $0.3-1 \times 0.3-$ $11.5 \mathrm{~mm}$, margins ciliate, caducous; other emergences appressed, but smaller, sometimes much reduced in size. Sepals 4, triangular, 6-7 × 2-5 mm, apex acute, base obtuse, with few distinct nerves, margins ciliate, few appressed hairs dorsally, caducous, or very rarely persistent. Petals 4 , pink, ovate, 12-17 × 5-7 mm, ciliate at apex. Stamens 8; filaments 5-6.5 mm long; anthers 1.1-3.7 $\mathrm{mm}$ long beak narrowed $0.5-1.5 \mathrm{~mm}$, connective prolonged into a small collar. Ovary united with hypanthium for about half its length, anther pockets extending to base of ovary; the rim of hypanthium extending beyond the crown is glabrous or very rarely with a few bristles, crown very distinct; style 9-14 mm long.
Capsules campanulate to urceolate, 4.5-8 × 3-4 mm; seeds minute, $0.1-0.2 \mathrm{~mm}$ long, minute, curved.

\section{Key to the varieties}

1a. Intersepalar emergences caduceus var. chinensis

1b. Intersepalar emergences persistent ..... var. pusilla Osbeckia chinensis L. var. chinensis, Sp. Pl. 345. 1753

Flowering \& fruiting: September-May.

Chromosome number: $n=10$ (Meenakumari \& Kuriachan, 1990)

Habitat: This species thrives best in elevation varying from 300-500 m., commonly seen in borders of evergreen forests as well as grasslands.

Distribution: Australia, Burma, Camboclia, Celebes, China, India, Japan, Java, Laos, Lesser Sunda Islands, Malaya, Moluccas, Nepal, New Guinea, North Vietnam, Philippines, South Vietnam, Sumatra, Taiwan and Thailand.

Specimens examined: INDIA, Assam, Darrang district, Silburi range office, 30.11.1975, P.K. Hajra 65347 (ASSAM); Goalpara district, s.loc., 15.09.1937, G.K. Deka 17855 (ASSAM); Kochugaum, 27.01.1933, D. Nath 13476 (ASSAM); Golaghat district, Seelampur, 11.10.1885, C.B. Clarke 171801 (CAL); Kamrup district, Garo hills, 01.1904, J. Marten 171804 (CAL). Bihar, Begusarai district, Bomiabuna, 18.11.1938, N.L. Bor 10833 (DD); Kadalilmd, 08.01.1960, G.V. Subbarao 22927 (ASSAM). Chhattisgarh, Bastar district, North Kanjeer valley, 26.08.1959, K. Subramanyan 8633 (CAL); Dandakaranya, Orkal, 10.11.1963, D.C.S. Raju 1072 (CAL). Himachal Pradesh, Kangra district, Palampur, 25.09.1896, G.A. Gammie s.n. (DD). Jharkhand, Giridih district, Parasnath hills, 04.11.1868, s.coll. s.n. (DD); Ibid., 21.02.1923., S. Kurz.s.n. (DD); Ibid., 15.10.1982, G.N. Tribedi 718 (CAL); Ibid., s.d.,. S. Kurz. s.n, (DD); Ibid., Madhya Pradesh, Mandal district, Kanha National Park, 11.1970, R.M. Singhal s.n., (DD). Manipur, Imphal district, Chingmeirong, 03.09.1952, D.B. Deb 603 


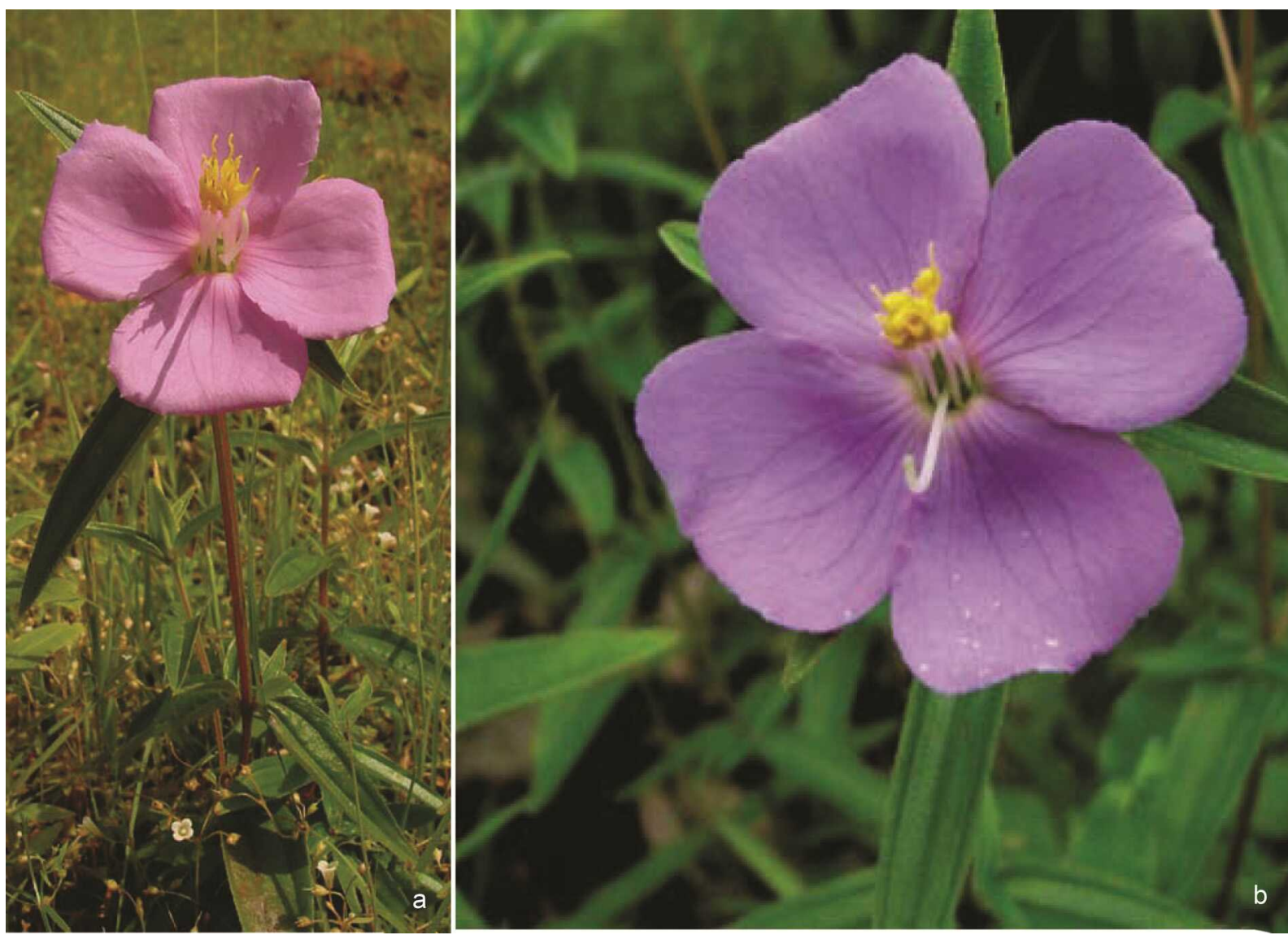

Fig. 25. Osbeckia chinensis L. var. chinensis: a. Habit; b. Flower (Images by Prasad Kumar Dash, Odisha Biodiversity Board).

(CAL); Khuduk, 18.01.1952, D.B. Deb 233 (CAL); Machahl, 04.1882, George Watt 171794, (CAL); Kakching district, Kakching, 14.11.1954, D.B. Deb 1200 (CAL); Kangpokpi, Sadar hills, 03.10.1943, N.L. Bor 17703 (DD); Konprm, 11.1907, Meebold 5907 (CAL); Shngnu, 12.09.53, D.B. Deb 2676 (CAL); Thoubal district, Wang Jing, 13.09.1954, D.B. Deb 2699 (CAL). Meghalaya, East Khasi Hills district, Barapani, 31.08.1950, G.K. Deka s.n. (ASSAM); Cherrapunji, 13.11.1910, D. Hooper 34689 (CAL); Ibid., 13.11.1910, D. Hooper 34690 (CAL); Khasia hills, 06.1876, s.coll. 274 (CAL); Ibid., 29.09.1915, H.G. Carter s.n. (CAL); Ibid., 27.09.1956, G. Panigrahi 3480 (CAL); Khasia hills, Cherra, 18.10.1932, N.K. Nandi s.n. (CAL); Mawphlang, Lawhyrgdoh, Ibid., 07.08.1885, C.B. Clarke s.n., (CAL); Ibid., 24.09.1885, C.B. Clarke s.n., (CAL); Ibid., 09.1892, Dr. King's Collector s.n. (CAL); Ibid., 05.1911, I.H. Burkill s.n. (CAL); Ibid., 26. 05 .1911, I.H. Burkill \& S.C. Banerjee s.n., (CAL); Ibid.,
29.05.1911, I.H. Burkill \& S.C. Banerjee 82 (CAL); Ibid., 07.1918, s.coll. 889 (CAL); Ibid., 09.1929, s.coll. s.n. (CAL); Ibid., 20.09.1956, G. Panigrahi 3297 (CAL); Ibid., Laitkor, way to Woodland, 28.06.1963, S.K. Kar s.n. (CAL); 29.10.1972, P.K. Hajra 51907 (CAL); Ibid., s.d., S.N. Bal 701 (CAL); Ibid., s.d., J.D. Hooker s.n., (CAL); Shillong, 03.10.1898, Dr. Prain 171766 (CAL); s.loc., 09.1878, Gustavman s.n. (ASSAM); Ibid., 12.1885, s.coll. 350 (CAL); Shillong, Nongthymmai, 20.09.1956, G Panigrahi 3297 (CAL); Upper Shillong, 14.08.1958, R.S. Rao 14034 (CAL); Ibid., 24.08.1958, R.S. Rao 14036 (CAL); West Khasi Hills district, Markasa village, 18.06.1958, G. Panigrahi 16389 (CAL); Jaintia Hills district, Raling to Garampani Road, 22.08.1968, N.P. Balakrishnan 46953 (ASSAM); Ri Bhoi district, Barapani, 13.10.1954, G.K. Deka 11452 (ASSAM); Barapani forest, 25.10.1931, Shritham Sharma 9498 (ASSAM). Mizoram, Champhai district, Zote, 10.10.1989, K.P. Singh 
94551 (ASSAM). Nagaland, Peren district, Naga hills, 10.1939, N.L. Bor 6673 (DD); Ibid., 06.1985, N.L. Bor 21000 (DD); Naga Hills, Tushar 04.09.1948, S.K. Mukerjee 3429 (CAL). Orissa, Koraput district, Deomali, 06.12.1962, G.V. Subbarao 29916 (ASSAM); Mayurbhanj district, Similipal Biosphere, Noana vicinity, 26.11.1979, s.coll. 12297 (CAL); Similipal Biosphere, Barakamara, 30.11.1979, s.coll. 12400 (CAL). Sikkim, South Sikkim district, s.loc., s.d., G. King s.n. (CAL); s.loc., 09.12.1875, J.S. Gamble 323 (DD); West Sikkim district, Gassing to Ralong river, 02.10.1862, M.D. Anderson s.n. (CAL); s.loc., 05.10.1875, C.B. Clarke s.n. (CAL); s.loc., s.d., J.D. Hooker s.n. (CAL). Tamil Nadu, Salem district, Omallur, Shevaroys temple, 19.03.1979, N. Venugopal \& Jayaselin 22374 (RHT); Yercaud, Servarayan, 29.06.1979, K.M. Matthew 23296 (RHT); Ibid., 10.01.1980, K.M. Matthew 25635 (RHT); Ibid., 16.12.1980, K.M. Matthew, Britto \& Rani 28030 (RHT). Tripura, Gomati district, Udaipur, 25.04.1965, D.B. Deb, 2885 (CAL). Uttarakhand, Dehradun district, Lachhiwala, 10.1930, A.H. Khan s.n. (DD); Tehri Garhwal, 04.10.1979, C. Supia 1056 (CAL, DD); Ibid., 09.1882, T.F. Dathu s.n. (CAL); s.loc., 30.08.1704, s.coll. s.n. (CAL); s.loc., 09.1869, Griffith s.n., (CAL); s.loc., 10.1891, J.S. Gamble 23157 (CAL, DD); Uttarkashi district, Baramgaon, Gori valley, 27.08.1900, J. Nagar 24437 (DD); Khela, Kalivally, Kumaon, 18.09.1900, Fuayal s.n. (CAL); Ibid., 18.09.1900, Juagal 24437 (CAL); Kumaon, DidihatNarayan nagar, 12.10.1967, Arora 36491 (BSD); Sarayu valley, 28.09.1881, Dutha, 1607 (DD); Ibid., 19.08.1986, Dutha 5575 (DD). West Bengal, Darjeeling district, s.loc., 10.03.1995, Chandra Panday 21597 (CAL); s.loc., s.d., Kurz 171844 (CAL).

Notes: Osbeckia chinensis is the wide spread species of the genus in Asia. It is very variable, and too many species have been published based on specimens falling within its variation. For example, two entities within $O$. chinensis have been recognized such as var. attenuata Bakh. and var. parasnathensis. Hansen (1977) treated these varieties as conspecific to O. chinensis. He also described var. procumbens from northern Thailand owing to its creeping habit. Hansen (l.c.) admitted var. pusilla and var. procumbens as varieties in his Asiatic Revision of Osbeckia.

Osbeckia chinensis L. var. pusilla (Zoll.) Triana, Trans. Linn. Soc., London 28: 53. 1872. O. pusilla Zoll. \& Moritzi, Natuur. Geneesk. Arch. Ned.Indie 2: 586. 1845, non De Wild. 1921. Lectotype (designated by Hansen, 1977): INDONESIA, Java, Zollinger 2225 (P02441165 digital image!).

O. truncata D.Don ex Wight \& Arn. var. strigosa Kuntze, Rev. Gen. Pl. 1: 248. 1891. Type: INDIA, s.loc., s.d., Kuntze 6219 (holo NY229590 digital image!).

O. boissieuana Guillaumin, Notul. Syst. (Paris) 2: 302. 1912; \& in Bull. Soc. Bot. France 365. 402.1913. Lectotype (designated here): VIETNAM, Cochinchine, 1862, Thorel 914 (BM000944330 digital image!).

O. parva E.T.Geddes, Bull. Misc. Inform., Kew 2: 70. 1928. Type: THAILAND, $3000 \mathrm{ft}, 06.10 .1912$, Kerr 2719 (holo K000867932 image!).

Perennial, erect, branched herbs $c .70 \mathrm{~cm}$ tall. Stems branched, branches clothed with appressed hairs. Petioles 0.5-2.5 mm long, Lamina lanceolate, usually more than 3 times as long as broad, 2-6 $\times$ 1-2.5 cm, 3-5-nerved, apex acute, base acute to rounded, both sides thinly clothed with short to long, ascending to patent hairs. Inflorescence a dense terminal cluster of 5-10 flowers subtended by 1-2 pairs of leaves. Flowers tetramerous; bracts minute, ovate, 1-3 × 3-4 mm, margins ciliate and appressed-hairy along the midline dorsally, sometimes glabrous; pedicels c. $3 \mathrm{~mm}$ long. Hypanthium campanulate, 4-6 × 3-4 mm mm, with a few emergences, simple hairs, intersepalar emergences not stalked, flat, 0.3-1 ×0.3-11.5 mm, margins ciliate, persistent; other emergences, appressed, but smaller, sometimes much reduced in size. Sepals 4, triangular, 1-4 mm long, apex acute, base obtuse, margins ciliate, with a few 
appressed hairs dorsally, caducous, or very rarely persistent. Petals 4, pink, ovate, 7-10 × 5-7 mm, ciliate at apex. Stamens 8; filaments 2-4 mm long; anthers 2-4.5 $\mathrm{mm}$ long beak narrowed, 2-2.5 mm, connective prolonged into a small collar. Ovary united with hypanthium for about half its length, anther pockets extending to base of ovary; free part glabrous or very rarely with a few bristles, crown very distinct; style 5-12 $\mathrm{mm}$ long. Capsules campanulate, 4-6 × 3-4 mm; seeds $0.1-0.2 \mathrm{~mm}$ long, minute, curved.

Flowering \& fruiting: September-May.

Chromosome number: $n=10$ (Meenakumari \& Kuriachan, 1990).

Habitat: Species thrives in massive population at elevation varying from 300-1500 m., commonly seen in borders of evergreen forests as well as grasslands.

Distribution: Burma, Cambodia, Java, India, Laos, Lesser Sunda Islands, South Vietnam and Thailand.

Specimen examined: INDIA, Andaman \& Nicobar Islands, North Andaman, 01.12.1976, N.P. Balakrishnan \& N.G. Nair 4772 (PBL).

Notes: Osbeckia chinensis var. pusilla often grows in grasslands. It has been collected at elevation up to $1570 \mathrm{~m}$.

Osbeckia zeylanica L.f., Suppl. Sp. Pl. 215. 1782; C.B.Clarke in Hook.f., Fl. Brit. India 2: 516. 1879; Gamble, Fl. Pres. Madras 1: 494. 1919; C.Hansen, Ginkgoana 4: 43. 1977; J.L.Ellis, Fl. Nallamalais 186. 1987; Ramach. \& V.J.Nair, Fl. Cannanore district 188. 1988; Antony, Syst. Stud. Fl. Kottayam district 168. 1989; Sasidh., Fl. Periyar Tiger Reserve 145. 1998; Sasidh., Fl. Chinnar W.L.S. 132. 1999; B.D.Sharma, Karthik. \& N.P.Singh, Fl. Maharashtra, Dicot. 28. 2000; J.M.Pallithanam \& K.M.Matthew, Fl. Sirumalai Hills, South India 107. 2001; Mohanan \& Sivad., Fl. Agasthyamala 281. 2002; Pull., D.M.Rao \& K.S.Ramamurthy, Fl. Eastern Ghats 60. 2002. Type: s.loc., s.d., s.coll. (holo LINN S09-32833 digital image!).
O. debilis Naudin, Ann. Sci. Nat. Bot. ser. 3, 14: 65. 1850. Type: s.loc, s.d., s.coll. s.n. (holo P02441158 digital image!).

O. serialis Naudin, Ann. Sci. Nat. Bot. ser. 3, 14: 67. 1850. Type: INDIA, Tamil Nadu, Courtallum, Vendalore, 1836, Wight 1143 (holo P02441159 digital image!).

O. zeylanica L.f. var. non-rostrata Haines, Bot. Bihar \& Orissa 369. 1922. Lectotype (designated here): s.loc., s. d., Haines 4787 (K000890017 digital image!).

Echinophora maderaspatana Pluk. ex Bakh.f., Meded. Bot. Mus. Herb. Rijks Univ. Utrecht 91: 48. 1943, pro syn.

Fig. 26

Annual, erect herbs, 4-45 cm tall. Stems quadrangular, hispid, sparsely appressed-hairy, branched or unbranched. Petioles 1-4 mm long; lamina ovate, $1.5-4 \times 0.6-1.5 \mathrm{~cm}$, cuneate at base, acute at apex, densely hairy on both sides, hairs ascending, thin. Inflorescence a terminal cluster of 5-7 flowers or solitary, subtended by a pair of leaves. Flowers tetramerous; pedicels c. $3 \mathrm{~mm}$ long; bracts triangular, green, $1.5-3 \times 1-2 \mathrm{~mm}$, margins ciliate. Hypanthium campanulate, 3-5 × 1.5-2.5 $\mathrm{mm}$, thinly clothed with patent emergences and hairs; intersepalar emergences 1-2 mm long, stalk terete, with patent hairs, head with stellate hairs (1$2 \mathrm{~mm}$ long), persistent; other emergences similar, decreasing in size towards base of hypanthium. Sepals 4, triangular, 2.5-5.5 × 1-2 $\mathrm{mm}$, prominently one nerved, margins ciliate, stellate hairs at apex, few patent hairs on nerves dorsally, persistent in fruit. Petals 4,obovate, pink to purple, 8-12 × 3-10 mm, ciliate at apex. Stamens 8; filaments 3-5 mm long; anthers twisted, 2.5-6 mm long, yellow, attenuate into a narrow twisted beak of c. $1 \mathrm{~mm}$, connective prolonged into a widened collar. Ovary united with hypanthium for about half its length; anther pockets extending to the base of the ovary; crown absent; style 7-12 mm long; stigma slightly widened. Capsules campanulate, $3-$ $5 \times 2-3 \mathrm{~mm}$, ribbed, exceeds ovary; seeds minute 0.1-0.2 mm long, numerous, curved. 


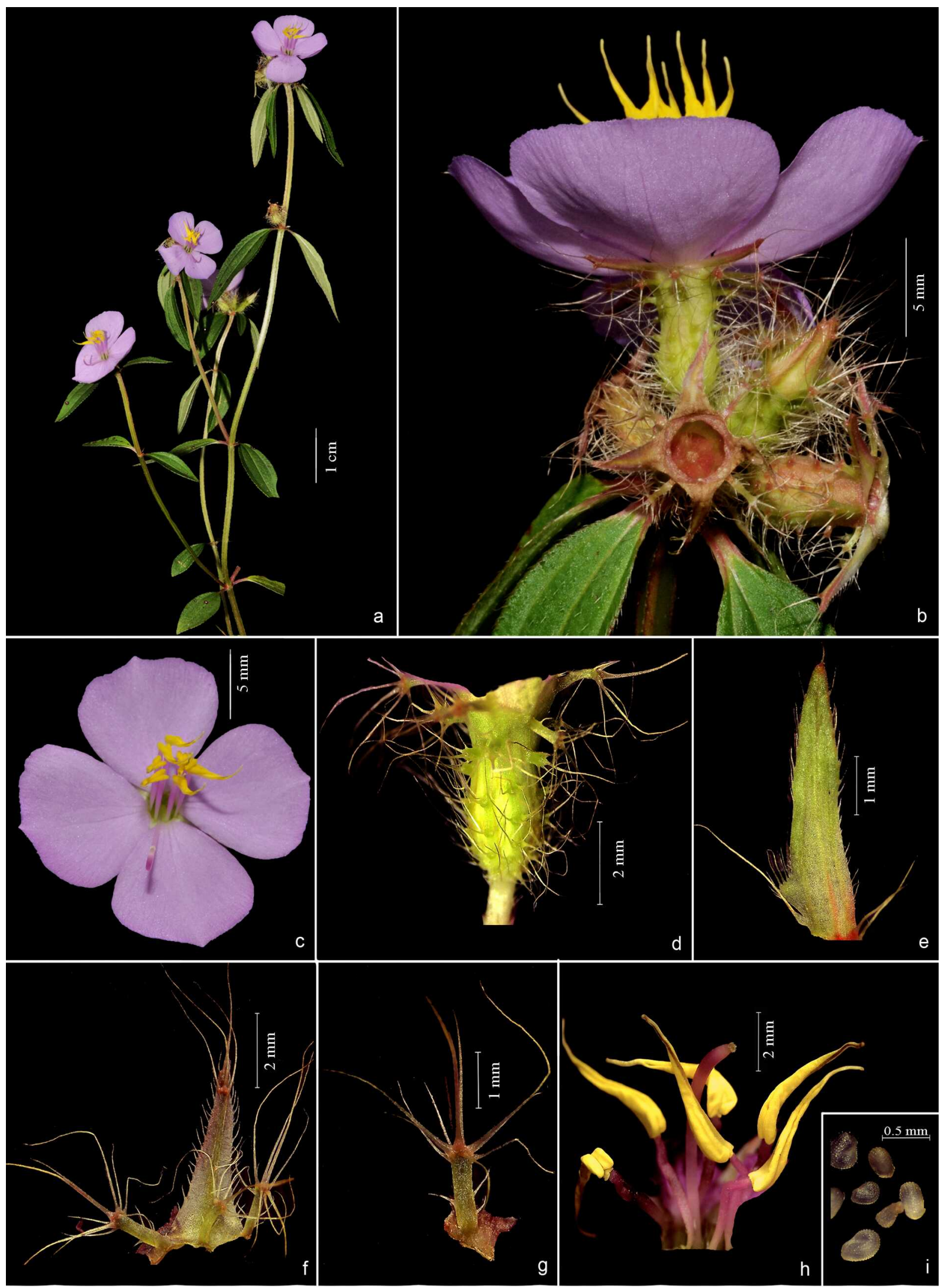

Fig. 26. Osbeckia zeylanica L.f.: a. Habit; b. Inflorescence; c. Flower; d. Hypanthium; e. Bract; f. Calyx lobe \& intersepalar emergences; g. Intersepalar emergence; h. Stamens; i. Seeds. 
Flowering \& fruiting: November-March.

Chromosome number: $n=10$ (Meenakumari \& Kuriachan, 1990)

Habitat: Occurs abundantly in rock crevices in deciduous forests at an elevation above $500 \mathrm{~m}$.

Distribution: India and Sri Lanka.

Specimens examined: INDIA, Andhra Pradesh, Chittoor district, Chandragiri, 22.02.1914, s.coll. 9979 (MH); Chrudregira fort, 04.02.1089, Ranga 2267 (MH); Gyanayagundlu, 28.12.1975, G.V. Subbarao 46818(CAL, MH); Mamandar, 11.03.1918, C.E.C. Fischer 171873 (CAL); Sunnipenta Nallamd, J.L. Ellis 23774 (CAL); Cuddapah district, Balapalle, 23.02.1963, J.L. Ellis 15765 (CAL, MH); East Godavari district, Addateegala, 26.09.1980, G.V. Subbarao 68590 (MH); Karimnagar district, Aklaspur, 30.12.1964, G.V.Subbarao 22485 (CAL, MH); Nellore district, Tada, 03.03.1913, s.coll. 21242 (MH); Tada lake vicinity, 26.02.1971, Sastry 9748 (CAL). Karnataka, Chickmagalur district, Kadur, 14.01.1979, K.R. Keshava Murthy, B.R. Ramesh \& P. Prakesh 5685 (CAL). Kerala, Kannur district, Iritty, 11.11.1978, V.S. Ramachandran 58660 (CAL, $\mathrm{MH})$; Idukki district, Erattayar, 13.10.1982, V.S. Ramachandran \& Mohanan 74611 (MH); Kozhikanam, 11.11.1975, Vivekananthan 46629 (MH); Travancore, 27.11.1913, M. Rama Rao 171872 (CAL). Madhya Pradesh, Ganjam, 01.1890, J.S. Gamble 21607 (CAL); Indravati Tiger reserve-Bijapur Ghati, 11.10.1987, Anand Kumar 16150 (CAL); s.loc., s.d., s.coll. 21235 (MH). Orissa, Puri district, Puri coast, 02.12.1965, T.A. Rao 5698 (CAL); Orissa coast, 09.03.1909, H.H. Haines 171725 (CAL); Orissa-Konark coast, 04.12.1965, T.A. Rao 5953(CAL). Tamil Nadu, Coimbatore district, Atakathi, Anaimali hills, 26.12.1911, C.E.C. Fischer 771881 (CAL); Dharmapuri district, Harur, Chitteri hills, 12.01.1979, Venugopal 20851 (RHT); Kalachipodi Chitteri, 27.12.1990, Ravishankar $95555(\mathrm{MH})$; Dindigul district, Kodaikanal, 20.02.1986, K.M. Matthew 44218 (RHT); Ibid., 02.05.1988, K.M. Matthew 52873 (RHT); Madras,
02.1889, J.S. Gamble 20390 (CAL); Situmalai, 09.10.1959, Pallithanam 5172 (RHT); Kanchipuram district, Chingleput, M.C.C. campus, 05.02.1986, Narasimhan 954 (MH); Chingleput, Tirupporur, 01.02.1976, A.N Henry 47145 (CAL); Puzhuthivakkam, 31.01.2000, K.M. Matthew E Jayanthi 70982 (RHT); Tambaram, M.C.C Campus, 05.01.2001, Livingston 72077 (RHT); Thenpattinam, 06.03.2000, Augustine 61955 (RHT); Krishnagiri district, Denkanikottai, 14.11.1979, K.M. Matthew 24576 (RHT); Pudukkottai district, Kulathur, Bommadimalai, 09,03.1984, K.M. Matthew \& Britto 29676 (RHT); Kulathur, Narathamalai, Ibid., 09,01.1969, K.M. Matthew 8268 (RHT); Ibid., 20,10.1970, K.M. Matthew 12857 (RHT); Ibid., 15,11.1975, K.M. Matthew 15073 (RHT); Ibid., s.d., Arulappan. s.n. (MH); Ibid., 10.01.1971, K.M. Matthew 12981 (RHT); Ibid., 07.02.1980, Diraviam 26354 (RHT); Ibid., 16.12.1982, K.M. Matthew \& Rani 29336 (RHT); Sathyamangalam, 23.12.1978, s.coll., 16092 (RHT); Tirunelveli district, Agasthiyar falls, 12.02.2015, Prashob \& Sibichen 5596 (DEV); Ambasamudram, Kannikketti, 02.01.1978, K.M. Matthew 15826(RHT); Kalakkad, 22.01.1979, K.M. Matthew 16232 (RHT); Ibid., 12.02.2015, Prashob \& Sibichen 5594 (DEV); Kannikketti, 20.02.1913, Ramaswami 39396 (CAL); Mundanthurai, 19.02.1913, M.S Ramaswami, 39340 (CAL); Ibid., 22.02.1960, K.M. Sebastine 9909 (CAL); Papanasam, 12.02.2015, Prashob \& Sibichen 5595 (DEV); Sivanapuri, 09.02.1916, C.E.C. Fischer 3876 (CAL); Tanimapatam, 02.1889, J.S. Gamble 20390 (CAL); Viluppuram district, Kallakurichi, 15.01.1979, Venugopal 21045 (RHT); Viluppuram, Tonnadu malai, 10.01.1999, K.M. Matthew \& Augustine 71081 (RHT); Ulundurpet, South Arcot, 30.12.1981, K.M. Matthew, Britto \& Rani 28741 (RHT); Ibid., 01.02.1980, K.M. Matthew, Britto \& Rani 26264 (RHT).

Notes: Osbeckia zeylanica is a distinct species differing from all other annual species by the twisted anthers, which gradually narrowing into a beak with a small bend near the tip pointing the pore towards the back. 
The consistent, but unfounded confusion of O. zeylanica with O. chinensis has been solved by Hansen (1972).

\section{Acknowledgements}

Authors are grateful to the Principal, and the Head, P.G. and Research Department of Botany, St. Joseph's College (Autonomous), Devagiri, Kozhikode for facilities; Dr. Santhosh Nampy, Dr. A.K. Pradeep (University of Calicut) and Dr. Manudev K.M. (St. Joseph's College, Kozhikode), for various helps. Authors wish to thank the Directors/In-charges/ curators of BSD, BSI, CAL,CALI, DD, JCB, KFRI, $\mathrm{MH}$, RHT, DEV, TBGT for herbarium consultation, and the curators of BM, BR, B-W, E, C, G, G-DC, HAL, JE, K, K-W, L, LINN, P, S, U, US, W, WA and WU for providing the digital images of types. Authors are also grateful to the Kerala State Council for Science, Technology and Environment (KSCSTE) and Rashtriya Uchchatar Shiksha Abhiyan (RUSA) for financial assistance.

\section{Literature Cited}

BEDDOME R.H. 1864. Contribution to the botany of southern India. Madras Journal of Literature and Science 1(3): 45.

BLUME C.L. 1849. Museum Botanicum Lugduno-Batavum sive stirpium Exoticarum, Novarum vel Minus Cognitarum ex Vivis aut Siccis Brevis Expositio et Descriptio. E.J. Brill, Leiden.

BRIDSON D. \& L. FORMAN 1992. The Herbarium Handbook. Royal Botanic Gardens, Kew.

CLARKE C.B. 1879. Melastomataceae In: HOOKER J.D. (ed.) Flora of British India. Volume 2. L. Reev \& Co. Ltd., London. pp. 513-522.

COGNIAUX C.A. 1891. Melastomaceae In: A. \& C. DE CANDOLLE (eds.), Monographiae Phanerogamarum, Volume 7, G. Masson, Paris. pp. 313-335.

FAVARGER C. 1962. Nouvelles recherches cytologiques sur les Melastomatacees. Ber Bericht der Schweizerischen Botanischen Gesellschaft 72: 290-305.

GIRI G.S. 1983. A new species of Osbeckia L. (Melastomataceae) from India. Journal of Economic and Taxonomic Botany 4(2): 609-611.

GIRI G.S. \& M.P. NAYAR 1983. New taxa of Osbeckia L. with a note on O. lineolata Gamble. Bulletin of the Botanical Survey of India 25(1-4): 241-245.
GIRI G.S. \& M.P. NAYAR 1984. A new species of Osbeckia L. (Melastomataceae) from Kerala (India). Journal of Bombay Natural History Society 81(2): 434-436.

GIRI, G.S. \& M.P. NAYAR 1986. A new species of Osbeckia (Melastomataceae) from southern India. Kew Bulletin 41(2): 429-431.

HANSEN C. 1972. Osbeckia chinensis L. and O. zeylanica L.f. (Melastomataceae). Taxon 21: 653-657.

HANSEN C. 1977. The Asiatic species of Osbeckia (Melastomataceae). Ginkgoana 4: 1-150.

IUCN 2001. IUCN Red List Categories and Criteria: Version 3.1. IUCN Species Survival Commission IUCN, Gland and Cambridge.

IUCN 2019. The IUCN Red List of Threatened Species. http:// www.iucnredlist.org (Accessed 25.12.2019).

KOTTAIMUTHU R. \& G. GNANASEKARAN 2015. Validation of the name Osbeckia tirunelvelica (Melastomataceae). Phytotaxa 231(3): 300.

LINNAEUS C. 1753. Species Plantarum. Volume 1. Laurentii Salvii, Stockholm.

LINNAEUS C. VON 1781. Supplementum Plantarum Systematis Vegetabilium Editionis Decimae Tertiae, Generum Plantarum Editiones Sextae, et Specierum Plantarum Editionis Secundae. Impensis Orphanotrophei, Brunsvigae. p. 468.

MABBERLEY D.J. 2017. Mabberley's Plant Book-A Portable Dictionary of Plants, their classification and uses. Fourth Edition. Cambridge University Press, Cambridge. p. 1040.

MAITY D. 2003. Taxonomic states of Osbeckia nutans Wall. ex C.B. Clarke (Melastomataceae). Journal of Economic and Taxonomic Botany 27: 1235-1237.

MANICKAM V.S. \& C. MURUGAN 2002. Osbeckia tirunelvelica, a new species of Melastomataceae from Western Ghats, India. Journal of Economic and Taxonomic Botany 25(3): 626-628.

MEENAKUMARI R. \& P. KURIACHAN 1990. SOCGI plant chromosome number reports X. Journal of Cytology and Genetics 25: 321-322.

MEHRA P.N. \& P.K. KHOSLA 1969. IOPB chromosome number reports XX. Taxon 18: 213-221.

NAUDIN C. 1850. Melastomacearumque in Musieo Parisiensi continentur Monographicae Descriptionis et Secundum affinitates distributionis. Tentamen. Annales des Sciences Naturelles Botanique 14: 53-76.

NAYAR M.P. \& G.S. GIRI 1984. Osbeckia arunkumarensis Sp. Nov. from eastern India. Journal of Bombay Natural History Society 81(2): 436-439.

PRASHOB P. MANUDEV K.M. SIBICHEN M. THOMAS \& SANTHOSH NAMPY 2018. Osbeckia saddlepeakensis (Melastomataceae): a new species from 
Andaman and Nicobar Islands, India. Phytotaxa 344(2): 185-190.

ROXBURGH W. 1832. Flora Indica or descriptions of Indian Plants, Volume 1. W. Thacker and Co., Kolkata.

SUBRAMANYAM K. 1946. Further contribution to the embryology of the genus Osbeckia L. Half Yearly Journal of Mysore University 7(1): 1-11.

THIERS B.M. 2018. Index Herbariorum: A global directory of public herbaria and associated staff. New York Botanical Garden's virtual Herbarium. http://sweetgum.nybg.org/ih/

TRIANA J.J. 1871. Les Melastomace'es. Transactions of the Linnaean Society, London 28: 1-188.

TRIMEN H. 1894. A hand-book to the flora of Ceylon: containing descriptions of all the species of flowering plants indigenous to the island, and notes on their history, distribution, and uses: with an atlas of plates illustrating some of the more interesting species. Volume 2. Dulau \& Co. London. p. 196.

TURLAND N.J., WIERSEMA J.H., BARRIE, F.R., GREUTER W., HAWKSWORTH D.L., HERENDEEN P.S., KNAPP S., KUSBER W.-H., LI
D.-Z., MARHOLD K., MAY T.W., MCNEILL J., MONRO, A.M., PRADO J., PRICE M.J. \& G.F. SMITH (EDS.) 2018. International Code of Nomenclature for algae, fungi, and plants (Shenzhen Code) adopted by the Nineteenth International Botanical Congress Shenzhen, China, July 2017. Regnum Vegetabile 159. Glashütten: Koeltz Botanical Books. https://doi.org/10.12705/Code. 2018

VAHL M. 1807. Eclogae Americanae seu descriptiones plantarum praesertim Americae meridionalis, nondum cognitarum. Volume 3. Impensis Auctoris, Havniae.

VERANSO-LIBALAH M.C., STONE, R.D., FONGOD, A.G.N., COUVREUR, T.L.P. \& G. KADEREIT 2017. Phylogeny and systematics of African Melastomateae (Melastomataceae). Taxon 66: 584-614.

VOGEL E.F. DE 1987. Manual of Herbarium Taxonomy: Theory and Practice. Rijks Herbarium, Leiden.

WALLICH N. 1831. Numerical list of dried specimens of plants in the Museum of the Honl: East India Company or Description and figure of selected number of unpublished East Indian Plants. Treuttel and Wartz: Treuttel Junr. and Richter, London. pp. 143-144. 